\title{
Stereochemistry of Nucleophilic Substitution Reactions Depending Upon Substituent: Evidence for Electrostatic Stabilization of Pseudoaxial Conformers of Oxocarbenium Ions by Heteroatom Substituents
}

Leticia Ayala, Claudia G. Lucero, Jan Antoinette C. Romero, Sarah A. Tabacco, and Keith A. Woerpel*

Department of Chemistry, University of California, Irvine, California 92697-2025

I. General Information Supplementary Information

II. Synthesis of Acetates

S-1

A. Synthesis of 4-(tert-Butyldiphenylsilyloxy)tetrahydropyran-1-acetate (14a) S-2

B. Synthesis of 4-Methanesulfoxytetrahydropyran-1-acetate (14b)

$\mathrm{S}-5$

C. Synthesis of 4-Benzoyloxytetrahydropyran-1-acetate (14c)

$\mathrm{S}-7$

D. Synthesis of 4-(p-Anisoyloxy)tetrahydropyran-1-acetate (14d)

S-10

E. Synthesis of 4-( $p$-Nitrobenzoyloxy)tetrahydropyran-1-acetate (14e)

$\mathrm{S}-13$

F. Synthesis of 4-Nitrotetrahydropyran-1-acetate (14f)

G. Synthesis of 4-Azidotetrahydropyran-1-acetate (14g)

S-16

S-18

H. Synthesis of 4-Fluorotetrahydropyran-1-acetate (21a)

$\mathrm{S}-21$

I. Synthesis of 4-Chlorotetrahydropyran-1-acetate (21b)

$\mathrm{S}-28$

J. Synthesis of 4-Bromotetrahydropyran-1-acetate (21c)

S-29

K. Synthesis of 4-Iodotetrahydropyran-1-acetate (21d)

S-31

L. Synthesis of 2-Isopropyltetrahydropyran-1-acetate (32c)

$\mathrm{S}-34$

M. Synthesis of 2-tert-Butyltetrahydropyran-1-acetate (32d)

S-37

N. Synthesis of $(4 S, 5 R)$-2-Acetoxy-5-benzyloxy-4-methyltetrahydropyran -1-acetate (43)

III. Allylation Reactions

IV. Nucleophilic addition of Diethylzinc

V. Nucleophilic addition of 1-Phenyl-1-(trimethylsilyloxy)ethylene S-62

VI. Stereochemical Proofs

A. $\quad{ }^{1} \mathrm{H}$ NMR Coupling Constant Data $\quad$ S-63

$\begin{array}{ll}\text { B. DPFSG-NOE Data } & \text { S-68 }\end{array}$

VII. Bibliography

S-68

VIII. GC Analysis of Isomer Ratios and ${ }^{1} \mathrm{H}$ NMR Spectra of Selected Compounds $\quad$ S-74

Note: Details for experiments that were reported in the Communication of this work (J. A. C. Romero, S. A. Tabacco, K. A. Woerpel, J. Am. Chem. Soc. 2000, 122, 168-169) have already been published on the Web as Supporting Information. Consequently, that information was not duplicated in the Supporting Information for this Article.

\section{General Information}

${ }^{1} \mathrm{H}$ NMR and ${ }^{13} \mathrm{C}$ NMR spectra were recorded at ambient temperature at $500 \mathrm{MHz}$ and $125 \mathrm{MHz}$, respectively, using a Nicolet Omega 500 or a GN 500 spectrometer. All chemical shifts are reported in ppm relative to tetramethylsilane on the $\delta$ scale, multiplicity $(\mathrm{br}=$ broad, $\mathrm{s}=$ singlet, $\mathrm{d}=$ doublet, $\mathrm{t}=$ triplet, $\mathrm{q}=$ quartet, quint = quintet, and $\mathrm{m}=$ multiplet , , coupling constants 
in $\mathrm{Hz}$, and integration. The ${ }^{19} \mathrm{~F}$ NMR spectra were recorded in ppm downfield from internal standard $\mathrm{CFCl}_{3}$ in $\mathrm{CDCl}_{3}$ at $376 \mathrm{MHz}$ using a Bruker DRX 400. Infrared (IR) spectra were obtained using a MIDAC Prospect FT-IR spectrometer. High resolution mass spectra were acquired on a VG Analytical 7070E or Fisons Autospec spectrometer. Elemental analyses were performed by Atlantic Microlab, Atlanta, GA. Analytical gas-liquid chromatography (GC) analyses were performed on a Hewlett Packard 5890 Level 4 Chromatograph, equipped with a split-mode injection system and a flame ionization detector. Fused silica capillary column (30 m $\times 0.32 \mathrm{~mm}$ ) wall coated with DB-1 (J \& W Scientific) was used with helium as the carrier gas (16 psi column head pressure). Liquid chromatography was performed using force flow (flash chromatography) with the indicated solvent on $60 \AA$ SiliTech silica gel $\left(\mathrm{SiO}_{2}\right)$. All reactions were carried out under nitrogen atmosphere in flame-dried glassware using standard syringe/septa techniques. Unless otherwise stated, all reagents were used unpurified from the supplier. $\mathrm{CH}_{2} \mathrm{Cl}_{2}$ and THF were dried non-pyrophorically as in the method of Grubbs. ${ }^{1}$ All other solvents were dried and distilled. $\mathrm{NaH}$ was washed with hexanes and dried under reduced pressure.

\section{Synthesis of Acetates}

\section{IIA. Synthesis of 4-(tert-Butyldiphenylsilyloxy)tetrahydropyran-1-acetate (14a)}

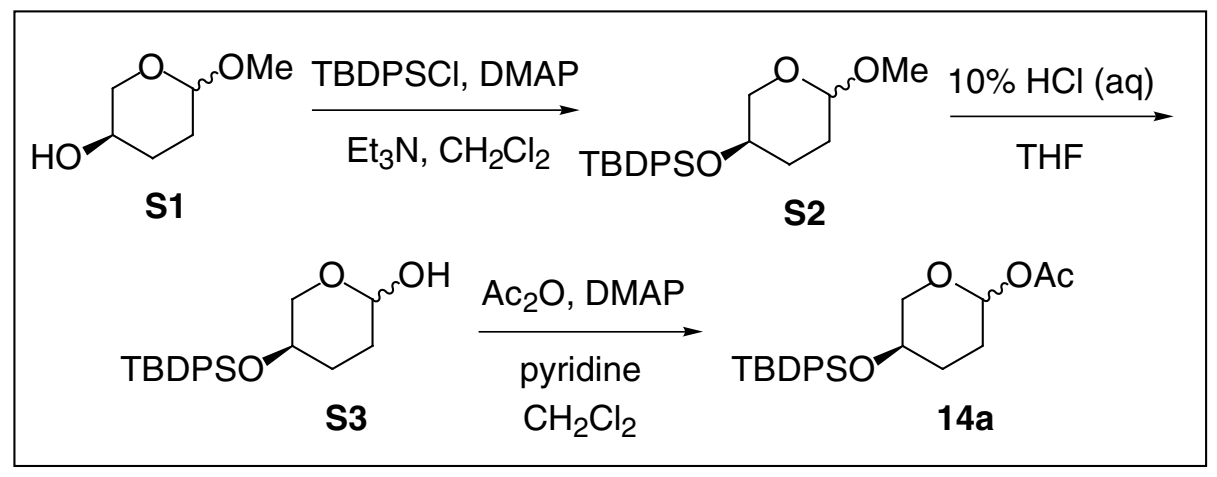<smiles>CO[C@H]1CC[C@@H](O[R5](C)(C)C)CO1</smiles> 
4-(tert-Butyldiphenylsilyloxy)-1-methoxytetrahydropyran (S2): Triethylamine $(8.39 \mathrm{~mL}$, $60.2 \mathrm{mmol})$ and 4-dimethylaminopyridine (0.886 $\mathrm{g}, 7.22 \mathrm{mmol})$ were added to a cooled $\left(0{ }^{\circ} \mathrm{C}\right)$ solution of 4-hydroxy-1-methoxytetrahydropyran in $\mathrm{CH}_{2} \mathrm{Cl}_{2}(12 \mathrm{~mL}){ }^{2}$ followed by slow addition of $t$-butylchlorodiphenylsilane $(8.14 \mathrm{~mL}, 31.3 \mathrm{mmol})$. The mixture was warmed slowly to room temperature and stirred for $15 \mathrm{~h}$ before addition of water $(100 \mathrm{~mL})$ to dissolve the white precipitate. The layers were separated, and the aqueous layer was extracted with $\mathrm{Et}_{2} \mathrm{O}(3 \times 50$ $\mathrm{mL})$. The combined organic layers were washed with $1 \mathrm{~N} \mathrm{HCl}(30 \mathrm{~mL})$, saturated aqueous $\mathrm{NaHCO}_{3}(30 \mathrm{~mL})$, and brine $(30 \mathrm{~mL})$, dried over $\mathrm{MgSO}_{4}$, filtered and concentrated in vacuo. The resulting oil was purified by flash chromatography (9:1 to 4:1 EtOAc/hexanes) to afford the product (as a 3.3:1 mixture of anomers) as a colorless oil $(7.41 \mathrm{~g}, 83 \%):{ }^{3}{ }^{1} \mathrm{H}$ NMR $(500 \mathrm{MHz}$, $\left.\mathrm{CDCl}_{3}\right) \delta 7.68-7.64(\mathrm{~m}, 17.2 \mathrm{H}), 7.44-7.41(\mathrm{~m}, 8.6 \mathrm{H}), 7.38-7.35(\mathrm{~m}, 17.2 \mathrm{H}), 4.54(\mathrm{dd}, J=4.8$, 2.9, 3.3H), $4.46(\mathrm{dd}, J=3.1,2.0,1 \mathrm{H}), 3.77-3.67(\mathrm{~m}, 7.6 \mathrm{H}), 3.52(\mathrm{t}, J=10.1,1 \mathrm{H}), 3.42(\mathrm{ddd}, J=$ $10.7,4.8,1.9,1 \mathrm{H}), 3.37$ (s, 9.9H), 3.34 (s, 3H), 3.32 (ddd, $J=10.3,5.2,1.2,3.3 \mathrm{H}), 2.08$ (ddd, $J$ $=13.4,9.9,4.6,3.3,3.3 \mathrm{H}), 1.88(\mathrm{ddt}, J=13.2,8.8,4.4,3.3 \mathrm{H}), 1.81(\mathrm{~m}, 1 \mathrm{H}), 1.76-1.67(\mathrm{~m}, 2 \mathrm{H})$, $1.58(\mathrm{~m}, 3.3 \mathrm{H}), 1.51(\mathrm{dt}, J=13.4,3.8,1 \mathrm{H}), 1.44(\mathrm{ddt}, J=12.1,7.8,4.7,3.3 \mathrm{H}), 1.07$ (s, 29.7H), $1.05(\mathrm{~s}, 9 \mathrm{H}) ;{ }^{13} \mathrm{C}$ NMR (125 MHz, $\left.\mathrm{CDCl}_{3}\right) \delta 135.9,135.7,134.8,134.13,134.09,134.0$, 129.69, 129.65, 129.4, 127.8, 127.6, 127.5, 127.4, 99.9, 97.2, 67.1, 66.51, 66.46, 64.4, 55.3, 54.6, 28.6, 28.0, 27.6, 27.0, 26.9, 26.7, 19.2, 19.1; IR (thin film) 3071, 2932, 1428, 1216, 1109, 823, $703 \mathrm{~cm}^{-1}$; HRMS (CI/isobutane) $m / z$ calcd for $\mathrm{C}_{21} \mathrm{H}_{27} \mathrm{O}_{2} \mathrm{Si}\left(\mathrm{M}-\mathrm{CH}_{3} \mathrm{O}\right)^{+}$339.1780, found 339.1771. Anal. Calcd for $\mathrm{C}_{22} \mathrm{H}_{30} \mathrm{O}_{3} \mathrm{Si}$ : C, 71.31; H, 8.16. Found: C, 71.15; H, 8.28.

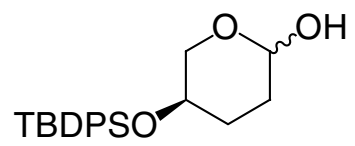

S3

4-(tert-Butyldiphenylsilyloxy)tetrahydropyran-1-ol (S3): An aqueous (10\%) solution of $\mathrm{HCl}(10 \mathrm{~mL})$ was added slowly to a solution of 4-(tert-butyldiphenylsilyloxy)-1- 
methoxytetrahydropyran in THF $(20 \mathrm{~mL})$. After $36 \mathrm{~h}$, the mixture was neutralized with saturated aqueous $\mathrm{NaHCO}_{3}$. The THF was removed in vacuo, and the remaining aqueous layer was extracted with $\mathrm{Et}_{2} \mathrm{O}(3 \times 25 \mathrm{~mL})$, dried over $\mathrm{MgSO}_{4}$, filtered, and concentrated in vacuo. The resulting oil was purified by flash chromatography (1:9 to 2:3 EtOAc/hexanes) to afford the product (as a 1:1 mixture of anomers) as a colorless oil $(0.898 \mathrm{~g}, 96 \%):{ }^{3}{ }^{1} \mathrm{H}$ NMR (500 MHz, $\left.\mathrm{CDCl}_{3}\right) \delta 7.68-7.64(\mathrm{~m}, 8 \mathrm{H}), 7.46-7.44(\mathrm{~m}, 4 \mathrm{H}), 7.43-7.35(\mathrm{~m}, 8 \mathrm{H}), 4.97(\mathrm{dd}, J=7.2,3.5,1 \mathrm{H})$, 4.91 (ddd, $J=7.1,4.8,2.3,1 \mathrm{H}), 3.83$ (ddd, $J=11.3,3.7,1.7,1 \mathrm{H}), 3.79-3.68$ (m, 3H), 3.45 (ddd, $J=10.8,3.8,1.6,1 \mathrm{H}), 3.34(\mathrm{dd}, J=11.4,7.1,1 \mathrm{H}), 2.56(\mathrm{~d}, J=5.0,1 \mathrm{H}), 2.46(\mathrm{dd}, J=1.4,4.2$, 1H), $2.02(\mathrm{~m}, 1 \mathrm{H}), 1.99-1.77(\mathrm{~m}, 3 \mathrm{H}), 1.66(\mathrm{~m}, 1 \mathrm{H}), 1.61-1.52(\mathrm{~m}, 2 \mathrm{H}), 1.38(\mathrm{~m}, 1 \mathrm{H}), 1.06(\mathrm{~s}$, $18 \mathrm{H}) ;{ }^{13} \mathrm{C}$ NMR $\left(125 \mathrm{MHz}, \mathrm{CDCl}_{3}\right) \delta 135.71,135.68,135.65,134.1,134.01,133.96,129.73$, 129.70, 129.67, 127.8, 127.64, 127.62, 127.59, 94.4, 91.8, 68.1, 66.7, 66.5, 65.8, 29.0, 28.9, 28.7, 27.8, 27.0, 26.9, 19.2, 19.1; IR (thin film) 3390, 2931, 2360, 1427, 1111, 822, $701 \mathrm{~cm}^{-1}$; HRMS (CI/isobutane) $m / z$ calcd for $\mathrm{C}_{21} \mathrm{H}_{28} \mathrm{O}_{3} \mathrm{SiNa}(\mathrm{M}+\mathrm{Na})^{+}$379.1705, found 379.1708. Anal. Calcd for $\mathrm{C}_{21} \mathrm{H}_{28} \mathrm{O}_{3} \mathrm{Si}$ : C, 70.74; H, 7.92. Found: C, 70.89; H, 7.90.

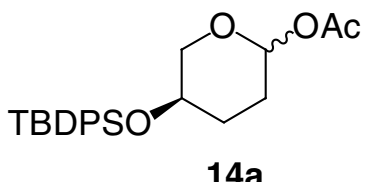

4-(tert-Butyldiphenylsilyloxy)tetrahydropyran-1-acetate (14a): A solution of 4-(tertbutyldiphenylsilyloxy)tetrahydropyran-1-ol $(0.411 \mathrm{~g}, 1.15 \mathrm{mmol})$, acetic anhydride $(0.544 \mathrm{~mL}$, $5.76 \mathrm{mmol})$, pyridine $(0.373 \mathrm{~mL}, 4.61 \mathrm{mmol})$ and 4-dimethylaminopyridine $(0.170 \mathrm{~g}, 1.38$ mmol) in $\mathrm{CH}_{2} \mathrm{Cl}_{2}(20 \mathrm{~mL})$ was stirred for $24 \mathrm{~h}$. Saturated aqueous $\mathrm{NH}_{4} \mathrm{Cl}(10 \mathrm{~mL})$ was added, and $\mathrm{CH}_{2} \mathrm{Cl}_{2}$ was removed in vacuo. The residue was dissolved in MTBE $(75 \mathrm{~mL})$, and the layers were separated. The organic layer was washed with saturated aqueous $\mathrm{Na}_{2} \mathrm{HPO}_{4}$ (4 $\times 20 \mathrm{~mL}), \mathrm{NaH}_{2} \mathrm{PO}_{4}(4 \times 20 \mathrm{~mL})$, and $\mathrm{CuSO}_{4}(5 \times 20 \mathrm{~mL})$, filtered through $\mathrm{Na}_{2} \mathrm{SO}_{4}$, and concentrated in vacuo. The resulting oil was purified by flash chromatography (1:9 to $1: 2$ EtOAc /hexanes) to afford the product (as a 1.1:1 mixture of anomers) as a colorless oil (0.402 
$\mathrm{g}, 88 \%):{ }^{3} \mathrm{H}$ NMR $\left(500 \mathrm{MHz}, \mathrm{CDCl}_{3}\right) \delta 7.68-7.64(\mathrm{~m}, 8.4 \mathrm{H}), 7.45-7.35(\mathrm{~m}, 12.6 \mathrm{H}), 5.98(\mathrm{t}, J$ $=3.6,1.1 \mathrm{H}), 5.88(\mathrm{t}, J=2.5,1 \mathrm{H}), 3.79-3.71(\mathrm{~m}, 3.2 \mathrm{H}), 3.61-3.53(\mathrm{~m}, 2 \mathrm{H}), 3.47(\mathrm{ddd}, J=11.8$, 4.4, 1.4, 1.1H), $2.23(\mathrm{~m}, 1.1 \mathrm{H}), 2.12(\mathrm{~s}, 3 \mathrm{H}), 2.05(\mathrm{~s}, 3.3 \mathrm{H}), 1.93-1.78(\mathrm{~m}, 4.2 \mathrm{H}), 1.68-1.51(\mathrm{~m}$, 3.1H), 1.07 (s, 9H), 1.06 (s, 9.9H); ${ }^{13} \mathrm{C}$ NMR (125 MHz, $\mathrm{CDCl}_{3}$ ) $\delta 169.80,169.78,135.7$, $135.63,135.56,133.9,133.8,133.7,129.82,129.76,129.7,127.68,127.65,127.63,127.57$, 92.4, 90.6, 67.4, 66.5, 66.4, 65.7, 27.7, 27.5, 26.9, 26.6, 24.9, 21.2, 21.1, 19.2, 19.1; IR (thin film) $2959,1749,1428,1008,705 \mathrm{~cm}^{-1}$; HRMS (Cl/isobutane) $\mathrm{m} / z$ calcd for $\mathrm{C}_{21} \mathrm{H}_{27} \mathrm{O}_{2} \mathrm{Si}(\mathrm{M}-$ $\left.\mathrm{C}_{2} \mathrm{H}_{3} \mathrm{O}_{2}\right)^{+} 339.1780$, found 339.1780. Anal. Calcd for $\mathrm{C}_{23} \mathrm{H}_{30} \mathrm{O}_{4} \mathrm{Si}$ : C, 69.31; H, 7.59. Found: C, 69.35; H, 7.60.

\section{IIB. Synthesis of 4-Methanesulfoxytetrahydropyran-1-acetate (14b)}
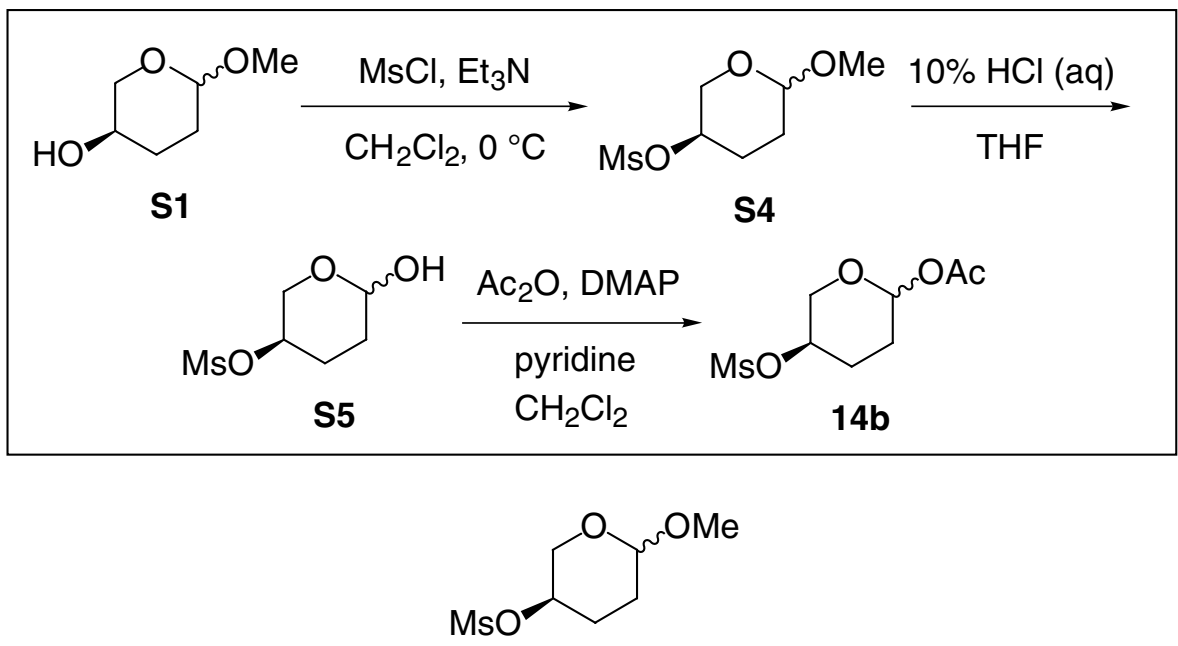

S4

4-Methanesulfoxy-1-methoxytetrahydropyran (S4): Alcohol 4-hydroxy-1methoxytetrahydropyran was dissolved in $\mathrm{CH}_{2} \mathrm{Cl}_{2}(183 \mathrm{~mL})$ and cooled to $0{ }^{\circ} \mathrm{C}$. Triethylamine $(10.1 \mathrm{~mL}, 72.6 \mathrm{mmol})$ was added, followed by slow addition of methanesulfonyl chloride (6.56 $\mathrm{mL}, 84.7 \mathrm{mmol}$ ). The mixture was stirred at $0{ }^{\circ} \mathrm{C}$ for $3 \mathrm{~h}$ and poured into a solution of $1 \mathrm{~N} \mathrm{HCl}$ $(300 \mathrm{~mL})$. The layers were separated, and the aqueous layer was extracted with $\mathrm{Et}_{2} \mathrm{O}(3 \times 100$ $\mathrm{mL})$. The combined organic layers were washed with saturated aqueous $\mathrm{NaHCO}_{3}(200 \mathrm{~mL})$, dried over $\mathrm{MgSO}_{4}$, filtered and concentrated in vacuo. The resulting oil was purified by flash 
chromatography (1:4 to $3: 2 \mathrm{Et}_{2} \mathrm{O} /$ pentane) to afford the product (as a 1.4:1 mixture of anomers) as a colorless oil $(11.85 \mathrm{~g}, 93 \%):{ }^{3}{ }^{1} \mathrm{H} \mathrm{NMR}\left(500 \mathrm{MHz}, \mathrm{CDCl}_{3}\right) \delta 4.77$ (br s, 1.4H), 4.72-4.68 $(\mathrm{m}, 2.4 \mathrm{H}), 4.55(\mathrm{t}, J=3.2,1 \mathrm{H}), 3.94(\mathrm{dd}, J=12.9,0.6,1.4 \mathrm{H}), 3.82-3.73(\mathrm{~m}, 3.4 \mathrm{H}), 3.396(\mathrm{~s}$, $3 \mathrm{H}), 3.392(\mathrm{~s}, 4.2 \mathrm{H}), 3.08(\mathrm{~s}, 4.2 \mathrm{H}), 3.04(\mathrm{~s}, 3 \mathrm{H}), 2.19-1.85(\mathrm{~m}, 7.2 \mathrm{H}), 1.77(\mathrm{~m}, 1 \mathrm{H}), 1.62(\mathrm{~m}$, $1.4 \mathrm{H}) ;{ }^{13} \mathrm{C} \mathrm{NMR}\left(125 \mathrm{MHz}, \mathrm{CDCl}_{3}\right) \delta 97.8,97.7,77.2,75.0,74.1,62.3,61.6,55.0,38.8,38.6$, 28.0, 25.576, 25.582, 23.4; IR (thin film) 2940, 1443, 1352, $920 \mathrm{~cm}^{-1}$; HRMS (CI/isobutane) $\mathrm{m}$ / $z$ calcd for $\mathrm{C}_{7} \mathrm{H}_{13} \mathrm{O}_{5} \mathrm{~S}(\mathrm{M}-\mathrm{H})^{+}$209.0484, found 209.0488. Anal. Calcd for $\mathrm{C}_{7} \mathrm{H}_{14} \mathrm{O}_{5} \mathrm{~S}$ : C, 39.99; H, 6.71. Found: C, 40.12; H, 6.61.

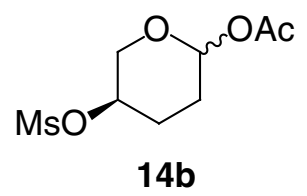

4-Methanesulfoxytetrahydropyran-1-acetate (14b): An aqueous (10\%) solution of $\mathrm{HCl}$ $(100 \mathrm{~mL})$ was added slowly to a solution of 4-methanesulfoxy-1-methoxytetrahydropyran $(2.90$ $\mathrm{g}, 13.8 \mathrm{mmol})$ in THF (200 mL). After $12 \mathrm{~h}$, the mixture was neutralized with saturated aqueous $\mathrm{NaHCO}_{3}$. The solvent was removed in vacuo, and the remaining aqueous layer was extracted with $\mathrm{Et}_{2} \mathrm{O}(4 \times 150 \mathrm{~mL})$, dried over $\mathrm{MgSO}_{4}$, filtered, and concentrated in vacuo to afford the product as a mixture of closed hemiacetal and open aldehyde isomers. A small amount of residual starting material $(0.987 \mathrm{~g}, 4.69 \mathrm{mmol})$ was removed by flash chromatography (1:9 to 9:1 $\mathrm{Et}_{2} \mathrm{O} /$ pentane). A solution of the hemiacetal/aldehyde mixture $(0.558 \mathrm{~g}, 2.84 \mathrm{mmol})$, acetic anhydride $(1.34 \mathrm{~mL}, 14.2 \mathrm{mmol})$, pyridine $(0.920 \mathrm{~mL}, 11.4 \mathrm{mmol})$ and 4-dimethylaminopyridine $(0.419 \mathrm{~g}, 3.41 \mathrm{mmol})$ in $\mathrm{CH}_{2} \mathrm{Cl}_{2}(14 \mathrm{~mL})$ was stirred for $24 \mathrm{~h}$. Saturated aqueous $\mathrm{NH}_{4} \mathrm{Cl}(10 \mathrm{~mL})$ was added, and $\mathrm{CH}_{2} \mathrm{Cl}_{2}$ was removed in vacuo. The residue was dissolved in MTBE (100 mL), and the layers were separated. The organic layer was washed with saturated aqueous $\mathrm{Na}_{2} \mathrm{HPO}_{4}(4 \times 25 \mathrm{~mL}), \mathrm{NaH}_{2} \mathrm{PO}_{4}(4 \times 25 \mathrm{~mL})$, and $\mathrm{CuSO}_{4}(5 \times 25 \mathrm{~mL})$, filtered through $\mathrm{Na}_{2} \mathrm{SO}_{4}$, and concentrated in vacuo. The resulting oil was purified by flash chromatography (9:1 to $1: 4$ pentane/ $\mathrm{Et}_{2} \mathrm{O}$ ) to afford the product (as a 1.1:1 mixture of anomers) 
as a colorless oil. The product was prone to hydrolysis and elimination, and decomposition was observed upon concentration and warming above $23{ }^{\circ} \mathrm{C}(0.643 \mathrm{~g}, 30 \%$ over two steps, based on recovered starting material): ${ }^{1} \mathrm{H} \mathrm{NMR}\left(500 \mathrm{MHz}, \mathrm{CDCl}_{3}\right) \delta 6.11(\mathrm{t}, J=2.3,1 \mathrm{H}), 5.94(\mathrm{t}, J=$ $3.5,1.1 \mathrm{H}), 4.84(\mathrm{~m}, 1 \mathrm{H}), 4.74(\mathrm{ddd}, J=13.4,7.5,4.9,1.1 \mathrm{H}), 4.03(\mathrm{dd}, J=13.2,1.7,1 \mathrm{H})$, 3.92-3.84 (m, 3.2H), 3.10 (s, 3H), 3.09 (s, 3.3H), 2.21-2.15 (m, 2H), 2.14-2.10 (m, 8.5H), 2.06 (m, 1H), $1.97(\mathrm{~m}, 1.1 \mathrm{H}), 1.88(\mathrm{~m}, 1.1 \mathrm{H}), 1.70(\mathrm{~m}, 1 \mathrm{H}) ;{ }^{13} \mathrm{C} \mathrm{NMR}\left(125 \mathrm{MHz}, \mathrm{CDCl}_{3}\right) \delta 169.4$, $169.3,90.8,90.5,73.8,73.2,64.2,63.5,38.9,38.7,26.8,25.6,23.4,23.2,21.01,20.97$; IR (thin film) $2942,1747,1348,1172,920 \mathrm{~cm}^{-1}$; HRMS (CI/isobutene) $\mathrm{m} / z$ calcd for $\mathrm{C}_{6} \mathrm{H}_{11} \mathrm{O}_{5} \mathrm{~S}(\mathrm{M}-$ $\left.\mathrm{C}_{2} \mathrm{H}_{3} \mathrm{O}\right)^{+}$195.0327, found 195.0327 .

\section{IIC. Synthesis of 4-Benzoyloxytetrahyropyran-1-acetate (14c)}

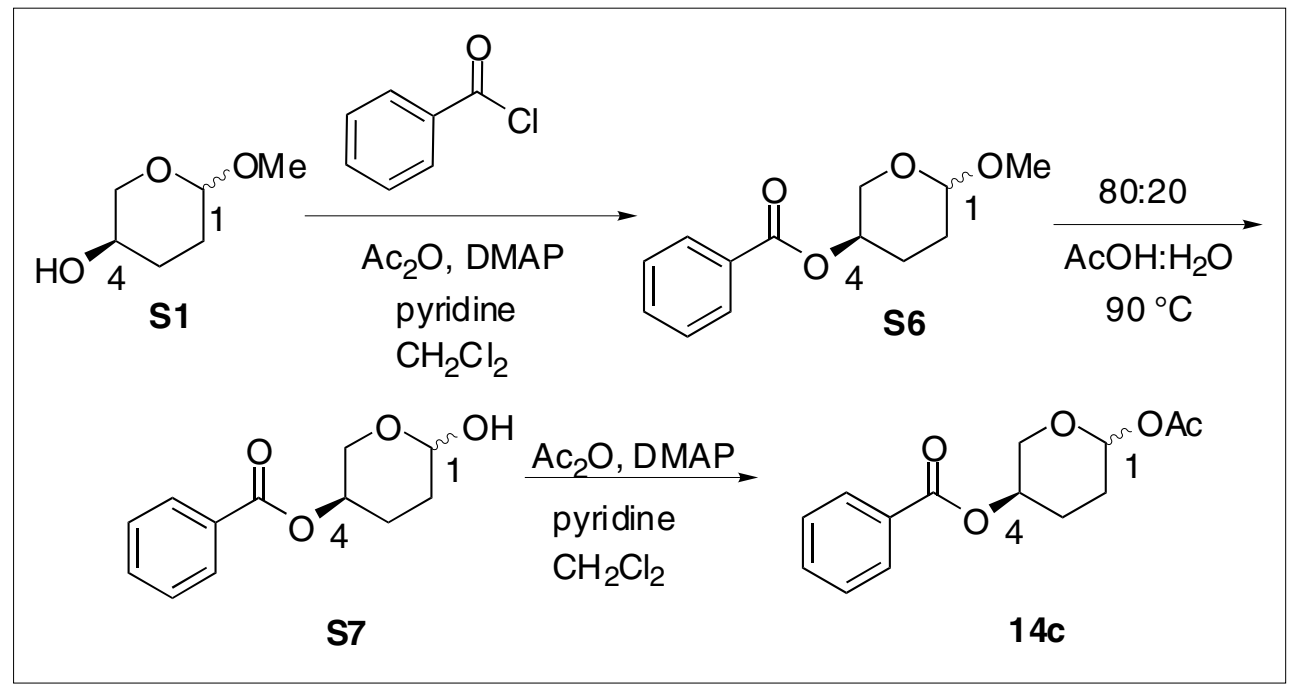<smiles>CO[C@H]1CC[C@@H](OC(=O)c2ccccc2)CO1</smiles>

4-Benzoyloxy-1-methoxytetrahydropyran (S6): $\quad$ To a solution of 4-hydroxy-1methoxytetrahydropyran $(0.798 \mathrm{~g}, 5.96 \mathrm{mmol})$ in $\mathrm{CH}_{2} \mathrm{Cl}_{2}(32 \mathrm{~mL})$ was added benzoyl chloride 
(0.692 mL, $5.96 \mathrm{mmol})$, pyridine $(1.90 \mathrm{~mL}, 23.8 \mathrm{mmol})$, and 4-dimethylaminopyridine (1.60 g, 7.15 mmol). The reaction mixture was allowed to stir at $23{ }^{\circ} \mathrm{C}$ for $15 \mathrm{~h}$. Ethylenediamine $(12 \mathrm{~mL})$ was added, and the mixture was stirred for $2 \mathrm{~h}$. Saturated aqueous $\mathrm{NH}_{4} \mathrm{Cl}(16 \mathrm{~mL})$ was added, and the $\mathrm{CH}_{2} \mathrm{Cl}_{2}$ was removed in vacuo. The residue was dissolved in MTBE (30 mL), and the layers were separated. The organic layer was washed with saturated aqueous $\mathrm{Na}_{2} \mathrm{HPO}_{4}(3 \times 50 \mathrm{~mL}), \mathrm{NaH}_{2} \mathrm{PO}_{4}$ $(3 \times 50 \mathrm{~mL})$, and $\mathrm{CuSO}_{4}(4 \times 50 \mathrm{~mL})$, filtered through $\mathrm{Na}_{2} \mathrm{SO}_{4}$, and concentrated in vacuo. The resulting oil was purified by flash column chromatography (hexanes to 1:9 EtOAc/hexanes) to afford the product (as a 1:2 mixture of isomers) as a colorless oil $(1.25 \mathrm{~g}, 89 \%):{ }^{3} \mathrm{H}$ NMR (500 $\left.\mathrm{MHz}, \mathrm{CDCl}_{3}\right) \delta 8.09(\mathrm{~m}, 2 \mathrm{H}), 8.04(\mathrm{~m}, 2 \mathrm{H}), 7.57(\mathrm{~m}, 3 \mathrm{H}), 7.46(\mathrm{~m}, 3 \mathrm{H}), 5.08(\mathrm{~m}, 2 \mathrm{H}), 4.72(\mathrm{~m}$, 2H), $4.04(\mathrm{dd}, J=12.6,2.0,2 \mathrm{H}), 3.85(\mathrm{~m}, 1 \mathrm{H}), 3.74(\mathrm{~m}, 1 \mathrm{H}), 3.44(\mathrm{~s}, 3 \mathrm{H}), 3.42(\mathrm{~s}, 3 \mathrm{H}), 2.22(\mathrm{~m}$, 2H), $2.02(\mathrm{~m}, 2 \mathrm{H}), 1.86(\mathrm{~m}, 2 \mathrm{H}), 1.66(\mathrm{~m}, 2 \mathrm{H}) ;{ }^{13} \mathrm{C} \mathrm{NMR}\left(125 \mathrm{MHz}, \mathrm{CDCl}_{3}\right) \delta$ 166.0, 165.8, $133.01,132.95,130.5,129.7,128.3,98.6,98.2,68.1,67.9,62.7,61.8,55.2,55.0,28.1,25.4,24.8$, 22.5; IR (thin film) 3624, 2939, 1724, 1602, $1274 \mathrm{~cm}^{-1}$; HRMS (EI/GCMS) $m / z$ calcd for $\mathrm{C}_{12} \mathrm{H}_{13} \mathrm{O}_{3}\left(\mathrm{M}-\mathrm{CH}_{3} \mathrm{O}\right)^{+}$205.0865, found 205.0868. Anal. Calcd for $\mathrm{C}_{13} \mathrm{H}_{16} \mathrm{O}_{4}: \mathrm{C}, 66.09 ; \mathrm{H}, 6.83$. Found: C, 65.14; H, 6.83.

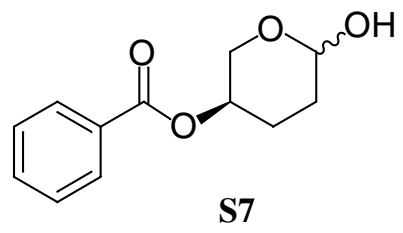

\section{4-Benzoyloxytetrahydropyran-1-ol (S7): $\quad$ A mixture of 4-benzoyl-1-} methoxytetrahydropyran $(1.50 \mathrm{~g}, 6.35 \mathrm{mmol})$ and 80:20 AcOH: $\mathrm{H}_{2} \mathrm{O}(65 \mathrm{~mL})$ was stirred at 90 ${ }^{\circ} \mathrm{C}$ for $24 \mathrm{~h}$. The reaction mixture was cooled to $23{ }^{\circ} \mathrm{C}$ and the solvent was removed in vacuo as an azeotrope with toluene. The resulting oil was purified by flash column chromatography (hexanes to 1:3 EtOAc/hexanes) to afford the product (as a 1:1 mixture of isomers) as a colorless oil $(1.15 \mathrm{~g}, 82 \%):{ }^{3} \mathrm{H}$ NMR $\left(500 \mathrm{MHz}, \mathrm{CDCl}_{3}\right) \delta 8.07(\mathrm{~m}, 4 \mathrm{H}), 7.57(\mathrm{~m}, 2 \mathrm{H}), 7.45$ 
$(\mathrm{m}, 4 \mathrm{H}), 5.28(\mathrm{~m}, 1 \mathrm{H}), 5.06(\mathrm{~m}, 3 \mathrm{H}), 4.26(\mathrm{dd}, J=12.3,2.1,1 \mathrm{H}), 4.09(\mathrm{dd}, J=12.3,6.5,1 \mathrm{H})$, $3.80(\mathrm{dd}, J=12.3,2.1,1 \mathrm{H}), 3.71(\mathrm{~m}, 1 \mathrm{H}), 2.27(\mathrm{~m}, 1 \mathrm{H}), 2.10(\mathrm{~m}, 2 \mathrm{H}), 1.85(\mathrm{~m}, 4 \mathrm{H}), 1.68(\mathrm{~m}$, $1 \mathrm{H}) ;{ }^{13} \mathrm{C} \mathrm{NMR}\left(125 \mathrm{MHz}, \mathrm{CDCl}_{3}\right) \delta 166.0,165.9,133.0,132.9,129.6,129.5,128.2,93.5,92.2$, 67.9, 67.4, 64.7, 62.5, 28.4, 26.5, 25.2, 22.8; IR (thin film) 3424, 2956, 1719, $1602 \mathrm{~cm}^{-1}$; HRMS (EI/GCMS) $m / z$ calcd for $\mathrm{C}_{12} \mathrm{H}_{14} \mathrm{NaO}_{4}(\mathrm{M}+\mathrm{Na})^{+} 245.0790$, found 245.0793. Anal. Calcd for $\mathrm{C}_{12} \mathrm{H}_{14} \mathrm{O}_{4}: \mathrm{C}, 64.85 ; \mathrm{H}, 6.35$. Found: C, 64.64; H, 6.46.

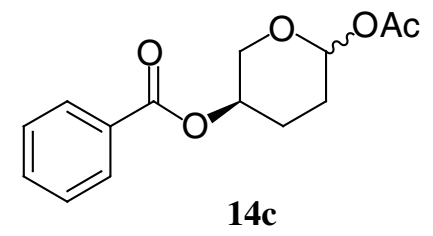

4-Benzoyloxytetrahydropyran-1-acetate (14c): To a stirring solution of 4-benzoyltetrahydropyran-1-ol $(0.972 \mathrm{~g}, 4.37 \mathrm{mmol})$ in $\mathrm{CH}_{2} \mathrm{Cl}_{2}(44 \mathrm{~mL})$ was added pyridine $(1.40 \mathrm{~mL}$, $17.5 \mathrm{mmol})$ followed by 4-dimethylaminopyridine $(1.19 \mathrm{~g}, 5.25 \mathrm{mmol})$. Acetic anhydride (2.06 $\mathrm{mL}, 21.9 \mathrm{mmol}$ ) was added drop wise to the solution by syringe, and the mixture was stirred for 38 h. Saturated aqueous $\mathrm{NH}_{4} \mathrm{Cl}(30 \mathrm{~mL})$ was added, and the $\mathrm{CH}_{2} \mathrm{Cl}_{2}$ was removed in vacuo. The residue was dissolved in MTBE $(150 \mathrm{~mL})$, and the organic layer was washed with saturated aqueous $\mathrm{Na}_{2} \mathrm{HPO}_{4}(4 \times 50 \mathrm{~mL}), \mathrm{NaH}_{2} \mathrm{PO}_{4}(4 \times 50 \mathrm{~mL})$, and $\mathrm{CuSO}_{4}(5 \times 50 \mathrm{~mL})$, filtered through $\mathrm{Na}_{2} \mathrm{SO}_{4}$, and concentrated in vacuo. The resulting oil was purified by flash column chromatography (hexanes to 1:9 EtOAc/hexanes) to afford the product (as a 1:1 mixture of diastereomers) as a colorless oil (0.913 g, 79\%): ${ }^{1}{ }^{1} \mathrm{H}$ NMR $\left(500 \mathrm{MHz}, \mathrm{C}_{6} \mathrm{D}_{6}\right) \delta 8.07(\mathrm{~m}, 4 \mathrm{H})$, $7.57(\mathrm{~m}, 2 \mathrm{H}), 7.45(\mathrm{~m}, 4 \mathrm{H}), 6.16(\mathrm{bs}, 1 \mathrm{H}), 5.98(\mathrm{t}, J=3.6,1 \mathrm{H}), 5.14(\mathrm{~m}, 2 \mathrm{H}), 4.10(\mathrm{dd}, J=12.7$, 1.9, 1H), $3.91(\mathrm{~m}, 3 \mathrm{H}), 2.20(\mathrm{~m}, 2 \mathrm{H}), 2.14(\mathrm{~s}, 3 \mathrm{H}), 2.13(\mathrm{~s}, 3 \mathrm{H}), 1.98(\mathrm{~m}, 5 \mathrm{H}), 1.73(\mathrm{~m}, 1 \mathrm{H}) ;{ }^{13} \mathrm{C}$ NMR $\left(125 \mathrm{MHz}, \mathrm{CDCl}_{3}\right) \delta 170.3,170.1,166.5,166.2,133.73,133.70,130.7,130.5,130.2$, 129.0, 128.9, 91.9, 91.8, 67.80, 67.77, 64.9, 64.2, 27.5, 25.2, 24.6, 22.7, 21.71, 21.69; IR (thin film) 2961, 1748, $1719 \mathrm{~cm}^{-1}$; HRMS (EI/GCMS) $m / z$ calcd for $\mathrm{C}_{12} \mathrm{H}_{13} \mathrm{O}_{3}\left(\mathrm{M}-\mathrm{C}_{2} \mathrm{H}_{3} \mathrm{O}_{2}\right)^{+}$ 
205.0865, found 205.0868. Anal. calcd for $\mathrm{C}_{14} \mathrm{H}_{16} \mathrm{O}_{5}$ : C, 63.63; H, 6.10. Found: C, 63.70; H, 6.21 .

\section{IID. Synthesis of 4-(p-Anisoyloxy)tetrahydropyran-1-acetate (14d)}

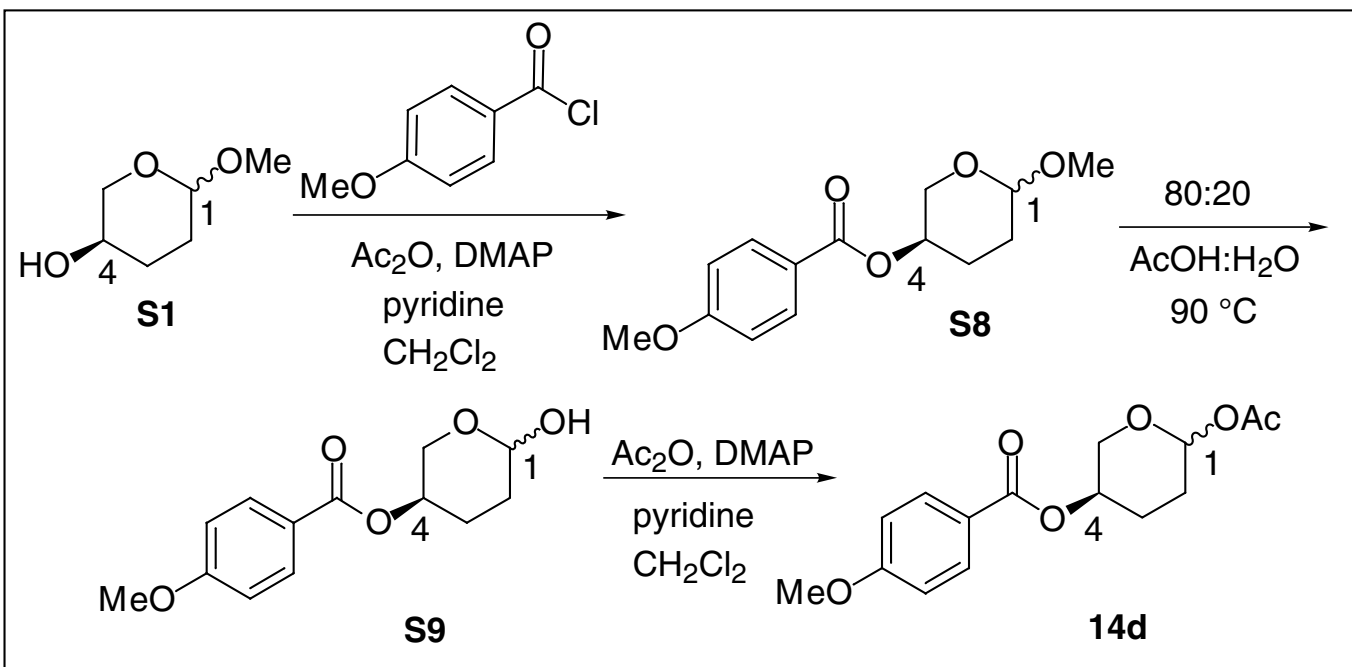<smiles>COc1ccc(C(=O)OC2CCC(OC)OC2)cc1</smiles>

4-(p-Anisoyloxy)-1-methoxytetrahydropyran (S8): To a solution of 4-hydroxy-1methoxytetrahydropyran $(0.050 \mathrm{~g}, 0.38 \mathrm{mmol})$ in $\mathrm{CH}_{2} \mathrm{Cl}_{2}(3 \mathrm{~mL})$ was added $p$-anisoyl chloride (0.065 g, $0.38 \mathrm{mmol})$, pyridine $(0.121 \mathrm{~mL}, 1.51 \mathrm{mmol})$, and 4-dimethylaminopyridine (0.102 $\mathrm{g}$, $0.454 \mathrm{mmol})$. The reaction mixture was allowed to stir at $23{ }^{\circ} \mathrm{C}$ for $15 \mathrm{~h}$. Ethylenediamine $(0.750 \mathrm{~mL})$ was added, and the mixture was stirred for $1 \mathrm{~h}$. Saturated aqueous $\mathrm{NH}_{4} \mathrm{Cl}(10 \mathrm{~mL})$ was added, and the $\mathrm{CH}_{2} \mathrm{Cl}_{2}$ was removed in vacuo. The residue was dissolved in MTBE (15 $\mathrm{mL}$ ), and the layers were separated. The organic layer was washed with saturated aqueous $\mathrm{Na}_{2} \mathrm{HPO}_{4}(3 \times 25 \mathrm{~mL}), \mathrm{NaH}_{2} \mathrm{PO}_{4}(3 \times 25 \mathrm{~mL})$, and $\mathrm{CuSO}_{4}(4 \times 25 \mathrm{~mL})$, filtered through 
$\mathrm{Na}_{2} \mathrm{SO}_{4}$, and concentrated in vacuo. The resulting oil was purified by flash column chromatography (hexanes to 1:9 EtOAc/hexanes) to afford the product (as a 1:1 mixture of isomers) as a colorless oil $(0.070 \mathrm{~g}, 69 \%):{ }^{1} \mathrm{H}$ NMR $\left(500 \mathrm{MHz}, \mathrm{CDCl}_{3}\right) \delta 7.94(\mathrm{~m}, 4 \mathrm{H}), 6.85$ $(\mathrm{m}, 4 \mathrm{H}), 4.97(\mathrm{~m}, 2 \mathrm{H}), 4.64(\mathrm{~m}, 2 \mathrm{H}), 3.92(\mathrm{dd}, J=12.5,4.6,1 \mathrm{H}), 3.79(\mathrm{~s}, 3 \mathrm{H}), 3.78(\mathrm{~s}, 3 \mathrm{H}), 3.65$ $(\mathrm{m}, 2 \mathrm{H}), 3.37(\mathrm{~m}, 1 \mathrm{H}), 3.36(\mathrm{~s}, 3 \mathrm{H}), 3.35(\mathrm{~s}, 3 \mathrm{H}), 2.07(\mathrm{~m}, 2 \mathrm{H}), 1.93-1.76(\mathrm{~m}, 3 \mathrm{H}), 1.58(\mathrm{~m}$, $3 \mathrm{H}) ;{ }^{13} \mathrm{C} \mathrm{NMR}\left(125 \mathrm{MHz}, \mathrm{CDCl}_{3}\right) \delta$ 166.2, 163.9, 132.1, 123.0, 114.0, 99.1, 98.7, 68.1, 68.0, 63.2, 62.3, 55.8, 55.5, 55.4, 29.0, 25.9, 25.3, 23.0; IR (thin film) 2957, 1711, 1607, 1511, 1258 $\mathrm{cm}^{-1}$; HRMS (EI/GCMS) $m / z$ calcd for $\mathrm{C}_{14} \mathrm{H}_{18} \mathrm{O}_{5}(\mathrm{M})^{+}$266.1154, found 266.1159. Anal. Calcd for $\mathrm{C}_{14} \mathrm{H}_{18} \mathrm{O}_{5}: \mathrm{C}, 63.15 ; \mathrm{H}, 6.81$. Found: $\mathrm{C}, 63.27 ; \mathrm{H}, 6.83$.

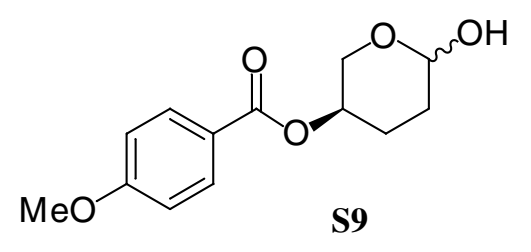

4-(p-Anisoyl)tetrahydropyran-1-ol (S9): $\quad$ A mixture of 4-(p-anisoyl)-1methoxytetrahydropyran $(1.21 \mathrm{~g}, 4.54 \mathrm{mmol})$ and 80:20 AcOH: $\mathrm{H}_{2} \mathrm{O}(40 \mathrm{~mL})$ was stirred at 90 ${ }^{\circ} \mathrm{C}$ for $48 \mathrm{~h}$. The reaction mixture was cooled to $23{ }^{\circ} \mathrm{C}$ and the solvent was removed in vacuo as an azeotrope with toluene. The resulting oil was purified by flash column chromatography (hexanes to 3:7 EtOAc/hexanes) to afford the product (as a 1:1 mixture of isomers) as a colorless oil $(0.872 \mathrm{~g}, 76 \%):{ }^{1} \mathrm{H}$ NMR (500 MHz, $\left.\mathrm{CDCl}_{3}\right) \delta 8.03(\mathrm{~m}, 4 \mathrm{H}), 6.92(\mathrm{~m}, 4 \mathrm{H}), 5.21$ (m, 1H), $5.04(\mathrm{~m}, 3 \mathrm{H}), 4.23(\mathrm{dd}, J=12.6,2.1,1 \mathrm{H}), 4.09$ (ddd, $J=11.8,5.7,1.3,1 \mathrm{H}), 3.86$ (s, $3 \mathrm{H}), 3.85(\mathrm{~s}, 3 \mathrm{H}), 3.78(\mathrm{~m}, 1 \mathrm{H}), 3.70(\mathrm{ddd}, J=12.2,4.0,1.6,1 \mathrm{H}), 2.23(\mathrm{~m}, 1 \mathrm{H}), 2.11(\mathrm{~m}, 2 \mathrm{H})$, $1.85(\mathrm{~m}, 4 \mathrm{H}), 1.65(\mathrm{~m}, 1 \mathrm{H}) ;{ }^{13} \mathrm{C} \mathrm{NMR}\left(125 \mathrm{MHz}, \mathrm{CDCl}_{3}\right) \delta$ 167.0, 164.0, 132.23, 132.19, $123.2,123.1,114.1,94.0,93.0,68.0,67.7,65.3,63.4,55.9,29.1,27.2,25.7,23.5$; IR (thin film) 3417, 2956, 1709, 1607, 1169, $1026 \mathrm{~cm}^{-1}$; HRMS (EI/GCMS) $m / z$ calcd for $\mathrm{C}_{13} \mathrm{H}_{16} \mathrm{O}_{5}(\mathrm{M})^{+}$ 
252.0998, found 252.0990. Anal. Calcd for $\mathrm{C}_{13} \mathrm{H}_{16} \mathrm{O}_{5}$ : C, 61.90; H, 6.39. Found: C, 62.24; $\mathrm{H}$, 6.73.

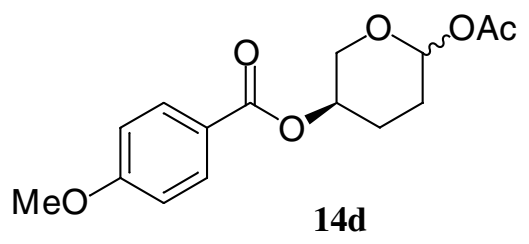

4-(p-Anisoyl)tetrahydropyran-1-acetate $\quad(\mathbf{1 4 d}): \quad$ To $\quad$ a $\quad$ solution $\quad$ of 4 - $(p$ anisoyl)tetrahydropyran-1-ol $(0.391 \mathrm{~g}, 1.55 \mathrm{mmol})$ in $\mathrm{CH}_{2} \mathrm{Cl}_{2}(32 \mathrm{~mL})$ was added pyridine (0.497 mL, $6.20 \mathrm{mmol})$ followed by 4-dimethylaminopyridine $(0.417 \mathrm{~g}, 1.86 \mathrm{mmol})$. Acetic anhydride $(0.731 \mathrm{~mL}, 7.75 \mathrm{mmol})$ was added drop wise to the solution by syringe, and the mixture was stirred for $38 \mathrm{~h}$. Saturated aqueous $\mathrm{NH}_{4} \mathrm{Cl}(25 \mathrm{~mL})$ was added, and the $\mathrm{CH}_{2} \mathrm{Cl}_{2}$ was removed in vacuo. The residue was dissolved in MTBE $(20 \mathrm{~mL})$, and the organic layer was washed with saturated aqueous $\mathrm{Na}_{2} \mathrm{HPO}_{4}(4 \times 15 \mathrm{~mL}), \mathrm{NaH}_{2} \mathrm{PO}_{4}(4 \times 15 \mathrm{~mL})$, and $\mathrm{CuSO}_{4}(5 \times$ $15 \mathrm{~mL}$ ), filtered through $\mathrm{Na}_{2} \mathrm{SO}_{4}$, and concentrated in vacuo. The resulting oil was purified by flash column chromatography (hexanes to 1:9 EtOAc/hexanes) to afford the product (as a 1:1 mixture of diastereomers) as a colorless oil $(0.366 \mathrm{~g}, 80 \%):{ }^{31} \mathrm{H}$ NMR $\left(500 \mathrm{MHz}, \mathrm{CDCl}_{3}\right) \delta$ $8.03(\mathrm{~m}, 4 \mathrm{H}), 6.93(\mathrm{~m}, 4 \mathrm{H}), 6.16(\mathrm{~m}, 1 \mathrm{H}), 5.99(\mathrm{t}, J=3.6,1 \mathrm{H}), 5.11(\mathrm{~m}, 1 \mathrm{H}), 5.06(\mathrm{~m}, 1 \mathrm{H}), 4.08$ $(\mathrm{dd}, J=12.7,1.9,1 \mathrm{H}), 3.91(\mathrm{~m}, 3 \mathrm{H}), 3.87(\mathrm{~s}, 3 \mathrm{H}), 3.86(\mathrm{~s}, 3 \mathrm{H}), 2.24(\mathrm{~m}, 2 \mathrm{H}), 2.13(\mathrm{~s}, 3 \mathrm{H}), 2.12$ (s, 3H), 2.05 (m, 3H), $1.96(\mathrm{~m}, 2 \mathrm{H}), 1.73$ (m, 1H); IR (thin film) 2961, 2939, 1748, 1711, 1607, $1512 \mathrm{~cm}^{-1}$; HRMS (EI/GCMS) $m / z$ calcd for $\mathrm{C}_{13} \mathrm{H}_{15} \mathrm{O}_{4}\left(\mathrm{M}-\mathrm{C}_{2} \mathrm{H}_{3} \mathrm{O}_{2}\right)^{+}$235.0970, found 235.0969. Anal. Calcd for $\mathrm{C}_{15} \mathrm{H}_{18} \mathrm{O}_{6}$ : C, 61.22; H, 6.16. Found: C, 61.48; H, 6.21. 


\section{IIE. Synthesis of 4-( $p$-Nitrobenzoyloxy)tetrahydropyran-1-acetate (14e)}
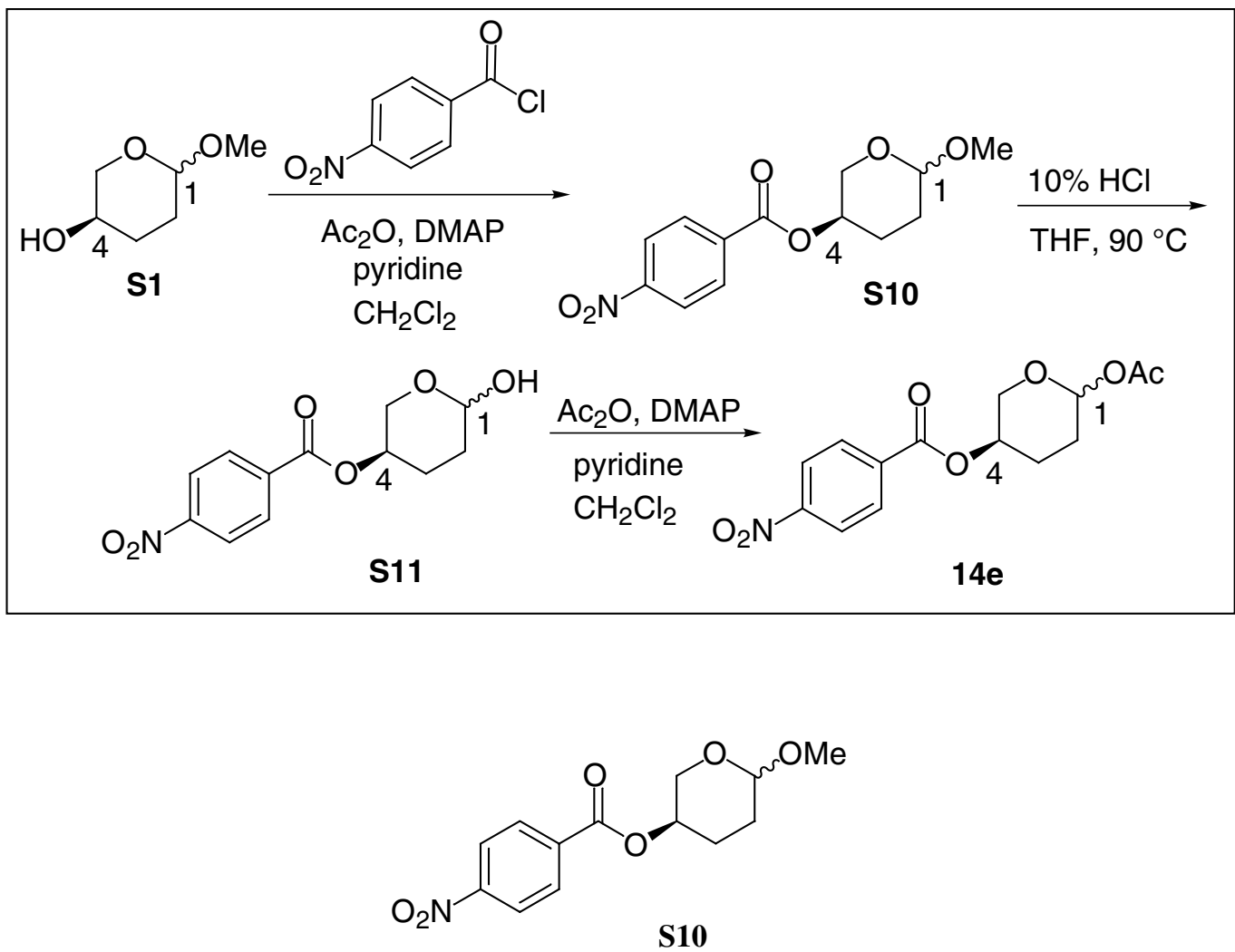

4-(p-Nitrobenzoyloxy)-1-methoxytetrahydropyran (S10): To a solution of 4-hydroxy-1methoxytetrahydropyran $(0.506 \mathrm{~g}, 3.83 \mathrm{mmol})$ in $\mathrm{CH}_{2} \mathrm{Cl}_{2}(20 \mathrm{~mL})$ was added 4-nitrobenzoyl chloride (0.711 g, $3.83 \mathrm{mmol})$, pyridine $(1.23 \mathrm{~mL}, 15.3 \mathrm{mmol})$, and 4-dimethylaminopyridine $(0.561 \mathrm{~g}, 4.59 \mathrm{mmol})$. The reaction mixture was allowed to stir at $23{ }^{\circ} \mathrm{C}$ for $15 \mathrm{~h}$. Saturated aqueous $\mathrm{NH}_{4} \mathrm{Cl}(25 \mathrm{~mL})$ was added, and the $\mathrm{CH}_{2} \mathrm{Cl}_{2}$ was removed in vacuo. The residue was dissolved in MTBE (40 mL), and the layers were separated. The organic layer was washed with saturated aqueous $\mathrm{Na}_{2} \mathrm{HPO}_{4}(3 \times 50 \mathrm{~mL}), \mathrm{NaH}_{2} \mathrm{PO}_{4}(3 \times 50 \mathrm{~mL})$, and $\mathrm{CuSO}_{4}(4 \times 50 \mathrm{~mL})$, filtered through $\mathrm{Na}_{2} \mathrm{SO}_{4}$, and concentrated in vacuo. The resulting oil was purified by flash column chromatography (hexanes to 1:9 EtOAc/hexanes) to afford the product as a colorless oil $(0.909 \mathrm{~g}, 84 \%):{ }^{1} \mathrm{H}$ NMR (500 MHz, $\left.\mathrm{CDCl}_{3}\right) \delta 8.29(\mathrm{~m}, 4 \mathrm{H}), 5.17(\mathrm{~m}, 1 \mathrm{H}), 4.80(\mathrm{~m}, 1 \mathrm{H}), 4.09$ (dd, $J=12.7,1.9,1 \mathrm{H}), 3.84(\mathrm{dd}, J=12.7,2.3,1 \mathrm{H}), 3.40(\mathrm{~s}, 3 \mathrm{H}), 2.28(\mathrm{~m}, 1 \mathrm{H}), 2.17(\mathrm{~m}, 1 \mathrm{H})$, 
$1.97(\mathrm{~m}, 1 \mathrm{H}), 1.76(\mathrm{~m}, 1 \mathrm{H}) ;{ }^{13} \mathrm{C}$ NMR $\left(125 \mathrm{MHz}, \mathrm{CDCl}_{3}\right) \delta 164.6,151.1,136.3,131.2,124.0$ 98.4, 69.8, 61.8, 55.5, 25.7, 22.8; IR (thin film) 2957, 1726, 1712, 1517, $1280 \mathrm{~cm}^{-1}$; HRMS (EI/GCMS) $m / z$ calcd for $\mathrm{C}_{13} \mathrm{H}_{16} \mathrm{NO}_{6}(\mathrm{M}+\mathrm{H})^{+}$282.0977, found 282.0969. Anal. Calcd for $\mathrm{C}_{13} \mathrm{H}_{15} \mathrm{NO}_{6}: \mathrm{C}, 55.51 ; \mathrm{H}, 5.38 ; \mathrm{N}, 4.98$. Found: C, 55.74; H, 5.47; N, 4.87.

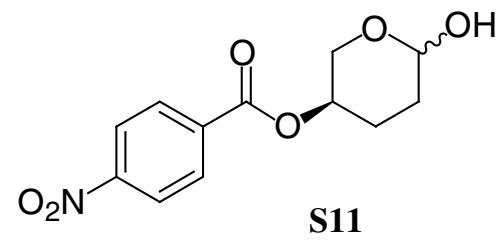

4-( $p$-Nitrobenzoyloxy)tetrahydropyran-1-ol (S11): A 10\% aqueous solution of $\mathrm{HCl}$ (5 $\mathrm{mL}$ ) was added slowly to a solution of 4-(p-nitrobenzoyloxy)-1-methoxytetrahydropyran (0.105 $\mathrm{g}, 0.395 \mathrm{mmol})$ in THF $(10 \mathrm{~mL})$. The reaction mixture was stirred at $90{ }^{\circ} \mathrm{C}$ for $18 \mathrm{~h}$ and allowed to cool to $23{ }^{\circ} \mathrm{C}$. The mixture was neutralized with saturated aqueous $\mathrm{NaHCO}_{3}(15$ $\mathrm{mL})$, extracted with $\mathrm{Et}_{2} \mathrm{O}(5 \times 25 \mathrm{~mL})$, dried over $\mathrm{MgSO}_{4}$, filtered, and concentrated in vacuo. The colorless oil was purified by flash chromatography (hexanes to 3:7 EtOAc/hexanes) to afford the product (as a 1:1 mixture of isomers) as a colorless oil $(0.093 \mathrm{~g}, 93 \%):{ }^{1} \mathrm{H}$ NMR $\left(500 \mathrm{MHz}, \mathrm{CDCl}_{3}\right) \delta 8.30(\mathrm{~m}, 8 \mathrm{H}), 5.27(\mathrm{~m}, 1 \mathrm{H}), 5.13-5.04(\mathrm{~m}, 3 \mathrm{H}), 4.27(\mathrm{dd}, J=12.4,2.3$, $1 \mathrm{H}), 4.16(\mathrm{dd}, J=12.1,5.4,1 \mathrm{H}), 3.85(\mathrm{dd}, J=12.0,3.0,1 \mathrm{H}), 3.75(\mathrm{dt}, J=12.4,1.6,1 \mathrm{H}), 2.33$ (m, 1H), 2.21-2.01 (m, 3H), $1.90(\mathrm{~m}, 3 \mathrm{H}), 1.74(\mathrm{~m}, 3 \mathrm{H}) ;{ }^{13} \mathrm{C} \mathrm{NMR}\left(125 \mathrm{MHz}, \mathrm{CDCl}_{3}\right) \delta 164.4$, 164.3, 150.9, 150.8, 135.9, 135.8, 131.1, 131.0, 123.78, 123.77, 93.8, 92.3, 69.5, 68.9, 64.9, 62.4, 28.7, 26.5, 25.5, 22.8; IR (thin film) 3405, 2940, 1737, 1705, $1606 \mathrm{~cm}^{-1}$. 


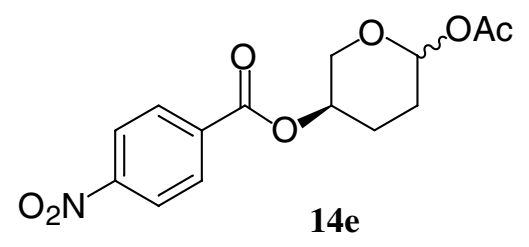

4-(p-Nitrobenzoyloxy)tetrahydropryan-1-acetate $\quad(14 \mathrm{e}): \quad$ To $\quad$ a solution $\quad$ of $\quad 4-(p-$ nitrobenzoyloxy)tetrahydropyran-1-ol $(0.074 \mathrm{~g}, 0.277 \mathrm{mmol})$ in $\mathrm{CH}_{2} \mathrm{Cl}_{2}(5 \mathrm{~mL})$ was added pyridine $(0.889 \mathrm{~mL}, 1.11 \mathrm{mmol})$ followed by 4-dimethylaminopyridine $(0.041 \mathrm{~g}, 0.34 \mathrm{mmol})$. Acetic anhydride $(0.130 \mathrm{~mL}, 1.38 \mathrm{mmol})$ was added drop wise to the solution by syringe, and the mixture was stirred for $44 \mathrm{~h}$. Saturated aqueous $\mathrm{NH}_{4} \mathrm{Cl}(5 \mathrm{~mL})$ was added, and the $\mathrm{CH}_{2} \mathrm{Cl}_{2}$ was removed in vacuo. The residue was dissolved in MTBE $(20 \mathrm{~mL})$, and the organic layer was washed with saturated aqueous $\mathrm{Na}_{2} \mathrm{HPO}_{4}(4 \times 15 \mathrm{~mL}), \mathrm{NaH}_{2} \mathrm{PO}_{4}(4 \times 15 \mathrm{~mL})$, and $\mathrm{CuSO}_{4}(5 \times$ $15 \mathrm{~mL}$ ), filtered through $\mathrm{Na}_{2} \mathrm{SO}_{4}$, and concentrated in vacuo. The resulting oil was purified by flash column chromatography (hexanes to 1:9 EtOAc/hexanes) to afford the product (as a 1:1 mixture of diastereomers) as a colorless oil $(0.054 \mathrm{~g}, 63 \%):{ }^{1} \mathrm{H}$ NMR $\left(500 \mathrm{MHz}, \mathrm{CDCl}_{3}\right) \delta$ 8.31-8.21 (m, 8H), $6.18(\mathrm{~m}, 1 \mathrm{H}), 5.99(\mathrm{~m}, 1 \mathrm{H}), 5.18(\mathrm{~m}, 2 \mathrm{H}), 4.69-4.12(\mathrm{~m}, 2 \mathrm{H}), 4.14(\mathrm{dd}, J=$ $12.9,1.7,1 \mathrm{H}), 3.94(\mathrm{~m}, 1 \mathrm{H}), 2.25-1.75(\mathrm{~m}, 8 \mathrm{H}$ and s, $6 \mathrm{H}) ;{ }^{13} \mathrm{C} \mathrm{NMR}\left(125 \mathrm{MHz}, \mathrm{CDCl}_{3}\right) \delta$ 170.0, 169.9, 164.5, 164.3, 151.1, 136.0, 135.8, 131.3, 124.01, 123.98, 123.95, 91.7, 91.6, 69.0, 68.8, 64.8, 63.8, 27.3, 25.1, 24.5, 22.5, 21.52, 21.50; IR (thin film) 2962, 1722, 1608, 1527, 1348, $1272 \mathrm{~cm}^{-1}$; HRMS (GCMS/EI) $\mathrm{m} / z$ calcd for $\mathrm{C}_{12} \mathrm{H}_{12} \mathrm{NO}_{5}\left(\mathrm{M}-\mathrm{C}_{2} \mathrm{H}_{3} \mathrm{O}_{2}\right)^{+} 250.0715$, found 250.0721. Anal. Calcd for $\mathrm{C}_{14} \mathrm{H}_{15} \mathrm{NO}_{7}: \mathrm{C}, 54.37 ; \mathrm{H}, 4.89 ; \mathrm{N}, 4.53$. Found: C, 54.42; H, 5.05; N, 4.31 . 


\section{IIF. Synthesis of 4-Nitrotetrahydropyran-1-acetate (14f)}

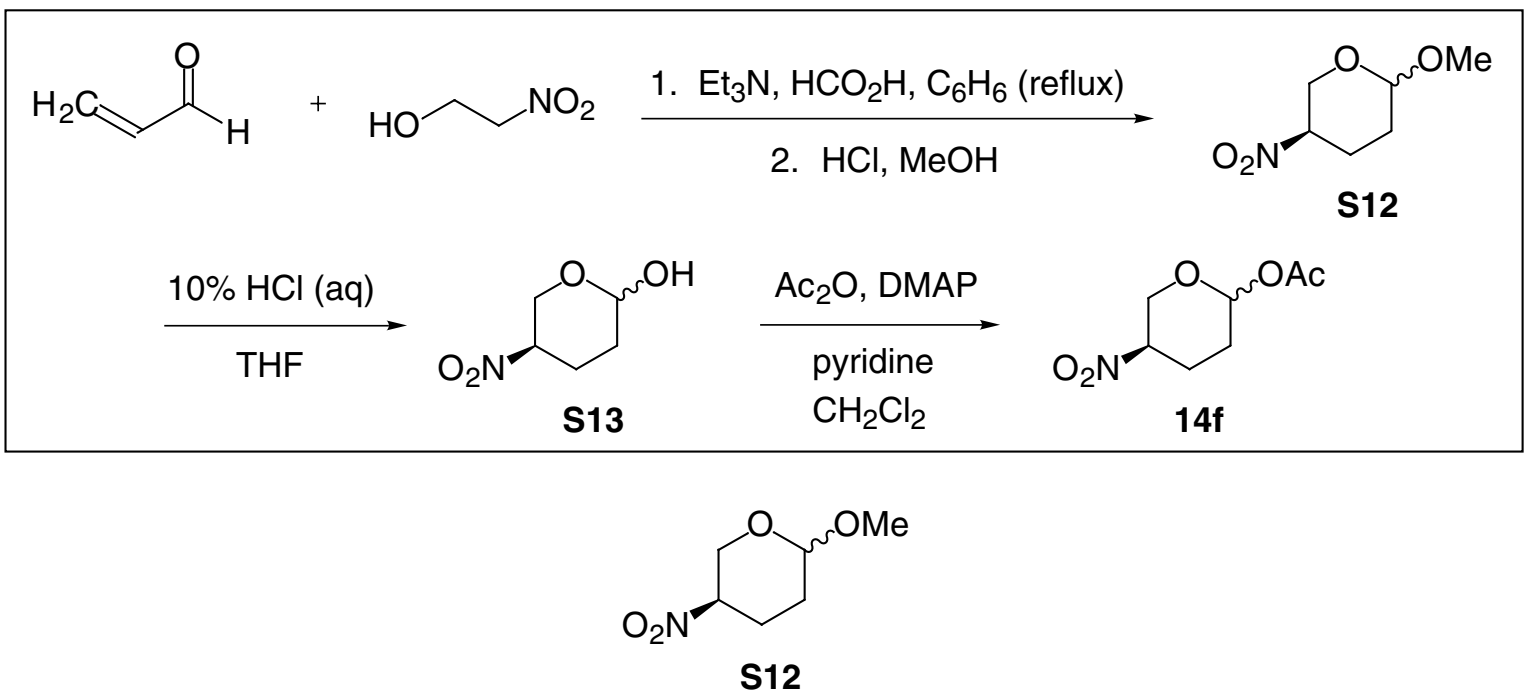

1-Methoxy-4-nitrotetrahydropyran (S12): ${ }^{4}$ A 1:1.75 mol mixture of triethylamine and formic acid $(0.150 \mathrm{~mL})$ was added to a solution of acrolein $(6.57 \mathrm{~mL}, 98.3 \mathrm{mmol}))$ and 2nitroethanol $(25.1 \mathrm{~g}, 275 \mathrm{mmol})$ in benzene $(50 \mathrm{~mL})$. The mixture was heated to reflux and stirred for $20 \mathrm{~h}$. The solvent was removed in vacuo, and the resulting brown oil was loaded on to a silica gel column packed with 9:1 hexanes/EtOAc. The reaction mixture was eluted (9:1 to 1:4 hexanes/EtOAc) to provide the product as a brown oil contaminated with inseparable byproducts. The oil was dissolved in methanol $(65 \mathrm{~mL})$. Concentrated $\mathrm{HCl}(0.650 \mathrm{~mL})$ was added, and the mixture was stirred at $50{ }^{\circ} \mathrm{C}$ for $24 \mathrm{~h}$. After neutralization with saturated aqueous $\mathrm{NaHCO}_{3}$, solvent was removed in vacuo. The remaining aqueous layer was extracted with $\mathrm{CH}_{2} \mathrm{Cl}_{2}(3 \times 50 \mathrm{~mL})$, and the combined organic layers were filtered through $\mathrm{Na}_{2} \mathrm{SO}_{4}$ and concentrated in vacuo. The resulting oil was purified by flash chromatography (1:9 to $3: 2$ $\mathrm{Et}_{2} \mathrm{O} /$ pentane) to afford the product (as a 1.1:1 mixture of anomers) as a colorless oil (7.38 $\mathrm{g}$, $47 \%):{ }^{3}{ }^{1} \mathrm{H}$ NMR $\left(500 \mathrm{MHz}, \mathrm{CDCl}_{3}\right) \delta 4.66(\mathrm{t}, J=2.8,1.1 \mathrm{H}), 4.62(\mathrm{t}, J=2.8,1 \mathrm{H}), 4.51(\mathrm{~m}$, $1 \mathrm{H}), 4.38$ (quint, $J=3.5,1.1 \mathrm{H}), 4.26(\mathrm{dt}, J=12.8,2.5,1.1 \mathrm{H}), 4.14(\mathrm{dd}, J=11.8,3.0,1.1 \mathrm{H})$, $4.11(\mathrm{t}, J=10.2,1 \mathrm{H}), 3.99(\mathrm{ddd}, J=11.1,4.5,1.8,1 \mathrm{H}), 3.40(\mathrm{~s}, 3.3 \mathrm{H}), 3.39(\mathrm{~s}, 3 \mathrm{H}), 2.44-2.36$ $(\mathrm{m}, 2.1 \mathrm{H}), 2.31-2.19(\mathrm{~m}, 2.1 \mathrm{H}), 2.02(\mathrm{~m}, 1.1 \mathrm{H}), 1.93(\mathrm{dtd}, J=13.9,4.5,2.6,1 \mathrm{H}) ;{ }^{13} \mathrm{C} \mathrm{NMR}$ 
$\left(125 \mathrm{MHz}, \mathrm{CDCl}_{3}\right) \delta 98.0,97.4,79.1,78.9,60.3,59.9,55.2,55.0,28.0,25.4,23.2,20.9$; IR (thin film) $2942,1549,1050,850 \mathrm{~cm}^{-1}$; HRMS (Cl/isobutane) $m / z$ calcd for $\mathrm{C}_{5} \mathrm{H}_{8} \mathrm{NO}_{3}(\mathrm{M}-$ $\left.\mathrm{CH}_{3} \mathrm{O}\right)^{+}$130.0504, found 130.0507. Anal. Calcd for $\mathrm{C}_{6} \mathrm{H}_{11} \mathrm{NO}_{4}$ : C, 44.72; $\mathrm{H}, 6.88 ; \mathrm{N}, 8.69$. Found: C, 44.89; H, 6.98; N, 8.71.

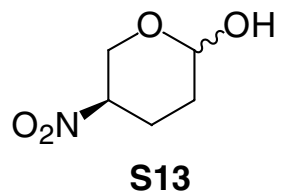

4-Nitrotetrahydropyran-1-ol (S13): An aqueous (10\%) solution of $\mathrm{HCl}(50 \mathrm{~mL})$ was added slowly to a solution of 1-methoxy-4-nitrotetrahydropyran (4.36 g, $27.1 \mathrm{mmol})$ in THF (100 $\mathrm{mL}$ ). After $24 \mathrm{~h}$, the mixture was neutralized with saturated aqueous $\mathrm{NaHCO}_{3}$. The solvent was removed in vacuo, and the remaining aqueous layer was extracted with $\mathrm{Et}_{2} \mathrm{O}(4 \times 100 \mathrm{~mL})$, dried over $\mathrm{MgSO}_{4}$, filtered, and concentrated in vacuo. The resulting white solid was purified by flash chromatography (1:9 to $3: 2 \mathrm{Et}_{2} \mathrm{O} /$ pentane) to afford the product (as a 3.8:1 mixture of anomers) as a white solid $(3.67 \mathrm{~g}, 92 \%):{ }^{3} \mathrm{mp} 98-100{ }^{\circ} \mathrm{C} ;{ }^{1} \mathrm{H}$ NMR $\left(500 \mathrm{MHz}, \mathrm{CDCl}_{3}\right) \delta 5.16(\mathrm{dd}, J=$ 7.1, 3.7, 3.8H), $5.08(\mathrm{dd}, J=7.5,4.3,1 \mathrm{H}), 4.48-4.41(\mathrm{~m}, 5.8 \mathrm{H}), 4.38(\mathrm{dd}, J=12.5,3.2,3.8 \mathrm{H})$, $4.20(\mathrm{dd}, J=12.5,4.0,3.8 \mathrm{H}), 3.98(\mathrm{~m}, 1 \mathrm{H}), 2.74(\mathrm{dd}, J=3.9,1.5,3.8 \mathrm{H}), 2.53(\mathrm{~m}, 1 \mathrm{H})$, 2.43-2.34 (m, 8.6H), $2.17(\mathrm{~m}, 1 \mathrm{H}), 2.05(\mathrm{~m}, 3.8 \mathrm{H}), 1.91(\mathrm{~m}, 1 \mathrm{H}), 1.83(\mathrm{~m}, 1 \mathrm{H}), 1.66$ (ddd, $J=$ 14.2, 9.0, 5.2, 3.8H) ${ }^{13} \mathrm{C}$ NMR $\left(125 \mathrm{MHz}, \mathrm{C}_{6} \mathrm{D}_{6}\right) \delta$ 93.6, 92.6, 79.4, 79.1, 63.5, 61.3, 28.9, 27.2, 23.8, 22.0; IR (KBr pellet) 3360, 2948, 1543, 1060, 964, $850 \mathrm{~cm}^{-1}$; HRMS (Cl/isobutene) $\mathrm{m} / z$ calcd for $\mathrm{C}_{5} \mathrm{H}_{8} \mathrm{NO}_{3}(\mathrm{M}-\mathrm{OH})^{+}$130.0504, found 130.0498. Anal. Calcd for $\mathrm{C}_{5} \mathrm{H}_{9} \mathrm{NO}_{4}$ : C, 40.82; H, 6.17; N, 9.52. Found: C, 41.02; H, 6.17; N, 9.54.

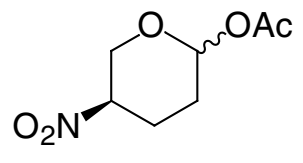

$14 f$

4-Nitrotetrahydropyran-1-acetate (14f): A solution of 4-nitrotetrahydropyran-1-ol (0.930 g, $6.32 \mathrm{mmol})$ in acetic anhydride $(8.0 \mathrm{~mL})$ was cooled to $0{ }^{\circ} \mathrm{C}$. Pyridine $(0.511 \mathrm{~mL}, 6.32 \mathrm{mmol})$ 
was added slowly, and the mixture was stirred at $0{ }^{\circ} \mathrm{C}$ for $3 \mathrm{~h}$ and poured into a separatory funnel containing saturated aqueous $\mathrm{NaHCO}_{3}(100 \mathrm{~mL})$ and pentane $(100 \mathrm{~mL})$. The layers were separated, and the aqueous layer was extracted with pentane $(2 \times 100 \mathrm{~mL})$. The combined organic layers were washed with $\mathrm{H}_{2} \mathrm{O}(100 \mathrm{~mL})$ and brine $(100 \mathrm{~mL})$, filtered through $\mathrm{Na}_{2} \mathrm{SO}_{4}$, and concentrated in vacuo. The resulting pale blue oil was purified by flash chromatography (1:9 to $2: 3 \mathrm{EtOAc/hexanes)} \mathrm{to} \mathrm{afford} \mathrm{the} \mathrm{product} \mathrm{(as} \mathrm{a} \mathrm{1.2:1} \mathrm{mixture} \mathrm{of} \mathrm{anomers)} \mathrm{as} \mathrm{a} \mathrm{colorless}$ oil $(0.486 \mathrm{~g}, 41 \%):{ }^{3} \mathrm{H} \mathrm{NMR}\left(500 \mathrm{MHz}, \mathrm{CDCl}_{3}\right) \delta 6.04(\mathrm{br} \mathrm{s}, 1 \mathrm{H}), 6.01(\mathrm{t}, J=2.9,1.2 \mathrm{H}), 4.56$ (m, 1.2H), $4.45(\mathrm{~m}, 1 \mathrm{H}), 4.41(\mathrm{~m}, 1 \mathrm{H}), 4.23(\mathrm{t}, J=2.9,1 \mathrm{H}), 4.19(\mathrm{~m}, 1.2 \mathrm{H}), 4.12(\mathrm{~m}, 1.2 \mathrm{H}), 2.53$ (m, 1H), $2.44(\mathrm{~m}, 1.2 \mathrm{H}), 2.35-2.25(\mathrm{~m}, 2.2 \mathrm{H}), 2.14(\mathrm{~s}, 3 \mathrm{H}), 2.13(\mathrm{~m}, 1 \mathrm{H}), 2.12(\mathrm{~s}, 3.6 \mathrm{H}), 2.00$ $(\mathrm{m}, 1.2 \mathrm{H}), 1.91(\mathrm{~m}, 1.2 \mathrm{H}), 1.73(\mathrm{ddd}, J=14.4,7.2,4.3,1 \mathrm{H}) ;{ }^{13} \mathrm{C} \mathrm{NMR}\left(125 \mathrm{MHz}, \mathrm{CDCl}_{3}\right) \delta$ $169.10,169.06,90.5,89.9,78.1,78.0,62.2,61.4,26.5,24.0,22.9,20.7,20.2$; IR (thin film) 2946, 1750, 1546, 1374, 1046, $935 \mathrm{~cm}^{-1}$; HRMS (CI/isobutene) $\mathrm{m} / z$ calcd for $\mathrm{C}_{5} \mathrm{H}_{8} \mathrm{NO}_{4}(\mathrm{M}-$ $\left.\mathrm{C}_{2} \mathrm{H}_{3} \mathrm{O}\right)^{+}$146.0453, found 146.0457. Anal. Calcd for $\mathrm{C}_{7} \mathrm{H}_{11} \mathrm{NO}_{5}: \mathrm{C}, 44.45 ; \mathrm{H}, 5.86 ; \mathrm{N}, 7.40$. Found: C, 44.26; H, 5.78; N, 7.34.

\section{IIG. Synthesis of 4-Azidotetrahydropyran-1-acetate (14g)}

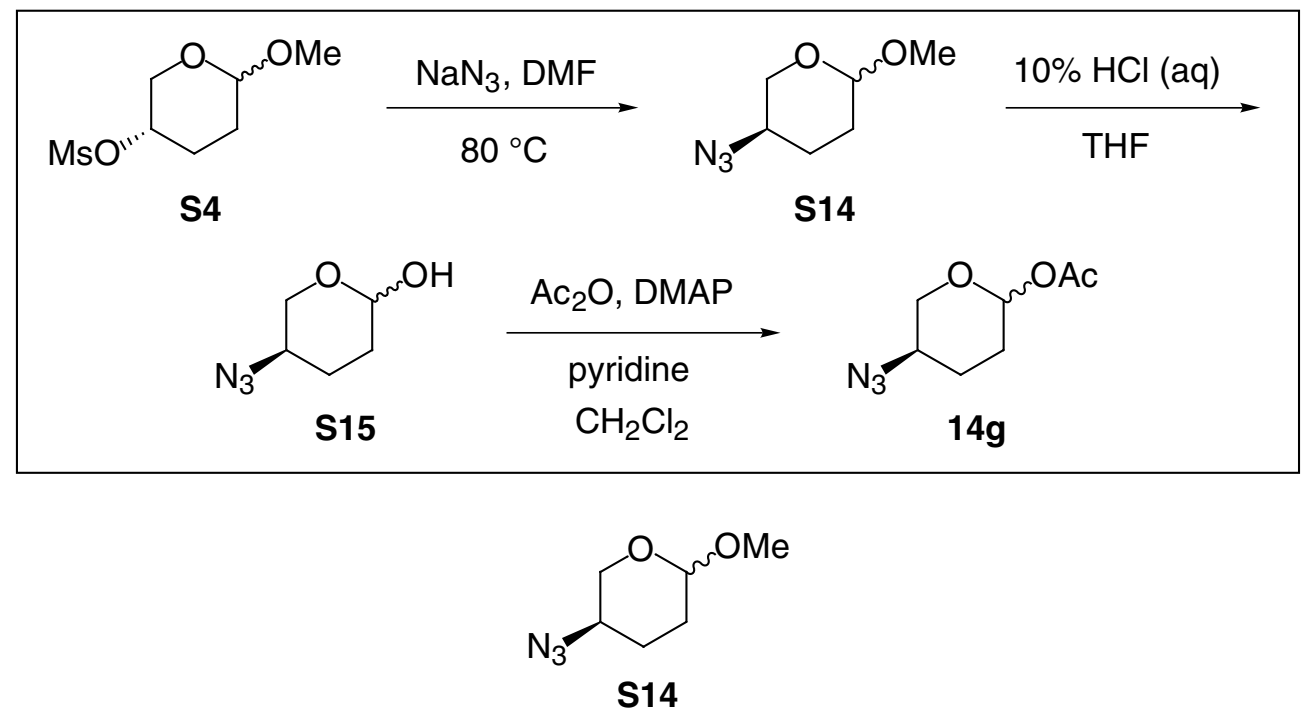

4-Azido-1-methoxytetrahydropyran (S14): Sodium azide (1.55 g, $23.8 \mathrm{mmol})$ was added to a solution of 4-methanesulfoxy-1-methoxytetrahydropyran in DMF (50 mL), and the mixture 
was stirred at $80{ }^{\circ} \mathrm{C}$ for $48 \mathrm{~h}$, cooled to $22^{\circ} \mathrm{C}$, and poured into $\mathrm{H}_{2} \mathrm{O}(300 \mathrm{~mL})$. The mixture was extracted with $\mathrm{Et}_{2} \mathrm{O}(4 \times 100 \mathrm{~mL})$, and the combined organic layers were dried over $\mathrm{MgSO}_{4}$, filtered, and concentrated in vacuo. The resulting oil was purified by flash chromatography (1:5 $\mathrm{Et}_{2} \mathrm{O} /$ pentane) to afford the product (as a 1.3:1 mixture of anomers) as a colorless oil $(0.720 \mathrm{~g}$, 95\%): ${ }^{1} \mathrm{H}$ NMR (500 MHz, $\left.\mathrm{CDCl}_{3}\right) \delta 4.61-4.58(\mathrm{~m}, 2.3 \mathrm{H}), 3.95(\mathrm{dd}, J=12.0,2.6,1 \mathrm{H}), 3.63$ $(\mathrm{ddd}, J=10.9,4.8,1.6,1.3 \mathrm{H}), 3.60-3.56(\mathrm{~m}, 2.3 \mathrm{H}), 3.52(\mathrm{ddd}, J=12.0,4.0,1.9,1 \mathrm{H}), 3.47(\mathrm{~m}$, 1.3H), $3.40(\mathrm{~s}, 3 \mathrm{H}), 3.37$ (s, 3.9H), $2.12(\mathrm{ddt}, J=13.5,11.2,4.0,1 \mathrm{H}), 2.00$ (dddd, $J=13.6,11.2$, 4.2, 3.2, 1H), 1.94-1.83 (m, 3.6H), 1.76-1.67 (m, 2.3H), 1.57 (m, 1.3H); ${ }^{13} \mathrm{C}$ NMR (125 MHz, $\left.\mathrm{CDCl}_{3}\right) \delta 98.7,97.3,62.1,61.9,55.6,55.5,55.2,54.8,28.4,25.9,23.9,23.1 ; \mathrm{IR}$ (thin film) 2938, 2101, 1257, $1130 \mathrm{~cm}^{-1}$; HRMS (CI/isobutane) $m / z$ calcd for $\mathrm{C}_{6} \mathrm{H}_{10} \mathrm{~N}_{3} \mathrm{O}_{2}(\mathrm{M}-\mathrm{H})^{+} 156.0773$, found 156.0774 .

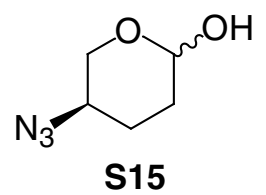

4-Azidotetrahydropyran-1-ol (S15): An aqueous (10\%) solution of $\mathrm{HCl}(10 \mathrm{~mL})$ was added slowly to a solution of 1-azido-1-methoxytetrahydropyran (0.496 g, $3.16 \mathrm{mmol})$ in THF (20 mL). After $24 \mathrm{~h}$, the mixture was neutralized with saturated aqueous $\mathrm{NaHCO}_{3}$. The solvent was removed in vacuo, and the remaining aqueous layer was extracted with $\mathrm{Et}_{2} \mathrm{O}(4 \times 50 \mathrm{~mL})$, dried over $\mathrm{MgSO}_{4}$, filtered, and concentrated in vacuo. The resulting oil was purified by flash chromatography (1:4 to $1: 2 \mathrm{Et}_{2} \mathrm{O}$ /pentane) to afford the product (as a 1:1 mixture of anomers) as a colorless oil $(0.428 \mathrm{~g}, 95 \%):{ }^{3} \mathrm{H}$ NMR $\left(500 \mathrm{MHz}, \mathrm{CDCl}_{3}\right) \delta 5.07-5.02(\mathrm{~m}, 2 \mathrm{H}), 4.12(\mathrm{ddd}, J$ $=11.8,3.1,0.9,1 \mathrm{H}), 3.87(\mathrm{dd}, J=11.4,8.0,1 \mathrm{H}), 3.67(\mathrm{ddd}, J=11.4,3.9,1.4,1 \mathrm{H}), 3.58(\mathrm{ddd}, J$ $=13.1,6.7,3.6,1 \mathrm{H}), 3.51-3.46(\mathrm{~m}, 2 \mathrm{H}), 3.03(\mathrm{br} \mathrm{s}, 1 \mathrm{H}), 2.95(\mathrm{br} \mathrm{s}, 1 \mathrm{H}), 2.18(\mathrm{~m}, 1 \mathrm{H})$, 2.06-1.81 (m, 4H), 1.80-1.65 (m, 2H), $1.58(\mathrm{~m}, 1 \mathrm{H}) ;{ }^{13} \mathrm{C}$ NMR (125 MHz, $\left.\mathrm{CDCl}_{3}\right) \delta 93.1$, 92.1, 63.8, 63.4, 55.3, 55.2, 28.6, 27.6, 24.2, 24.0; IR (thin film) 3399, 2951, 2103, $1259 \mathrm{~cm}^{-1}$; 
HRMS (Cl/isobutane) $m / z$ calcd for $\mathrm{C}_{5} \mathrm{H}_{8} \mathrm{~N}_{3} \mathrm{O}(\mathrm{M}-\mathrm{OH})^{+}$126.0667, found 126.0671. Anal. Calcd for $\mathrm{C}_{5} \mathrm{H}_{9} \mathrm{~N}_{3} \mathrm{O}_{2} \mathrm{C}, 41.95 ; \mathrm{H}, 6.34$. Found: $\mathrm{C}, 41.52 ; \mathrm{H}, 6.39$.

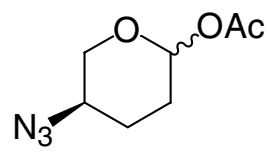

$14 \mathrm{~g}$

4-Azidotetrahydropyran-1-acetate (14g): A solution of 4-azidotetrahydropyra-1-ol (0.193 $\mathrm{g}, 1.35 \mathrm{mmol})$, acetic anhydride $(0.636 \mathrm{~mL}, 6.74 \mathrm{mmol})$, pyridine $(0.436 \mathrm{~mL}, 5.39 \mathrm{mmol})$ and 4-dimethylaminopyridine $(0.199 \mathrm{~g}, 1.62 \mathrm{mmol})$ in $\mathrm{CH}_{2} \mathrm{Cl}_{2}(20 \mathrm{~mL})$ was stirred for $24 \mathrm{~h}$. Saturated aqueous $\mathrm{NH}_{4} \mathrm{Cl}(10 \mathrm{~mL})$ was added, and $\mathrm{CH}_{2} \mathrm{Cl}_{2}$ was removed in vacuo. The residue was dissolved in MTBE $(75 \mathrm{~mL})$, and the layers were separated. The organic layer was washed with saturated aqueous $\mathrm{Na}_{2} \mathrm{HPO}_{4}(4 \times 20 \mathrm{~mL}), \mathrm{NaH}_{2} \mathrm{PO}_{4}(4 \times 20 \mathrm{~mL})$, and $\mathrm{CuSO}_{4}(5 \times 20 \mathrm{~mL})$, filtered through $\mathrm{Na}_{2} \mathrm{SO}_{4}$, and concentrated in vacuo. The resulting oil was purified by flash chromatography (1:4 $\mathrm{Et}_{2} \mathrm{O} /$ pentane) to afford the product (as a 1:1 mixture of anomers) as a colorless oil $(0.239 \mathrm{~g}, 96 \%):{ }^{3} \mathrm{H}$ NMR $\left(500 \mathrm{MHz}, \mathrm{CDCl}_{3}\right) \delta 6.02-5.99(\mathrm{~m}, 2 \mathrm{H}), 4.02(\mathrm{dd}, J=$ 13.3, 3.6, 1H), 3.78 (ddd, $J=11.0,4.4,1.9,1 \mathrm{H}), 3.70-3.62(\mathrm{~m}, 3 \mathrm{H}), 3.55(\mathrm{~m}, 1 \mathrm{H}), 2.18-2.06$ (m, 8H), $2.02(\mathrm{~m}, 1 \mathrm{H}), 1.94-1.79(\mathrm{~m}, 4 \mathrm{H}), 1.65(\mathrm{~m}, 1 \mathrm{H}) ;{ }^{13} \mathrm{C} \mathrm{NMR}\left(125 \mathrm{MHz}, \mathrm{CDCl}_{3}\right) \delta 169.5$, 169.4, 91.5, 90.3, 63.9, 63.8, 55.1, 54.9, 27.3, 24.5, 23.9, 22.8, 21.0; IR (thin film) 2944, 2105, 1748, 1242, $1209 \mathrm{~cm}^{-1}$; HRMS (CI/isobutane) $\mathrm{m} / z$ calcd for $\mathrm{C}_{5} \mathrm{H}_{8} \mathrm{~N}_{3} \mathrm{O}\left(\mathrm{M}-\mathrm{C}_{2} \mathrm{H}_{3} \mathrm{O}_{2}\right)^{+}$ 126.0667, found 126.0664. Anal. Calcd for $\mathrm{C}_{7} \mathrm{H}_{11} \mathrm{~N}_{3} \mathrm{O}_{3}$ : C, 45.40; H, 5.99. Found: C, 45.67; H, 6.16 . 
IIH. Synthesis of 4-Fluorotetrahydropyran-1-acetate (21a)

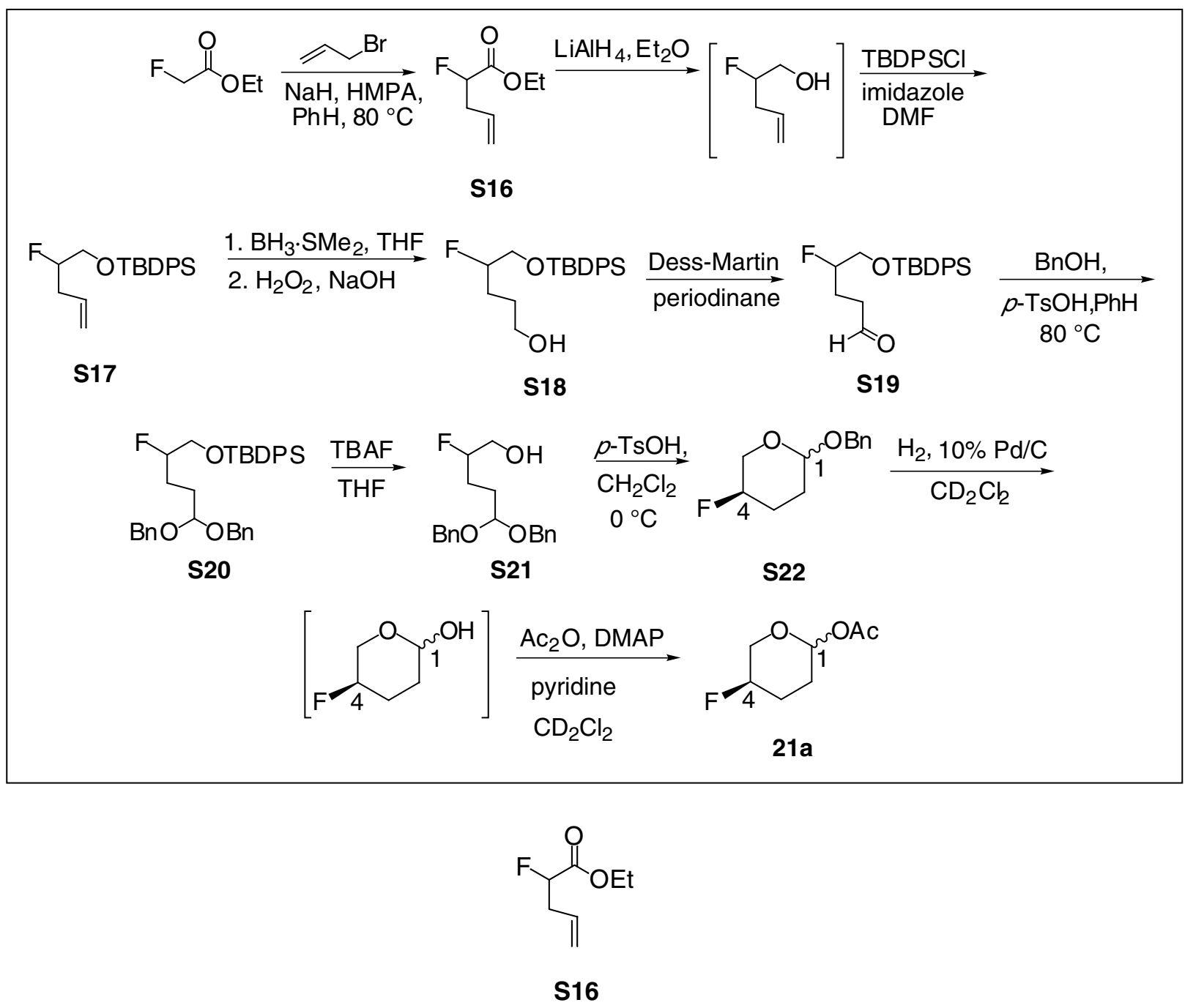

Ethyl-2-fluoro-4-pentenoate (S16): $:^{5}$ Allyl bromide $(57.01 \mathrm{~g}, 471.3 \mathrm{mmol})$ was added to a solution of ethyl fluoroacetate $(50.00 \mathrm{~g}, 471.3 \mathrm{mmol})$ in benzene and HMPA (236 mL and 118 $\mathrm{mL})$ respectively. Sodium hydride $(11.31 \mathrm{~g}, 471.3 \mathrm{mmol})$ was washed with hexanes $(3 \times 150$ $\mathrm{mL}$ ) and added to the solution. The reaction mixture was stirred and heated at $80{ }^{\circ} \mathrm{C}$ for $12 \mathrm{~h}$. The reaction mixture was cooled to $23{ }^{\circ} \mathrm{C}$ and $\mathrm{H}_{2} \mathrm{O}$ was added $(350 \mathrm{~mL})$, and the layers were separated. The organic layer was washed with $\mathrm{H}_{2} \mathrm{O}(3 \times 150 \mathrm{~mL})$, and the aqueous layer was neutralized with $0.1 \mathrm{M} \mathrm{H}_{2} \mathrm{SO}_{4}$. The aqueous layer was extracted with $\mathrm{Et}_{2} \mathrm{O}(3 \times 240 \mathrm{~mL})$. The combined organic layers were washed with saturated aqueous $\mathrm{NaCl}(350 \mathrm{~mL})$, dried over 
$\mathrm{MgSO}_{4}$, filtered, and concentrated in vacuo. The resulting yellow oil was purified by flash column chromatography (pentane to $1: 9 \mathrm{Et}_{2} \mathrm{O} /$ pentane) to afford the product as a colorless oil (33.39 g, 48\%): ${ }^{1} \mathrm{H}$ NMR (500 MHz, $\left.\mathrm{CDCl}_{3}\right) \delta 5.87-5.78(\mathrm{~m}, 1 \mathrm{H}), 5.22-5.17(\mathrm{~m}, 2 \mathrm{H})$, 5.01-4.89 (ddd, $J=48.7,6.9,4.6,1 \mathrm{H}), 4.26(\mathrm{q}, J=7.1,2 \mathrm{H}), 2.68-2.61(\mathrm{~m}, 2 \mathrm{H}), 1.31$ (t, $J=7.1$, $3 \mathrm{H}) ;{ }^{13} \mathrm{C}$ NMR $\left(125 \mathrm{MHz}, \mathrm{CDCl}_{3}\right) \delta 169.8(\mathrm{~d}, J=24), 131.41(\mathrm{~d}, J=4), 119.7,89.3(\mathrm{~d}, J=$ 185), 61.9, $37.2(\mathrm{~d}, J=21), 14.6 ;{ }^{19} \mathrm{~F}$ NMR (376 MHz, $\mathrm{CDCl}_{3}$ ) $\delta-191.6(\mathrm{~m})$; IR (thin film) 2978, 1756, 1278, 1211, 1072, $750 \mathrm{~cm}^{-1}$; HRMS (EI/GCMS) $m / z$ calcd for $\mathrm{C}_{7} \mathrm{H}_{11} \mathrm{FO}_{2}(\mathrm{M})^{+}$ 146.0743, found 146.0745 .

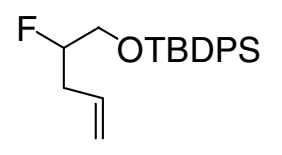

S17

1-(tert-Butyldiphenylsilyloxy)-2-fluoropent-4-en (S17): A suspension of $\mathrm{LiAlH}_{4}(6.08 \mathrm{~g}$, $160 \mathrm{mmol})$ in $\mathrm{Et}_{2} \mathrm{O}(1033 \mathrm{~mL})$ was cooled to $0{ }^{\circ} \mathrm{C}$, and a solution of ethyl-2-fluoro-4-pentenoate $(15.71 \mathrm{~g}, 80.08 \mathrm{mmol})$ in $\mathrm{Et}_{2} \mathrm{O}(235 \mathrm{~mL})$ was added dropwise. The reaction mixture was warmed to $23{ }^{\circ} \mathrm{C}$ and stirred for $18 \mathrm{~h}$ before dropwise addition of $\mathrm{H}_{2} \mathrm{O}(8.40 \mathrm{~mL}), 15 \% \mathrm{NaOH}(8.40 \mathrm{~mL})$, and $\mathrm{H}_{2} \mathrm{O}(25.0 \mathrm{~mL})$. Anhydrous $\mathrm{Na}_{2} \mathrm{SO}_{4}(15.7 \mathrm{~g})$ was added, and the mixture was filtered, dried over $\mathrm{MgSO}_{4}$, filtered, and concentrated in vacuo to afford a yellow oil. Because of the volatility of the alcohol, the impure oil was submitted to silylation conditions. To a solution of 2-fluoro-4penten-1-ol in DMF (94 mL) were added tert-butyldiphenylsilyl chloride (24.2 g, $88.1 \mathrm{mmol})$ and imidazole $(12.0 \mathrm{~g}, 176 \mathrm{mmol})$. After $12 \mathrm{~h}$, the mixture was diluted with $\mathrm{Et}_{2} \mathrm{O}(650 \mathrm{~mL})$ and washed with $\mathrm{H}_{2} \mathrm{O}(200 \mathrm{~mL})$. The layers were separated, and the aqueous layer was extracted with $\mathrm{Et}_{2} \mathrm{O}(3 \times 250 \mathrm{~mL})$, dried over $\mathrm{MgSO}_{4}$, filtered and concentrated in vacuo. The resulting yellow oil was purified by flash column chromatography (pentane to $1: 9 \mathrm{Et}_{2} \mathrm{O}$ /pentane) to afford the desired product as a colorless oil $\left(13.17 \mathrm{~g}, 48 \%\right.$ over two steps): ${ }^{1} \mathrm{H}$ NMR $\left(500 \mathrm{MHz}, \mathrm{CDCl}_{3}\right) \delta$ 7.69-7.67 (m, 4H), 7.43-7.36 (m, 6H), $5.78(\mathrm{~m}, 1 \mathrm{H}), 5.14-5.10(\mathrm{~m}, 1 \mathrm{H}), 5.08(\mathrm{~m}, 1 \mathrm{H}), 4.63(\mathrm{~m}$, 1H), 3.78-3.74 (m, 2H), 2.49-2.43 (m, 2H), $1.07(\mathrm{~s}, 9 \mathrm{H}) ;{ }^{13} \mathrm{C}$ NMR (125 MHz, $\left.\mathrm{CDCl}_{3}\right)$ 
$\delta 136.14,136.12,133.80,133.79,133.5,133.4,130.3,128.3,94.3(\mathrm{~d}, \quad J=174), 65.6(\mathrm{~d}, \quad J=$ 24), $36.3(\mathrm{~d}, J=21), 27.3(\mathrm{~d}, J=14), 19.8 ;{ }^{19} \mathrm{~F} \mathrm{NMR}\left(376 \mathrm{MHz}, \mathrm{CDCl}_{3}\right) \delta-186.4(\mathrm{dq}, J=51.2$, 23.3); IR (thin film) 3067, 2922, 1422, 1111, 756, $700 \mathrm{~cm}^{-1}$; HRMS (EI/GCMS) $\mathrm{m} / z$ calcd for $\mathrm{C}_{17} \mathrm{H}_{18} \mathrm{FOSi}\left(\mathrm{M}-\mathrm{C}_{4} \mathrm{H}_{9}\right)^{+}$285.1111, found 285.1113. Anal. Calcd for $\mathrm{C}_{21} \mathrm{H}_{27} \mathrm{FOSi}$ : C, 73.64; $\mathrm{H}$, 7.95. Found: C, 74.01; H, 8.37.

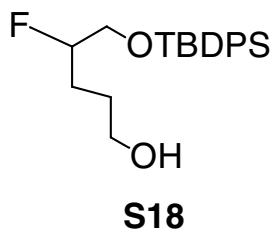

1-(tert-Butyldiphenylsilyloxy)-2-fluoropentan-5-ol (S18): To a solution of 1-(tertbutyldiphenylsilyloxy)-2-fluoropent-4-ene $(9.00 \mathrm{~g}, 26.3 \mathrm{mmol})$ in THF $(22 \mathrm{~mL})$ at $0{ }^{\circ} \mathrm{C}$ was added $10 \mathrm{M} \mathrm{BH}_{3} \cdot \mathrm{SMe}_{2}(1.1 \mathrm{~mL})$. The reaction mixture was allowed to warm to $23{ }^{\circ} \mathrm{C}$ and stirred for $72 \mathrm{~h}$. The reaction mixture was cooled to $0{ }^{\circ} \mathrm{C}$ and $10 \% \mathrm{NaOH}(31 \mathrm{~mL})$ followed by $30 \% \mathrm{H}_{2} \mathrm{O}_{2}(15 \mathrm{~mL})$ were added. The reaction mixture was allowed to warm to $23{ }^{\circ} \mathrm{C}$ and stirred for $48 \mathrm{~h}$. Solid $\mathrm{K}_{2} \mathrm{CO}_{3}(45.6 \mathrm{~g})$ was added and the mixture was stirred for an additional $2 \mathrm{~h}$. The layers were separated, and the aqueous layer was extracted with $\mathrm{Et}_{2} \mathrm{O}(3 \times 50 \mathrm{~mL})$. The combined organic phases were dried over $\mathrm{MgSO}_{4}$, filtered and concentrated in vacuo. The resulting yellow oil was purified by flash column chromatography $\left(1: 9 \mathrm{Et}_{2} \mathrm{O} /\right.$ pentane to $2: 3$ $\mathrm{Et}_{2} \mathrm{O} /$ pentane) to afford a colorless oil $(7.86 \mathrm{~g}, 83 \%):{ }^{1} \mathrm{H} \mathrm{NMR}\left(500 \mathrm{MHz}, \mathrm{CDCl}_{3}\right) \delta 7.68(\mathrm{~m}$, 4H), 7.41-7.35 (m, 6H), 4.64-4.51 (m, 1H), 3.78-3.72 (dd, $J=22.6,4.5,2 \mathrm{H}), 3.60(\mathrm{~m}, 2 \mathrm{H})$, 2.22 (bs, $1 \mathrm{H}), \quad 1.75-1.58 \quad(\mathrm{~m}, 4 \mathrm{H}), 1.07 \quad(\mathrm{~s}, 9 \mathrm{H}) ;{ }^{13} \mathrm{C} \quad \mathrm{NMR}\left(125 \mathrm{MHz}, \mathrm{CDCl}_{3}\right)$ $\delta 135.9,133.60,133.57,130.1,128.1,95.0(\mathrm{~d}, J=170), 66.1(\mathrm{~d}, J=24), 62.6,28.4(\mathrm{~d}, J=$ 4), $27.8(\mathrm{~d}, J=21), 27.1,19.6 ;{ }^{19} \mathrm{~F}$ NMR (376 MHz, $\left.\mathrm{CDCl}_{3}\right) \delta-186.8(\mathrm{~m})$; IR (thin film) 3389 , 2944, 1428, 1113, 758, $703 \mathrm{~cm}^{-1}$; HRMS (EI/GCMS) $m / z$ calcd for $\mathrm{C}_{17} \mathrm{H}_{20} \mathrm{FO}_{2} \mathrm{Si}\left(\mathrm{M}-\mathrm{C}_{4} \mathrm{H}_{9}\right)^{+}$ 303.1217, found 303.1220. Anal. Calcd for $\mathrm{C}_{21} \mathrm{H}_{29} \mathrm{FO}_{2} \mathrm{Si}$ : C, 69.96; H, 8.11. Found: C, 69.90; $\mathrm{H}, 8.23$. 


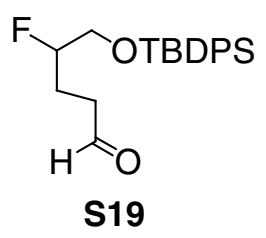

5-(tert-Butyldiphenylsilyloxy)-4-fluoropentanal (S19): Dess-Martin periodinane ${ }^{6,7}(0.828$ $\mathrm{g}, 1.95 \mathrm{mmol}$ ) was added to a solution of 1-(tert-butyldiphenylsilyloxy)-2-fluoropentan-5-ol (0.502g, $1.39 \mathrm{mmol})$ in $\mathrm{CH}_{2} \mathrm{Cl}_{2}(13 \mathrm{~mL})$. The mixture was stirred for $16 \mathrm{~h}$. A saturated aqueous solution of $\mathrm{NaHCO}_{3}$ and $\mathrm{Na}_{2} \mathrm{~S}_{2} \mathrm{O}_{3}(30 \mathrm{~mL})$ was added. After stirring for $15 \mathrm{~min}$, the layers were separated and the aqueous layer was extracted with $\mathrm{CH}_{2} \mathrm{Cl}_{2}(3 \times 60 \mathrm{~mL})$. The combined organic phases were washed with saturated aqueous $\mathrm{NaHCO}_{3}(30 \mathrm{~mL})$, dried over $\mathrm{Na}_{2} \mathrm{SO}_{4}$, and concentrated in vacuo. The resulting oil was purified by flash column chromatography (pentane to $1: 4 \mathrm{Et}_{2} \mathrm{O} /$ pentane) to afford the product as a colorless oil $(0.473 \mathrm{~g}, 95 \%):{ }^{1} \mathrm{H} \mathrm{NMR}(500 \mathrm{MHz}$, $\left.\mathrm{CDCl}_{3}\right) \delta 9.75(\mathrm{~s}, 1 \mathrm{H}), 7.67(\mathrm{~m}, 4 \mathrm{H}), 7.42-7.36(\mathrm{~m}, 6 \mathrm{H}), 4.65-4.50(\mathrm{~m}, 1 \mathrm{H}), 3.79-3.73(\mathrm{~m}, 2 \mathrm{H})$, $2.57(\mathrm{~m}, \quad 2 \mathrm{H}), \quad 2.00 \quad(\mathrm{~m}, \quad 2 \mathrm{H}), \quad 1.07 \quad(\mathrm{~s}, \quad 9 \mathrm{H}) ; \quad{ }^{13} \mathrm{C} \quad \mathrm{NMR} \quad\left(125 \mathrm{MHz}, \quad \mathrm{CDCl}_{3}\right)$ $\delta 201.8,136.11,136.09,133.62,133.60,130.4,128.3,94.0(\mathrm{~d}, \quad J=173), 65.9(\mathrm{~d}, \quad J=$ 24), $39.8(\mathrm{~d}, J=4), 27.3,24.2(\mathrm{~d}, J=21), 19.8 ;{ }^{19} \mathrm{~F}$ NMR (376 MHz, $\left.\mathrm{CDCl}_{3}\right) \delta-189.0(\mathrm{~m}) ; \mathrm{IR}$ (thin film) 2932, 1726, 1428, 1113, 911, $735 \mathrm{~cm}^{-1}$; HRMS (EI/GCMS) $m / z$ calcd for $\mathrm{C}_{17} \mathrm{H}_{18} \mathrm{FO}_{2} \mathrm{Si}\left(\mathrm{M}-\mathrm{C}_{4} \mathrm{H}_{9}\right)^{+}$301.1060, found 301.1062. Anal. Calcd for $\mathrm{C}_{21} \mathrm{H}_{27} \mathrm{FO}_{2} \mathrm{Si}: \mathrm{C}, 70.35$; H, 7.59. Found: C, 70.28; H, 7.59.

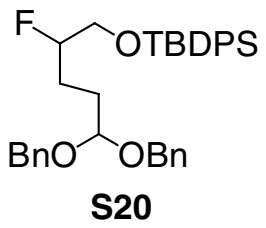

5-(tert-Butyl(diphenylsilyloxy)-4-fluoropent-dibenzylacetal $(\mathbf{S 2 0}):^{8}$ A solution of 5(tert-butyldiphenylsilyloxy)-4-fluoropentanal $(0.672 \mathrm{~g}, 1.87 \mathrm{mmol})$, benzyl alcohol (0.446 g, $4.12 \mathrm{mmol})$, and $p$-toluenesulfonic acid monohydrate $(0.004 \mathrm{~g}, 0.019 \mathrm{mmol})$ in benzene $(24 \mathrm{~mL})$ 
was heated to $80^{\circ} \mathrm{C}$ for $20 \mathrm{~h}$ employing a Dean Stark trap. The reaction mixture was cooled to $23{ }^{\circ} \mathrm{C}$, washed with saturated aqueous $\mathrm{NaHCO}_{3}(3 \times 10 \mathrm{~mL})$, dried over $\mathrm{MgSO}_{4}$, filtered and concentrated in vacuo. The resulting oil was purified by flash column chromatography (pentane to $1: 4 \mathrm{Et}_{2} \mathrm{O} /$ pentane) to afford the product as a colorless oil $(1.38 \mathrm{~g}, 86 \%):{ }^{1} \mathrm{H} \mathrm{NMR}(500 \mathrm{MHz}$, $\left.\mathrm{CDCl}_{3}\right) \delta 7.67(\mathrm{~m}, 4 \mathrm{H}), 7.42-7.27(\mathrm{~m}, 16 \mathrm{H}), 4.75(\mathrm{t}, J=5.4,1 \mathrm{H}), 4.67(\mathrm{~d}, J=5.0,1 \mathrm{H}), 4.64(\mathrm{~d}$, $J=4.9,1 \mathrm{H}), 4.55(\mathrm{~m}, 3 \mathrm{H}), 3.73(\mathrm{~m}, 2 \mathrm{H}), 1.93-1.75(\mathrm{~m}, 4 \mathrm{H}), 1.06(\mathrm{~s}, 9 \mathrm{H}) ;{ }^{13} \mathrm{C}$ NMR $(125 \mathrm{MHz}$, $\left.\mathrm{CDCl}_{3}\right) \delta 138.6,136.1,133.8,130.2,129.1,128.93,128.90,128.3,128.2,128.12,128.08$, 102.3, 94.9 (d, $J=170), 68.0$ (d, $J=26), 66.2(\mathrm{~d}, J=24), 29.3(\mathrm{~d}, J=4), 27.3,27.1(\mathrm{~d}, J=$ 21), 19.8; ${ }^{19} \mathrm{~F} \mathrm{NMR} \mathrm{(376} \mathrm{MHz,} \mathrm{CDCl}_{3}$ ) $\delta-187.6$ (m); IR (thin film) 3067, 2922, 1422, 1111, 1022, $733 \mathrm{~cm}^{-1}$; HRMS (EI/GCMS) $m / z$ calcd for $\mathrm{C}_{35} \mathrm{H}_{41} \mathrm{FO}_{3} \mathrm{SiNa}(\mathrm{M}+\mathrm{Na})^{+}$579.2707, found 579.2709. Anal. Calcd for $\mathrm{C}_{35} \mathrm{H}_{41} \mathrm{FO}_{3} \mathrm{Si}$ : C, 75.50; H, 7.42. Found: C, 75.50; H, 7.44.

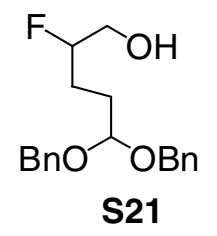

4-Fluoro-1,1-dibenzyloxy-5-pentanol (S21): Tetrabutylammonium fluoride was added as a $1 \mathrm{M}$ solution in THF (1.4 mL) to a solution of 5-(tert-butyldiphenylsilyloxy)-4-fluoropent-dibenzylacetal $(0.638 \mathrm{~g}, 1.15 \mathrm{mmol})$ in THF $(13 \mathrm{~mL})$. The mixture was allowed to stir for $20 \mathrm{~h}$ at $23{ }^{\circ} \mathrm{C}$. Saturated aqueous $\mathrm{NH}_{4} \mathrm{Cl}(4 \mathrm{~mL})$ was added, and the layers were separated. The aqueous layer was extracted with $\mathrm{Et}_{2} \mathrm{O}(3 \times 10 \mathrm{~mL})$. The combined organic layers were washed with saturated aqueous $\mathrm{NaCl}(4$ $\mathrm{mL}$ ), dried over $\mathrm{MgSO}_{4}$, filtered and concentrated in vacuo. The resulting oil was purified by flash column chromatography (1:8 Et ${ }_{2} \mathrm{O} /$ pentane to $1: 1 \mathrm{Et}_{2} \mathrm{O} /$ pentane $)$ to afford the product as a colorless oil (0.314 g, 86\%): ${ }^{1} \mathrm{H}$ NMR (500 MHz, $\left.\mathrm{CDCl}_{3}\right) \delta 7.32-7.25(\mathrm{~m}, 10 \mathrm{H}), 4.73(\mathrm{t}, J=5.5,1 \mathrm{H}), 4.64$ (dd, $J=11.7,3.8,4 \mathrm{H}), 4.53(\mathrm{~d}, J=11.7,1 \mathrm{H}), 3.61-3.54(\mathrm{~m}, 2 \mathrm{H}), 2.63$ (bs, $1 \mathrm{H}), 1.94-1.89(\mathrm{~m}, 1 \mathrm{H})$, $\begin{array}{lllllll}1.83-1.71 & (\mathrm{~m}, & 3 \mathrm{H}) ; & { }^{13} \mathrm{C} & \mathrm{NMR} & (125 \quad \mathrm{MHz}, & \left.\mathrm{CDCl}_{3}\right)\end{array}$ $\delta 138.8,138.6,129.00,128.97,128.9,128.8,128.6,128.5,128.4,128.3,128.2,128.12,128.05$, 
102.2, $95.5(\mathrm{~d}, J=169), 68.2(\mathrm{~d}, J=23), 67.8,65.2(\mathrm{~d}, J=22), 29.3(\mathrm{~d}, J=4), 26.7(\mathrm{~d}, J=21) ;{ }^{19} \mathrm{~F}$ $\operatorname{NMR}\left(376 \mathrm{MHz}, \mathrm{CDCl}_{3}\right) \delta-189.5$ (m); IR (thin film) 3426, 2931, 1454, 1121, 1044, 1025, $754 \mathrm{~cm}^{-1}$; HRMS (EI/GCMS) $m / z$ calcd for $\mathrm{C}_{12} \mathrm{H}_{16} \mathrm{FO}_{3}\left(\mathrm{M}-\mathrm{C}_{7} \mathrm{H}_{7}\right)^{+} 227.1083$, found 227.1075. Anal. Calcd for $\mathrm{C}_{19} \mathrm{H}_{23} \mathrm{FO}_{3}$ : $\mathrm{C}, 71.68 ; \mathrm{H}, 7.28$. Found: $\mathrm{C}, 71.38 ; \mathrm{H}, 7.41$.

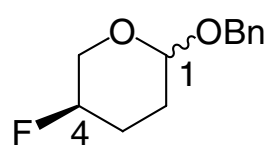

S22

4-Fluoro-1-benzyloxytetrahydropyran (S22): ${ }^{9} \quad p$-Toluenesulfonic acid monohydrate $(0.005 \mathrm{~g}, 0.010 \mathrm{mmol})$ was added to a solution of 4-fluoro-1,1-dibenzyloxy-5-pentanol $(0.314 \mathrm{~g}$, $0.986 \mathrm{mmol})$ in $\mathrm{CH}_{2} \mathrm{Cl}_{2}(1.2 \mathrm{~mL})$ at $0{ }^{\circ} \mathrm{C}$. After $17 \mathrm{~h}$, the solution was concentrated in vacuo to afford a yellow oil. The unpurified product was purified by flash column chromatography (1:9 $\mathrm{Et}_{2} \mathrm{O} /$ pentane to $4: 7 \mathrm{Et}_{2} \mathrm{O}$ /pentane) to afford the product (as a 3:5 mixture of isomers) as a colorless oil $(0.168 \mathrm{~g}, 81 \%)$ : ${ }^{1} \mathrm{H}$ NMR $\left(500 \mathrm{MHz}, \mathrm{CDCl}_{3}\right) \delta$ 7.36-7.25 (m, 10H), 4.91 (bs, $1 \mathrm{H}), 4.78(\mathrm{~d}, J=12.0,1 \mathrm{H}), 4.73(\mathrm{~d}, J=12.0,1 \mathrm{H}), 4.69(\mathrm{~m}, 1 \mathrm{H}), 4.59-4.50(\mathrm{~m}, 4 \mathrm{H}), 3.95-3.74$ (m, 4H), 2.13-2.07 (m, 3H), 1.93-1.90 (m, 3H), $1.75(\mathrm{~m}, 1 \mathrm{H}), 1.65(\mathrm{~m}, 1 \mathrm{H}) ;{ }^{13} \mathrm{C}$ NMR $(125$ $\left.\mathrm{MHz}, \mathrm{CDCl}_{3}\right) \delta 138.4,138.2,128.9,128.3,128.23,128.16,128.1,96.9,96.3,87.3(\mathrm{~d}, \quad J=$ 48), 85.9 (d, 49), 69.5, 69.4, 64.0 (d, $J=25), 62.6$ (d, $J=20), 28.2$ (d, $J=6$ ), 26.4 (d, $J=$ 20), $24.9(\mathrm{~d}, J=1), 23.4(\mathrm{~d}, J=21) ;{ }^{19} \mathrm{~F}$ NMR $\left(376 \mathrm{MHz}, \mathrm{CDCl}_{3}\right) \delta-186.9(\mathrm{~m}),-189.5(\mathrm{~m})$; IR (thin film) 2933, 1450, 1367, 1211, 1128, 1078, 1033, 978, 911, $728 \mathrm{~cm}^{-1}$; HRMS (EI/GCMS) $m / z$ calcd for $\mathrm{C}_{12} \mathrm{H}_{15} \mathrm{FO}_{2}(\mathrm{M})^{+}$210.1056, found 210.1058. Anal. Calcd for $\mathrm{C}_{12} \mathrm{H}_{15} \mathrm{FO}_{2}: \mathrm{C}, 68.55 ; \mathrm{H}, 7.19$. Found: C, 68.65; H, 7.16. 


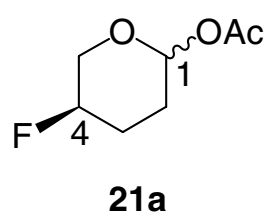

4-Fluorotetrahydropyran-1-acetate (21a): Palladium on carbon (10\%, $0.153 \mathrm{~g})$ was added to a solution of 4-fluoro-1-benzyloxytetrahydropyran $(0.168 \mathrm{~g}, 0.801 \mathrm{mmol})$ in $\mathrm{CD}_{2} \mathrm{Cl}_{2}(4 \mathrm{~mL})$ and the mixture was purged with $\mathrm{H}_{2}$, then allowed to stir in a sealed vessel at 1 atm. ${ }^{10}$ After filtering the solution, acetic anhydride $(0.378 \mathrm{~g}, 3.70 \mathrm{mmol})$, pyridine $(0.259 \mathrm{~mL}, 3.20 \mathrm{mmol})$, and 4-dimethylaminopyridine $(0.118 \mathrm{~g}, 0.962 \mathrm{mmol})$ were added. The reaction mixture was allowed to stir for $24 \mathrm{~h}$. Saturated aqueous $\mathrm{NH}_{4} \mathrm{Cl}(2 \mathrm{~mL})$ was added and the $\mathrm{CH}_{2} \mathrm{Cl}_{2}$ was removed in vacuo. The residue was dissolved in MTBE $(1.5 \mathrm{~mL})$. The organic layer was washed with saturated aqueous $\mathrm{Na}_{2} \mathrm{HPO}_{4}(4 \times 1 \mathrm{~mL}), \mathrm{NaH}_{2} \mathrm{PO}_{4}(4 \times 1 \mathrm{~mL})$, and $\mathrm{CuSO}_{4}(5 \times 1$ $\mathrm{mL}$ ), filtered through $\mathrm{Na}_{2} \mathrm{SO}_{4}$, and concentrated in vacuo. The resulting oil was purified by flash column chromatography (pentane to $1: 4 \mathrm{Et}_{2} \mathrm{O}$ /pentane) to afford the product (as a 1:1 mixture of isomers) as a colorless oil (0.085 g, 35\%): ${ }^{1} \mathrm{H} \mathrm{NMR}\left(500 \mathrm{MHz}, \mathrm{CDCl}_{3}\right) \delta 6.13$ (bs, $1 \mathrm{H}), 5.87(\mathrm{~m}, 1 \mathrm{H}), 4.73-4.55(\mathrm{~m}, 1 \mathrm{H}$ and dtt, $J=47.9,7.6,3.7,1 \mathrm{H}), 4.00-3.81(\mathrm{~m}, 4 \mathrm{H}), 2.20$ (m, 1H), $2.11(\mathrm{~m}, 3 \mathrm{H}), 2.10(\mathrm{~m}, 3 \mathrm{H}), 1.97(\mathrm{~m}, 5 \mathrm{H}), 1.81(\mathrm{~m}, 1 \mathrm{H}), 1.64(\mathrm{~m}, 1 \mathrm{H}) ;{ }^{13} \mathrm{C}$ NMR $(125$ $\left.\mathrm{MHz}, \mathrm{CDCl}_{3}\right) \delta 169.6,169.5,91.21,91.20,85.9(\mathrm{~d}, J=40), 84.6(\mathrm{~d}, J=40), 65.2(\mathrm{~d}, J=$ 26), 63.7 (d, $J=21), 26.3$ (d, $J=6), 25.7$ (d, $J=20), 23.2$ (d, $J=1), 22.4$ (d, $J=21), 21.1$ (d, $J$ = 3); ${ }^{19} \mathrm{~F} \mathrm{NMR} \mathrm{(376} \mathrm{MHz,} \mathrm{CDCl}_{3}$ ) $\delta-187.4$ (m), -190.1 (m); IR (thin film) 2960, 1751, 1441, $1373,1243,1211,1140,1050 \mathrm{~cm}^{-1}$; HRMS (EI/GCMS) $m / z$ calcd for $\mathrm{C}_{6} \mathrm{H}_{8} \mathrm{FO}_{3}\left(\mathrm{M}-\mathrm{CH}_{3}\right)^{+}$ 147.0457, found 147.0462. Anal. Calcd for $\mathrm{C}_{7} \mathrm{H}_{11} \mathrm{FO}_{3}: \mathrm{C}, 51.85 ; \mathrm{H}, 6.84$. Found: $\mathrm{C}, 52.12 ; \mathrm{H}$, 6.74 , 


\section{IIi. Synthesis of 4-Chlorotetrahydropyran-1-acetate (21b)}
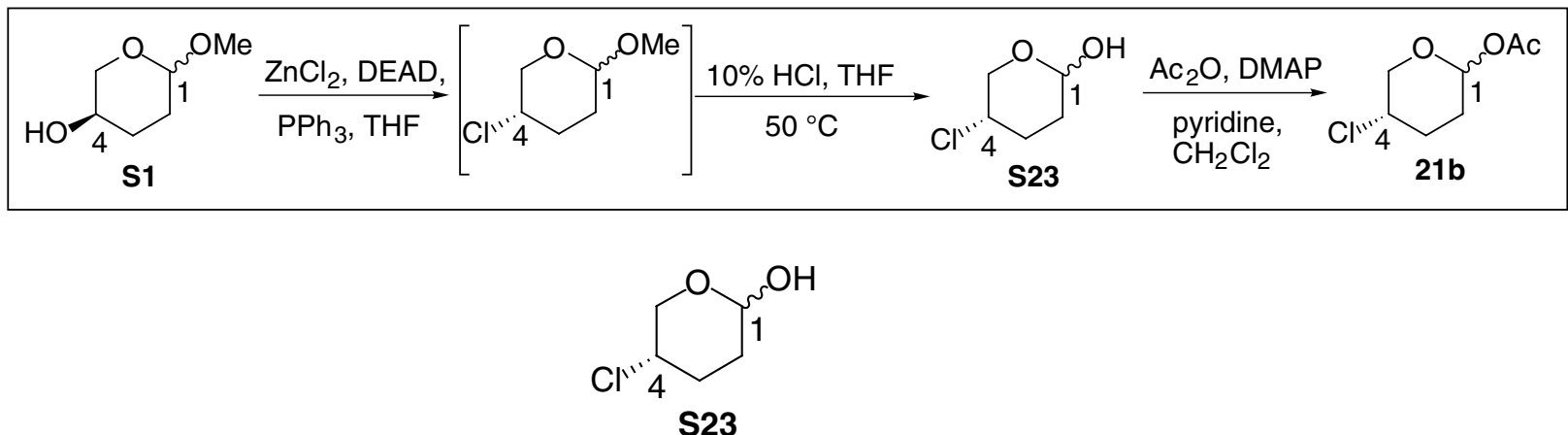

4-Chlorotetrahydropyran-1-ol (S23): ${ }^{11}$ A solution of anhydrous zinc chloride $(9.06 \mathrm{~g}, 66.5$ $\mathrm{mmol})$ in THF $(600 \mathrm{~mL})$ and a solution of diethyl azodicarboxylate $(34.8 \mathrm{~g}, 200 \mathrm{mmol})$ in THF $(120 \mathrm{~mL})$ were added consecutively to a solution of 4-hyroxy-1-methoxytetrahydropyran $(8.79$ $\mathrm{g}, 66.5 \mathrm{mmol})$ and triphenylphosphine $(52.3 \mathrm{~g}, 200 \mathrm{mmol})$ in THF $(600 \mathrm{~mL})$. The mixture was stirred at $23{ }^{\circ} \mathrm{C}$ for $10 \mathrm{~h}$ and concentrated in vacuo. The triphenylphosphine oxide was removed by flash chromatography (1:49 to $1: 9 \mathrm{Et}_{2} \mathrm{O}$ /pentane) to afford the impure product as a volatile, colorless oil. Because inseparable impurities were present, the impure methyl acetal was submitted to hydrolysis conditions. A $10 \%$ aqueous solution of $\mathrm{HCl}(200 \mathrm{~mL})$ was added slowly to a solution of methyl acetal in THF $(400 \mathrm{~mL})$. After $10 \mathrm{~h}$, the mixture was neutralized with saturated aqueous $\mathrm{NaHCO}_{3}$, extracted with $\mathrm{Et}_{2} \mathrm{O}(4 \times 150 \mathrm{~mL})$, dried over $\mathrm{MgSO}_{4}$, filtered, and concentrated in vacuo. The resulting oil was purified by flash chromatography (1:9 to 1:4 $\mathrm{Et}_{2} \mathrm{O} /$ pentane) to afford the product (as a 1:1 mixture of anomers) as a volatile colorless oil (4.26 g, 47\% over two steps): ${ }^{3}{ }^{1} \mathrm{H}$ NMR $\left(500 \mathrm{MHz} \mathrm{CDCl}_{3}\right) \delta 5.12(\mathrm{dd}, J=7.0,3.3,1 \mathrm{H}), 5.02(\mathrm{dt}, J$ $=7.6,2.8,1 \mathrm{H}), 4.21(\mathrm{ddd}, J=11.9,3.4,1.2,1 \mathrm{H}), 4.05(\mathrm{~m}, 1 \mathrm{H}), 3.99-3.95(\mathrm{~m}, 2 \mathrm{H}), 3.76(\mathrm{~m}$, $1 \mathrm{H}), 3.56(\mathrm{ddd}, J=11.9,6.7,0.9,1 \mathrm{H}), 2.81(\mathrm{~d}, J=3.3,1 \mathrm{H}), 2.67(\mathrm{~s}, 1 \mathrm{H}), 2.35(\mathrm{~m}, 1 \mathrm{H})$, 2.15-2.06 (m, 3H), $1.91(\mathrm{~m}, 1 \mathrm{H}), 1.86-1.78(\mathrm{~m}, 2 \mathrm{H}), 1.59(\mathrm{~m}, 1 \mathrm{H}) ;{ }^{13} \mathrm{C} \mathrm{NMR}(125 \mathrm{MHz}$ $\left.\mathrm{CDCl}_{3}\right) \delta 93.6,91.5,67.5,65.5,54.0,53.8,29.8,29.3,29.0,28.7$; IR (thin film) 3391, 2959, $1440 \mathrm{~cm}^{-1}$; HRMS (CI/isobutane) $m / z$ calcd for $\mathrm{C}_{5} \mathrm{H}_{9} \mathrm{O}_{2} \mathrm{Cl}(\mathrm{M})^{+} 136.0291$, found 136.0290. 


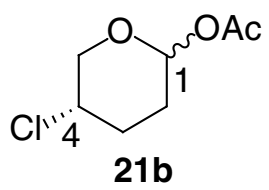

4-Chlorotetrahydropyran-1-acetate (21b): A solution of 4-chlorotetrahydropyran-1-ol (2.53 $\mathrm{g}, 18.5 \mathrm{mmol})$, acetic anhydride $(8.74 \mathrm{~mL}, 92.6 \mathrm{mmol})$, pyridine $(5.99 \mathrm{~mL}, 74.1 \mathrm{mmol})$ and $4-$ dimethylaminopyridine $(2.73 \mathrm{~g}, 22.2 \mathrm{mmol})$ in $\mathrm{CH}_{2} \mathrm{Cl}_{2}(250 \mathrm{~mL})$ was stirred for $24 \mathrm{~h}$. Saturated aqueous $\mathrm{NH}_{4} \mathrm{Cl}(50 \mathrm{~mL})$ was added, and $\mathrm{CH}_{2} \mathrm{Cl}_{2}$ was removed in vacuo. The residue was dissolved in MTBE (250 mL), and the layers were separated. The organic layer was washed with saturated aqueous $\mathrm{Na}_{2} \mathrm{HPO}_{4}(4 \times 50 \mathrm{~mL}), \mathrm{NaH}_{2} \mathrm{PO}_{4}(4 \times 50 \mathrm{~mL})$, and $\mathrm{CuSO}_{4}(5 \times 50 \mathrm{~mL})$, filtered through $\mathrm{Na}_{2} \mathrm{SO}_{4}$, and concentrated in vacuo. The resulting oil was purified by flash chromatography (pentane to $1: 4 \mathrm{Et}_{2} \mathrm{O}$ /pentane) to afford the product (as a 1:1 mixture of anomers) as a volatile, colorless oil $(3.28 \mathrm{~g}, 99 \%):{ }^{1}{ }^{1} \mathrm{H} \mathrm{NMR}\left(500 \mathrm{MHz}, \mathrm{CDCl}_{3}\right) \delta 6.03(\mathrm{t}, J=$ 2.7, $1 \mathrm{H}), 6.00(\mathrm{t}, J=3.5,1 \mathrm{H}), 4.18-4.14(\mathrm{~m}, 2 \mathrm{H}), 4.00(\mathrm{ddd}, J=15.0,10.6,4.5,1 \mathrm{H}), 3.87$ (ddd, $J=11.2,4.6,1.8,1 \mathrm{H}), 3.78(\mathrm{t}, J=10.8,1 \mathrm{H}), 3.72(\mathrm{~m}, 1 \mathrm{H}), 2.33(\mathrm{~m}, 1 \mathrm{H}), 2.28-2.17(\mathrm{~m}, 2 \mathrm{H})$, $2.13(\mathrm{~s}, 3 \mathrm{H}), 2.11(\mathrm{~s}, 3 \mathrm{H}), 2.07(\mathrm{~m}, 1 \mathrm{H}), 1.96-1.89(\mathrm{~m}, 3 \mathrm{H}), 1.67(\mathrm{~m}, 1 \mathrm{H}),{ }^{13} \mathrm{C} \mathrm{NMR}(125 \mathrm{MHz}$, $\left.\mathrm{CDCl}_{3}\right) \delta 169.5,169.3,91.7,90.0,67.3,66.1,53.9,52.7,28.8,28.7,27.6,25.1,21.0$; IR (thin film) 2965, 1738, $1371 \mathrm{~cm}^{-1}$; HRMS (CI/isobutane) $m / z$ calcd for $\mathrm{C}_{5} \mathrm{H}_{8} \mathrm{O}_{2} \mathrm{Cl}\left(\mathrm{M}-\mathrm{C}_{2} \mathrm{H}_{3} \mathrm{O}\right)^{+}$ 135.0213, found 135.0211

\section{IIJ. Synthesis of 4-Bromotetrahydropyran-1-acetate (21c)}

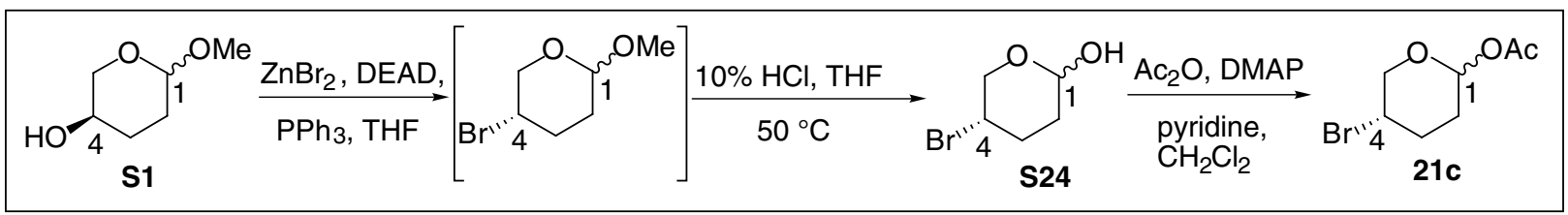




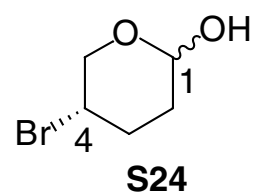

4-Bromotetrahydropyran-1-ol (S24): A solution of anhydrous zinc bromide (3.44 g, 15.3 $\mathrm{mmol})$ in THF $(150 \mathrm{~mL})$ and a solution of diethyl azodicarboxylate $(7.99 \mathrm{~g}, 45.9 \mathrm{mmol})$ in THF $(30 \mathrm{~mL})$ were added consecutively to a solution of 4-hydroxy-1-methoxytetrahydropyran (2.02 $\mathrm{g}, 15.3 \mathrm{mmol})$ and triphenylphosphine $(12.0 \mathrm{~g}, 45.9 \mathrm{mmol})$ in THF $(150 \mathrm{~mL})$. The mixture was stirred at $23{ }^{\circ} \mathrm{C}$ for $24 \mathrm{~h}$ and concentrated in vacuo. The triphenylphosphine oxide was removed by flash chromatography (1:9 $\mathrm{Et}_{2} \mathrm{O}$ /pentane) to afford the impure product as a colorless oil. Because inseparable impurities were present, the impure methyl acetal was submitted to hydrolysis conditions. An aqueous $(10 \%)$ solution of $\mathrm{HCl}(100 \mathrm{~mL})$ was added slowly to a solution of methyl acetal in THF $(200 \mathrm{~mL})$. After $36 \mathrm{~h}$, the mixture was neutralized with saturated aqueous $\mathrm{NaHCO}_{3}$, extracted with $\mathrm{Et}_{2} \mathrm{O}(4 \times 100 \mathrm{~mL})$, dried over $\mathrm{MgSO}_{4}$, filtered, and concentrated in vacuo. The product proved to be highly unstable, decomposing quickly upon concentration. The resulting oil could be quickly purified by flash chromatography (1:9 to 1:4 $\mathrm{Et}_{2} \mathrm{O} /$ pentane) to afford the product (as a 1:1 mixture of anomers) as a volatile, colorless oil $\left(2.67 \mathrm{~g}, 96 \%\right.$ over two steps): ${ }^{1} \mathrm{H}$ NMR $\left(500 \mathrm{MHz} \mathrm{CDCl}_{3}\right) \delta 5.19(\mathrm{dd}, J=6.6,3.2,1 \mathrm{H}), 5.01$ (m, 1H), $4.23(\mathrm{ddd}, J=12.0,3.6,1.5,1 \mathrm{H}), 4.20-4.07(\mathrm{~m}, 3 \mathrm{H}), 3.78(\mathrm{~m}, 1 \mathrm{H}), 3.64(\mathrm{dd}, J=12.0$, $7.2,1 \mathrm{H}), 2.80(\mathrm{~d}, J=4.8,1 \mathrm{H}), 2.63(\mathrm{dd}, J=3.8,1.7,1 \mathrm{H}), 2.39(\mathrm{~m}, 1 \mathrm{H}), 2.28(\mathrm{~m}, 1 \mathrm{H}), 2.22-2.07$ $(\mathrm{m}, 2 \mathrm{H}), 1.98-1.79(\mathrm{~m}, 3 \mathrm{H}), 1.61(\mathrm{~m}, 1 \mathrm{H}) ;{ }^{13} \mathrm{C} \mathrm{NMR}\left(125 \mathrm{MHz}, \mathrm{CDCl}_{3}\right) \delta 93.9,91.3,68.3$, 65.4, 46.1, 45.8, 31.0, 30.64, 30.61, 29.3; IR (thin film) 3396, 2957, 1451, 1057, $735 \mathrm{~cm}^{-1}$; HRMS (CI/isobutane) $m / z$ calcd for $\mathrm{C}_{5} \mathrm{H}_{9} \mathrm{O}_{2} \mathrm{Br}(\mathrm{M})^{+}$179.9786, found 179.9785. 


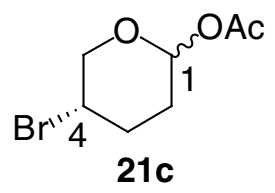

4-Bromotetrahydropyran-1-acetate (21c): A solution of 4-bromotertahydropyran-1-ol (1.88 g, $10.4 \mathrm{mmol})$, acetic anhydride $(4.90 \mathrm{~mL}, 51.9 \mathrm{mmol})$, pyridine $(3.36 \mathrm{~mL}, 41.5 \mathrm{mmol})$ and 4-dimethylaminopyridine $(1.53 \mathrm{~g}, 12.5 \mathrm{mmol})$ in $\mathrm{CH}_{2} \mathrm{Cl}_{2}(52 \mathrm{~mL})$ was stirred for $24 \mathrm{~h}$. Saturated aqueous $\mathrm{NH}_{4} \mathrm{Cl}(25 \mathrm{~mL})$ was added, and $\mathrm{CH}_{2} \mathrm{Cl}_{2}$ was removed in vacuo. The residue was dissolved in MTBE (150 mL), and the layers were separated. The organic layer was washed with saturated aqueous $\mathrm{Na}_{2} \mathrm{HPO}_{4}(4 \times 30 \mathrm{~mL}), \mathrm{NaH}_{2} \mathrm{PO}_{4}(4 \times 30 \mathrm{~mL})$, and $\mathrm{CuSO}_{4}(5 \times 30 \mathrm{~mL})$, filtered through $\mathrm{Na}_{2} \mathrm{SO}_{4}$, and concentrated in vacuo. The resulting oil was purified by flash chromatography (pentane to 1:4 $\mathrm{Et}_{2} \mathrm{O}$ /pentane) to afford the product (as a 1:1 mixture of anomers) as a volatile, colorless oil $(1.69 \mathrm{~g}, 73 \%):{ }^{1} \mathrm{H} \mathrm{NMR}\left(500 \mathrm{MHz}, \mathrm{CDCl}_{3}\right) \delta 6.09(\mathrm{t}, J=$ 2.7, $1 \mathrm{H}), 5.98(\mathrm{dd}, J=4.6,3.1,1 \mathrm{H}), 4.25(\mathrm{~m}, 1 \mathrm{H}), 4.19(\mathrm{dd}, J=12.5,3.0,1 \mathrm{H}), 4.10(\mathrm{~m}, 1 \mathrm{H})$, $3.91(\mathrm{~s}, 1 \mathrm{H}), 3.89(\mathrm{~s}, 1 \mathrm{H}), 3.78(\mathrm{ddd}, J=12.5,5.1,1.4,1 \mathrm{H}), 2.37(\mathrm{~m}, 1 \mathrm{H}), 2.30-2.17(\mathrm{~m}, 3 \mathrm{H})$, $2.13(\mathrm{~s}, 3 \mathrm{H}), 2.10(\mathrm{~s}, 3 \mathrm{H}), 2.01(\mathrm{~m}, 1 \mathrm{H}), 1.95-1.90(\mathrm{~m}, 2 \mathrm{H}), 1.69$ (ddt, $J=13.9,6.7,4.4,1 \mathrm{H})$; ${ }^{13} \mathrm{C} \mathrm{NMR}\left(125 \mathrm{MHz}, \mathrm{CDCl}_{3}\right) \delta 169.5,169.3,91.7,89.9,68.1,66.2,46.1,44.3,30.0,29.3,28.8$, 26.5, 21.01, 21.00; IR (thin film) 2939, 1749, 1372, 1220, 1009, $737 \mathrm{~cm}^{-1}$; HRMS (CI/isobutane) $m / z$ calcd for $\mathrm{C}_{5} \mathrm{H}_{8} \mathrm{BrO}_{2}\left(\mathrm{M}-\mathrm{C}_{2} \mathrm{H}_{3} \mathrm{O}\right)^{+}$178.9708, found 178.9704. Anal. Calcd for $\mathrm{C}_{7} \mathrm{H}_{11} \mathrm{BrO}_{3}$ : C, 37.69; H, 4.97. Found: C, 37.90; H, 5.05.

\section{IIK. Synthesis of 4-Iodotetrahydropyran-1-acetate (21d)}

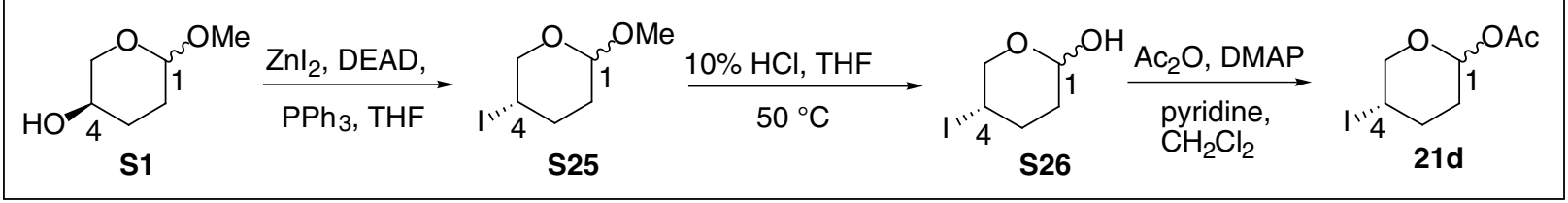




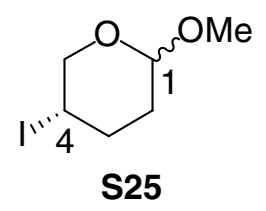

4-Iodo-1-methoxytetrahydropyran (S25): A solution of anhydrous zinc iodide $(2.41 \mathrm{~g}, 7.57$ $\mathrm{mmol})$ in THF (26 mL) and a solution of diethyl azodicarboxylate $(3.95 \mathrm{~g}, 22.7 \mathrm{mmol})$ in THF (5 mL) were added consecutively to a solution of 4-hydroxy-1-methoxytetrahydropyran $(1.00 \mathrm{~g}$, $7.57 \mathrm{mmol}$ ) and triphenylphosphine (5.95 g, $22.7 \mathrm{mmol})$ in THF (26 mL). The mixture was stirred at $25^{\circ} \mathrm{C}$ for $12 \mathrm{~h}$ and concentrated in vacuo. The resulting oil was purified by flash column chromatography (pentane to $1: 9 \mathrm{Et}_{2} \mathrm{O}$ /pentane) to afford one isomer as a colorless oil (0.751g, 41\%): ${ }^{1} \mathrm{H}$ NMR (500 MHz, $\left.\mathrm{CDCl}_{3}\right) \delta 4.76(\mathrm{~m}, 1 \mathrm{H}), 4.22(\mathrm{~m}, 1 \mathrm{H}), 3.97(\mathrm{t}, J=11.1$, 1H), $3.78(\mathrm{~m}, 1 \mathrm{H}), 3.36(\mathrm{~s}, 3 \mathrm{H}), 2.38(\mathrm{~m}, 1 \mathrm{H}), 2.21(\mathrm{~m}, 1 \mathrm{H}), 1.87(\mathrm{~m}, 1 \mathrm{H}), 1.73(\mathrm{~m}, 1 \mathrm{H}) ;{ }^{13} \mathrm{C}$ NMR (125 MHz, $\left.\mathrm{CDCl}_{3}\right) \delta 97.0,66.0,54.8,32.9,31.3,24.2 ;$ IR (thin film) 2929, 1123, 1050, $1019 \mathrm{~cm}^{-1}$; HRMS (EI/GCMS) $m / z$ calcd for $\mathrm{C}_{6} \mathrm{H}_{11} \mathrm{IO}_{2}(\mathrm{M})^{+} 241.9804$, found 241.9803 . Anal. Calcd for $\mathrm{C}_{6} \mathrm{H}_{11} \mathrm{O}_{2}$ : C, 29.77; H, 4.58. Found: C, 29.85; H, 4.55.

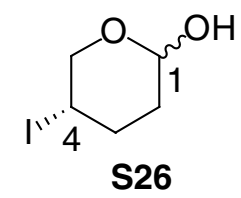

4-Iodotetrahydropyran-1-ol (S26): A 10\% aqueous solution of $\mathrm{HCl}(41 \mathrm{~mL})$ was added slowly to a solution of 4-iodo-1-methoxytetrahydropyran $(1.83 \mathrm{~g}, 0.008 \mathrm{mmol})$ in THF (81 $\mathrm{mL}$ ). The reaction mixture was stirred at $55{ }^{\circ} \mathrm{C}$ for $12 \mathrm{hr}$ and allowed to cool to $23{ }^{\circ} \mathrm{C}$. The mixture was neutralized with saturated aqueous $\mathrm{NaHCO}_{3}$ and extracted with $\mathrm{Et}_{2} \mathrm{O}(3 \times 30 \mathrm{~mL})$. The organic layers were dried over $\mathrm{MgSO}_{4}$, filtered, and concentrated in vacuo. The resulting oil was purified by flash column chromatography $\left(1: 9 \mathrm{Et}_{2} \mathrm{O} /\right.$ pentane to $3: 2 \mathrm{Et}_{2} \mathrm{O}$ /pentane $)$ to afford 
the product (as a 1:1 mixture of anomers) as a colorless oil ( $0.76 \mathrm{~g}, 45 \%):{ }^{1} \mathrm{H}$ NMR (400 MHz, $\left.\mathrm{CDCl}_{3}\right) \delta 5.29(\mathrm{~d}, J=3.1,1 \mathrm{H}), 4.95(\mathrm{~m}, 1 \mathrm{H}) 4.24-4.15(\mathrm{~m}, 4 \mathrm{H}), 3.77-3.68(\mathrm{~m}, 2 \mathrm{H}), 3.02(\mathrm{~m}$, 1H) $2.78(\mathrm{~m}, 1 \mathrm{H}), 2.43-2.35(\mathrm{~m}, 2 \mathrm{H}), 2.20-2.16(\mathrm{~m}, 1 \mathrm{H}), 2.07-2.00(\mathrm{~m}, 2 \mathrm{H}), 1.89-1.80(\mathrm{~m}$, 2H), 1.65-1.60 (m, 1H); ${ }^{13} \mathrm{C}$ NMR (125 MHz, $\left.\mathrm{CDCl}_{3}\right) \delta$ 94.4, 91.1, 71.0, 66.8, 32.4, 32.6, 30.9, 24.4, 24.3; IR (thin film) 3391, 2952, 1449, 1270, 1133, $1050 \mathrm{~cm}^{-1}$; HRMS (EI/GCMS) $\mathrm{m} / z$ calcd for $\mathrm{C}_{5} \mathrm{H}_{9} \mathrm{IO}_{2}(\mathrm{M})^{+} 227.9649$, found 227.9641 .

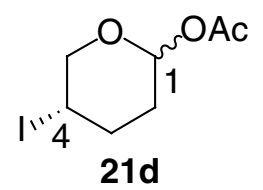

4-Iodotetrahydropyran-1-acetate (21d): A solution of 4-iodotetrahydropyran-1-ol (0.779 g, $3.42 \mathrm{mmol})$, acetic anhydride $(1.61 \mathrm{~mL}, 17.1 \mathrm{mmol})$, pyridine $(1.10 \mathrm{~mL}, 13.7 \mathrm{mmol})$, and $4-$ dimethylaminopyridine $(0.500 \mathrm{~g}, 4.09 \mathrm{mmol})$ in $\mathrm{CH}_{2} \mathrm{Cl}_{2}(34 \mathrm{~mL})$ was stirred for $12 \mathrm{~h}$. Saturated aqueous $\mathrm{NH}_{4} \mathrm{Cl}(10 \mathrm{~mL})$ was added and the $\mathrm{CH}_{2} \mathrm{Cl}_{2}$ layer was separated and removed in vacuo. The residue was dissolved in MTBE (34 mL) and washed with aqueous $\mathrm{Na}_{2} \mathrm{HPO}_{4}(3$ $\times 10 \mathrm{~mL}), \mathrm{NaH}_{2} \mathrm{PO}_{4}(3 \times 10 \mathrm{~mL})$, and $\mathrm{CuSO}_{4}(5 \times 10 \mathrm{~mL})$. The organic phases were filtered through $\mathrm{Na}_{2} \mathrm{SO}_{4}$ and concentrated in vacuo. The resulting oil was purified by flash chromatography (pentane to $1: 10 \mathrm{Et}_{2} \mathrm{O} /$ pentane) to afford the product (as a 2:3 mixture of isomers) as a colorless oil $(0.776 \mathrm{~g}, 84 \%):{ }^{1} \mathrm{H}$ NMR $\left(500 \mathrm{MHz}, \mathrm{CDCl}_{3}\right) \delta 6.18(\mathrm{t}, J=2.0,1 \mathrm{H})$, $5.90(\mathrm{~m}, 1 \mathrm{H}) 4.33-4.30(\mathrm{~m}, 1 \mathrm{H}), 4.26-4.20(\mathrm{~m}, 1 \mathrm{H}), 4.10-4.06(\mathrm{dd}, J=12.4,3.3,1 \mathrm{H}) 4.02(\mathrm{t}, J$ $=11.3,1 \mathrm{H}), 3.90-3.87(\mathrm{ddd}, J=11.3,4.5,1.8,1 \mathrm{H}), 3.81-3.70(\mathrm{dd}, J=12.4,6.4,1 \mathrm{H})$, 2.35-2.25 (m, 3H), $2.13(\mathrm{~s}, 3 \mathrm{H}$ and m, 1H), $2.09(\mathrm{~s}, 3 \mathrm{H}), 2.07(\mathrm{~m}, 1 \mathrm{H}), 2.05(\mathrm{~m}, 1 \mathrm{H}), 1.83(\mathrm{~m}$, 1H), $1.75(\mathrm{~m}, 1 \mathrm{H}) ;{ }^{13} \mathrm{C} \mathrm{NMR}\left(125 \mathrm{MHz}, \mathrm{CDCl}_{3}\right) \delta 170.0,169.7,92.5,90.5,71.0,68.3,32.1$, 32.0, 31.5, 29.7, 24.7, 22.7, 21.6, 21.5; IR (thin film) 2959, 1749, 1371, 1242, 1129, $1049 \mathrm{~cm}^{-1}$; HRMS (EI/GCMS) $m / z$ calcd for $\mathrm{C}_{5} \mathrm{H}_{8} \mathrm{IO}_{1}\left(\mathrm{M}-\mathrm{C}_{2} \mathrm{H}_{3} \mathrm{O}_{2}\right)^{+} 210.9620$, found 210.9622 . 


\section{IIL. Synthesis of 2-Isopropyltetrahydropyran-1-acetate (32c)}
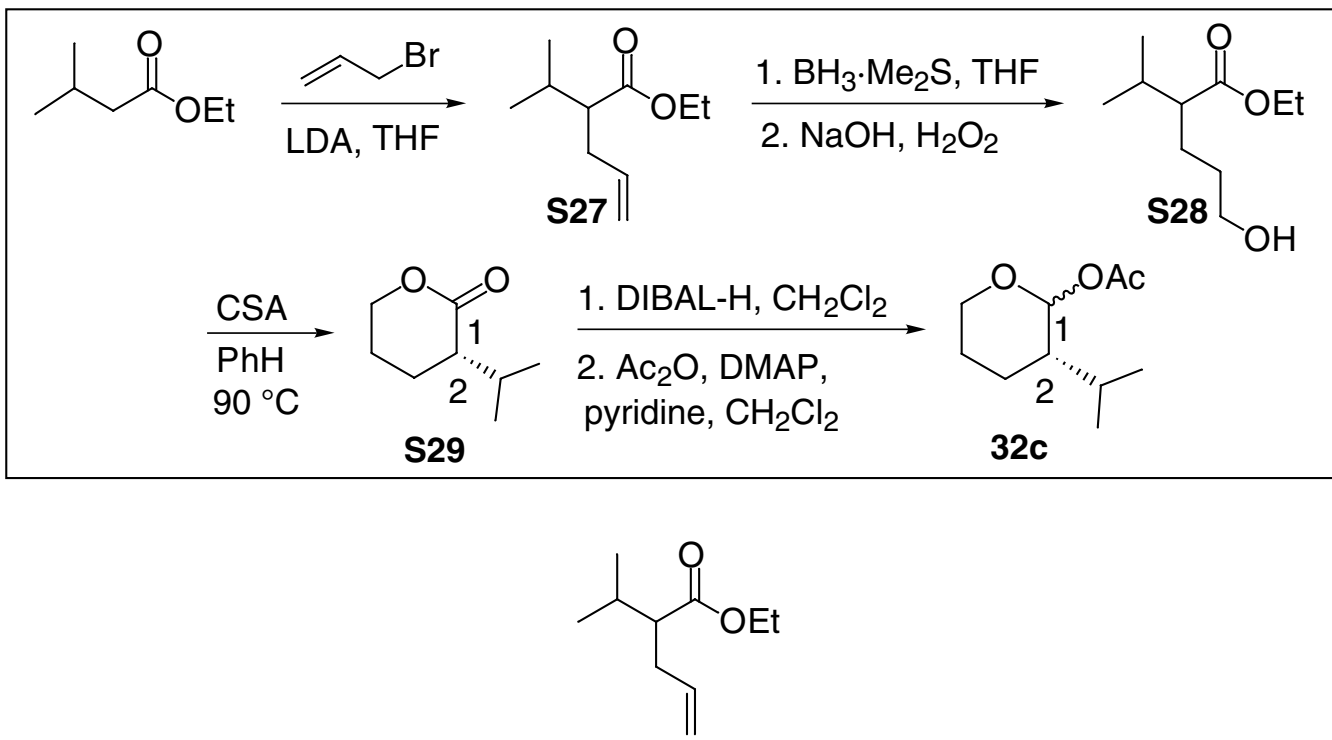

S27

Ethyl 2-isopropyl-4-pentenoate (S27): ${ }^{12}$ To a stirred solution of diisopropylamine (11.9 $\mathrm{mL}, 72.2 \mathrm{mmol})$ in THF $(91 \mathrm{~mL})$ at $-78{ }^{\circ} \mathrm{C}$ was added a solution of $n$-BuLi in hexanes $(34 \mathrm{~mL}$, $2.5 \mathrm{M})$. The resultant pale solution was allowed to stir at $-78{ }^{\circ} \mathrm{C}$ for $1 \mathrm{~h}$. A solution of isovalerate $(10.10 \mathrm{~g}, 77.6 \mathrm{mmol})$ in THF $(24 \mathrm{~mL})$ was added, and the mixture was allowed to stir for $4 \mathrm{~h}$ at $-78{ }^{\circ} \mathrm{C}$. Allyl bromide $(31 \mathrm{~mL}, 82 \mathrm{mmol})$ was added, and the mixture was allowed to warm to $23{ }^{\circ} \mathrm{C}$ and stirred for $16 \mathrm{~h}$ before addition of $2 \mathrm{~N} \mathrm{HCl}(100 \mathrm{~mL})$. The mixture was poured into $\mathrm{Et}_{2} \mathrm{O}(200 \mathrm{~mL})$ and the layers were separated. The aqueous layer was extracted with $\mathrm{Et}_{2} \mathrm{O}(3 \times 200 \mathrm{~mL})$ and the combined organic layers were washed with saturated aqueous $\mathrm{NaHCO}_{3}(65 \mathrm{~mL})$, dried over $\mathrm{MgSO}_{4}$, filtered, and concentrated in vacuo. The resulting oil was purified by distillation $\left(100{ }^{\circ} \mathrm{C}, 760 \mathrm{mmHg}\right)$ to afford the product as a colorless oil $(7.78 \mathrm{~g}$, 63\%): ${ }^{1} \mathrm{H}$ NMR (500 MHz, $\left.\mathrm{CDCl}_{3}\right) \delta 5.78-5.70(\mathrm{~m}, 1 \mathrm{H}), 5.07-5.03$ (ddd, $J=17.1,3.3,1.4$, $1 \mathrm{H}), 4.98(\mathrm{~m}, 1 \mathrm{H}), 4.15-4.10(\mathrm{~m}, 2 \mathrm{H}), 2.36-2.25(\mathrm{~m}, 2 \mathrm{H}), 2.22-2.17$ (ddd, $J=13.1,7.3,4.4$, $1 \mathrm{H}), 1.92-1.85(\mathrm{~m}, 1 \mathrm{H}), 1.25(\mathrm{t}, J=7.1,3 \mathrm{H}), 0.96(\mathrm{~d}, J=6.8,3 \mathrm{H}), 0.93(\mathrm{~d}, J=6.8,3 \mathrm{H}) ;{ }^{13} \mathrm{C}$ NMR (125 MHz, $\left.\mathrm{CDCl}_{3}\right) \delta 175.1,136.2,116.4,60.1,52.6,34.1,30.4,20.5,20.4,14.5$; IR 
(thin film) 3079, 2964, 1735, 1642, 1373, 1180, $1039 \mathrm{~cm}^{-1}$; HRMS (EI/GCMS) $\mathrm{m} / \mathrm{z}$ calcd for $\mathrm{C}_{10} \mathrm{H}_{18} \mathrm{O}_{2}(\mathrm{M})^{+}$170.1307, found 170.1304 .

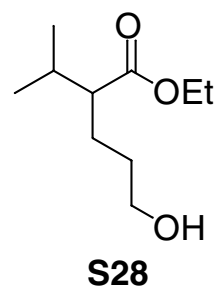

Ethyl 2-isopropyl-5-ol-pentanoate (S28): To a solution of ethyl 2-isopropyl-4-pentenoate $(8.88 \mathrm{~g}, 52.2 \mathrm{mmol})$ in $\mathrm{THF}(43 \mathrm{~mL})$ at $0{ }^{\circ} \mathrm{C}$ was added $10 \mathrm{M} \mathrm{BH}_{3} \cdot \mathrm{SMe}_{2}(2.1 \mathrm{~mL})$. The reaction mixture was allowed to warm to $23{ }^{\circ} \mathrm{C}$ and stirred for $72 \mathrm{~h}$. The reaction mixture was cooled to $0{ }^{\circ} \mathrm{C}$ and $10 \% \mathrm{NaOH}(24 \mathrm{~mL})$ followed by $30 \% \mathrm{H}_{2} \mathrm{O}_{2}(12 \mathrm{~mL})$ were added. The reaction mixture was allowed to warm to $23{ }^{\circ} \mathrm{C}$ and stirred for $48 \mathrm{~h}$ before $\mathrm{K}_{2} \mathrm{CO}_{3}(25 \mathrm{~g})$ was added and the mixture was stirred for an additional $2 \mathrm{~h}$. The layers were separated, and the aqueous layer was extracted with $\mathrm{Et}_{2} \mathrm{O}(3 \times 50 \mathrm{~mL})$. The combined organic phases were dried over $\mathrm{MgSO}_{4}$, filtered, and concentrated in vacuo. The resulting yellow oil was purified by flash column chromatography $\left(1: 9 \mathrm{Et}_{2} \mathrm{O}\right.$ /pentane to $2: 3 \mathrm{Et}_{2} \mathrm{O} /$ pentane $)$ to afford the product as a colorless oil (4.81 g, 49\%): ${ }^{1} \mathrm{H}$ NMR (400 $\left.\mathrm{MHz} \mathrm{CDCl}_{3}\right) \delta 4.21-4.15(\mathrm{~m}, 2 \mathrm{H}), 3.66(\mathrm{~m}, 2 \mathrm{H})$, 2.18-2.13 (ddd, $J=9.9,7.3,4.4,1 \mathrm{H}), 1.92-1.87(\mathrm{~m}, 1 \mathrm{H}), 1.68-1.57(\mathrm{~m}, 5 \mathrm{H}), 1.30$ (t, $J=7.1$, $3 \mathrm{H}), 0.97(\mathrm{~d}, J=6.9,3 \mathrm{H}), 0.95(\mathrm{~d}, J=6.8,3 \mathrm{H}) ;{ }^{13} \mathrm{C}$ NMR $\left(125 \mathrm{MHz}, \mathrm{CDCl}_{3}\right)$ $\delta 176.1,62.8,60.2,52.7,31.2,31.0,25.9,20.7,20.4,14.6$; IR (thin film) 3431, 2961, 1731, 1467, 1376, 1194, $1029 \mathrm{~cm}^{-1}$; HRMS (EI/GCMS) $m / z$ calcd for $\mathrm{C}_{10} \mathrm{H}_{21} \mathrm{O}_{3}(\mathrm{M}+\mathrm{H})^{+} 189.1491$, found 189.1478 . 


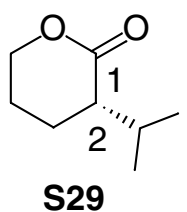

2-Isopropyltetrahydropyran-1-one (S29): To a solution of ethyl 2-isopropyl-5-olpentenoate $(0.691 \mathrm{~g}, 3.67 \mathrm{mmol})$ in benzene $(122 \mathrm{~mL})$ was added camphorsulfonic acid (0.069 g, $0.30 \mathrm{mmol}$ ). The mixture was stirred at $80{ }^{\circ} \mathrm{C}$ for $24 \mathrm{~h}$, cooled to $23{ }^{\circ} \mathrm{C}$ and concentrated in vacuo. The resulting oil was purified by flash column chromatography (pentane to 1:4 $\mathrm{Et}_{2} \mathrm{O} /$ pentane) to afford the product as a light yellow oil $(0.370 \mathrm{~g}, 71 \%):{ }^{1} \mathrm{H} \mathrm{NMR}(500 \mathrm{MHz}$, $\left.\mathrm{CDCl}_{3}\right) \delta 4.33(\mathrm{~m}, 1 \mathrm{H}), 4.23(\mathrm{ddd}, J=11.1,7.9,4.6,1 \mathrm{H}), 2.40(\mathrm{~m}, 2 \mathrm{H}), 1.92(\mathrm{~m}, 3 \mathrm{H}), 1.62(\mathrm{~m}$, $1 \mathrm{H}), 1.00(\mathrm{~d}, J=6.8,3 \mathrm{H}), 0.94(\mathrm{~d}, J=6.7,3 \mathrm{H}) ;{ }^{13} \mathrm{C} \mathrm{NMR}\left(125 \mathrm{MHz}, \mathrm{CDCl}_{3}\right)$ $\delta 173.8,68.8,46.1,28.8,22.6,20.3,20.1,18.3$; IR (thin film) 2962, 1731, 1465, 1389, 1273, $1155,1085 \mathrm{~cm}^{-1}$; HRMS (EI/GCMS) $m / z$ calcd for $\mathrm{C}_{8} \mathrm{H}_{14} \mathrm{O}_{2}(\mathrm{M})^{+}$142.0994, found 142.0993. Anal. Calcd for $\mathrm{C}_{8} \mathrm{H}_{14} \mathrm{O}_{2}:$ C, 67.57; H, 9.92. Found: C, 67.27; H, 10.03.

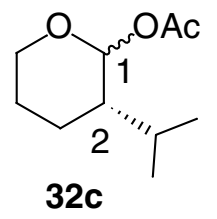

2-Isopropylterahydropyran-1-acetate (32c): A solution of 2-isopropyl-tetrahydropyran-1one $(0.367 \mathrm{~g}, 2.57 \mathrm{mmol})$ in $\mathrm{CH}_{2} \mathrm{Cl}_{2}(26 \mathrm{~mL})$ was cooled to $-78{ }^{\circ} \mathrm{C}$ and a $1.5 \mathrm{M}$ solution of DIBAL-H in toluene $(2.06 \mathrm{~mL}, 3.09 \mathrm{mmol})$ was added dropwise. After $5 \mathrm{~h}$, acetic anhydride (1.21 mL, $12.9 \mathrm{mmol})$, pyridine $(0.83 \mathrm{~mL}, 10.3 \mathrm{mmol})$, and 4-dimethylaminopyridine $(0.474 \mathrm{~g}$, $3.86 \mathrm{mmol}$ ) were added. The reaction mixture was allowed to warm slowly over $0.5 \mathrm{~h}$ to $23{ }^{\circ} \mathrm{C}$ and it was stirred for $24 \mathrm{~h}$. Saturated aqueous $\mathrm{NH}_{4} \mathrm{Cl}(18 \mathrm{~mL})$ was added, and the $\mathrm{CH}_{2} \mathrm{Cl}_{2}$ was removed in vacuo. The residue was dissolved in MTBE $(28 \mathrm{~mL})$. The organic layer was washed with saturated aqueous $\mathrm{Na}_{2} \mathrm{HPO}_{4}(4 \times 12 \mathrm{~mL}), \mathrm{NaH}_{2} \mathrm{PO}_{4}(4 \times 12 \mathrm{~mL})$, and $\mathrm{CuSO}_{4}(5 \times 12 \mathrm{~mL})$, filtered through $\mathrm{Na}_{2} \mathrm{SO}_{4}$, and concentrated in vacuo. The resulting oil was purified by flash 
column chromatography (pentane to $1: 4 \mathrm{Et}_{2} \mathrm{O}$ /pentane) to afford the product (as a 1:2 mixture of isomers) as a colorless oil: (0.196 g, 41\%): ${ }^{1} \mathrm{H}$ NMR $\left(500 \mathrm{MHz}, \mathrm{CDCl}_{3}\right) \delta 6.13(\mathrm{~d}, J=2.1$, $1 \mathrm{H}), 5.70(\mathrm{~d}, J=6.4,1 \mathrm{H}), 3.94(\mathrm{~m}, 1 \mathrm{H}), 3.72(\mathrm{td}, J=11.2,3.1,1 \mathrm{H}), 3.67(\mathrm{~m}, 1 \mathrm{H}), 3.57(\mathrm{ddd}, J=$ 11.4, 8.2, 3.5, 1H), 2.11, (s, 6H), $1.86(\mathrm{~m}, 4 \mathrm{H}), 1.67(\mathrm{~m}, 2 \mathrm{H}), 1.53-1.37(\mathrm{~m}, 6 \mathrm{H}), 0.95(\mathrm{~d}, J=6.9$, $3 \mathrm{H}), 0.89(\mathrm{~d}, J=6.4,3 \mathrm{H}), 0.88(\mathrm{~d}, J=6.3,3 \mathrm{H}), 0.87(\mathrm{~d}, J=6.9,3 \mathrm{H}) ;{ }^{13} \mathrm{C} \mathrm{NMR}(125 \mathrm{MHz}$, $\left.\mathrm{CDCl}_{3}\right) \delta 170.3,170.0,95.4,92.6,65.2,61.2,45.4,44.4,29.2,26.7,25.5,23.8,22.2,21.43$, 21.36, 21.2, 20.9, 20.5, 20.4, 18.6; IR (thin film) 2960, 2876, 1751, 1371, $1232 \mathrm{~cm}^{-1}$; HRMS (EI/GCMS) $m / z$ calcd for $\mathrm{C}_{8} \mathrm{H}_{15} \mathrm{O}\left(\mathrm{M}-\mathrm{C}_{2} \mathrm{H}_{3} \mathrm{O}_{2}\right)^{+}$126.1045, found 126.1046. Anal. Calcd for $\mathrm{C}_{10} \mathrm{H}_{18} \mathrm{O}_{3}$ : C, 64.49; H, 9.74. Found: C, 64.40; H, 9.84.

\section{IIM. Synthesis of 2-tert-Butyltetrahydropyran-1-acetate (32d)}

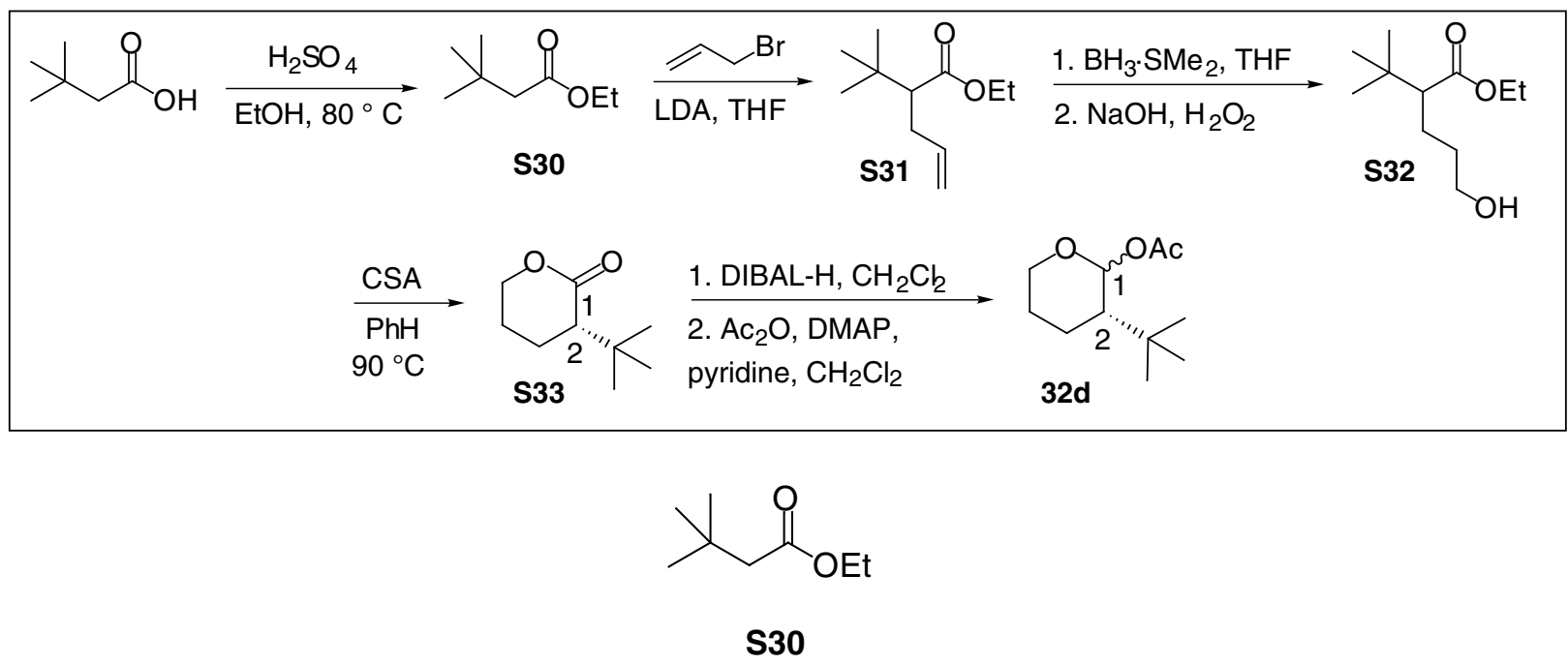

Ethyl 3,3-dimethyl-butanoate (S30): To a solution of ethyl 2-tert-butylacetic acid (13.0 g, $112 \mathrm{mmol})$ in $\mathrm{EtOH}(75 \mathrm{~mL})$ was added $36 \mathrm{~N} \mathrm{H}_{2} \mathrm{SO}_{4}(3.4 \mathrm{~mL})$. The reaction mixture was heated to $84{ }^{\circ} \mathrm{C}$ for $24 \mathrm{~h}$. The reaction mixture was cooled to $23{ }^{\circ} \mathrm{C}$, and the acid was quenched with saturated aqueous $\mathrm{NaHCO}_{3}$ and the layers were separated. The aqueous layer was extracted with $\mathrm{Et}_{2} \mathrm{O}(3 \times 100 \mathrm{~mL})$. The combined organic layers were washed with $\mathrm{H}_{2} \mathrm{O}$, dried 
over $\mathrm{MgSO}_{4}$, filtered, and concentrated in vасио. The resulting oil was purified by distillation $\left(83{ }^{\circ} \mathrm{C}, 760 \mathrm{mmHg}\right)$ to afford the product as a colorless oil $(12.0 \mathrm{~g}, 74 \%):{ }^{1} \mathrm{H}$ NMR $(500 \mathrm{MHz}$, $\left.\mathrm{CDCl}_{3}\right) \delta 4.12(\mathrm{~m}, 2 \mathrm{H}), 2.18(\mathrm{~d}, J=1.8,2 \mathrm{H}), 1.25(\mathrm{~m}, 3 \mathrm{H}), 1.03(\mathrm{~s}, 9 \mathrm{H}) ;{ }^{13} \mathrm{C} \mathrm{NMR}(125 \mathrm{MHz}$ $\left.\mathrm{CDCl}_{3}\right) \delta 172.7,60.2,48.4,31.1,30.0,14.7$; IR (thin film) 2960, 1737, 1469, 1368, 1231, 1132, $1047 \mathrm{~cm}^{-1}$; HRMS (EI/GCMS) $m / z$ calcd for $\mathrm{C}_{8} \mathrm{H}_{16} \mathrm{O}_{2}(\mathrm{M})^{+}$144.1150, found 144.1145. Anal. Calcd for $\mathrm{C}_{8} \mathrm{H}_{16} \mathrm{O}_{2}$ : C, 66.63; $\mathrm{H}, 11.18$. Found: C, 66.38; H, 11.25.

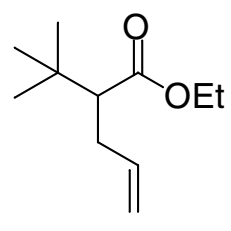

S31

Ethyl 2-tert-butyl-4-pentenoate (S31): To a stirred solution of diisopropylamine (13.4 mL, $88.7 \mathrm{mmol})$ in THF $(94 \mathrm{~mL})$ at $-78^{\circ} \mathrm{C}$ was added a solution of $n$-BuLi in hexanes $(35 \mathrm{~mL}, 2.5$ M). The resultant pale solution was allowed to stir at $-78{ }^{\circ} \mathrm{C}$ for $1 \mathrm{~h}$. A solution of ethyl 3,3dimethyl-butanoate (11.62 $\mathrm{g}, 80.6 \mathrm{mmol})$ in THF $(25 \mathrm{~mL})$ was added, and the mixture was allowed to stir for $4 \mathrm{~h}$ at $-78{ }^{\circ} \mathrm{C}$. Allyl bromide (32 $\mathrm{mL}, 85 \mathrm{mmol}$ ) was added, and the mixture was allowed to warm to $23^{\circ} \mathrm{C}$ and stirred for $16 \mathrm{~h}$ before addition of $2 \mathrm{~N} \mathrm{HCl}(100 \mathrm{~mL})$. The mixture was poured into $\mathrm{Et}_{2} \mathrm{O}(200 \mathrm{~mL})$ and the layers were separated. The aqueous layer was extracted with $\mathrm{Et}_{2} \mathrm{O}(3 \times 200 \mathrm{~mL})$ and the combined organic layers were washed with saturated aqueous $\mathrm{NaHCO}_{3}(65 \mathrm{~mL})$, dried over $\mathrm{MgSO}_{4}$, and filtered. The resulting oil was purified by distillation $\left(115{ }^{\circ} \mathrm{C}, 760 \mathrm{mmHg}\right)$ to afford the product as a colorless oil $(11.75 \mathrm{~g}, 89 \%):{ }^{1} \mathrm{H}$ $\operatorname{NMR}\left(500 \mathrm{MHz}, \mathrm{CDCl}_{3}\right) \delta 5.71(\mathrm{~m}, 1 \mathrm{H}), 5.05(\mathrm{~m}, 1 \mathrm{H}), 4.96(\mathrm{~m}, 1 \mathrm{H}), 4.11(\mathrm{q}, J=7.2,2 \mathrm{H})$, $2.36(\mathrm{~m}, 1 \mathrm{H}), 2.22(\mathrm{~m}, 2 \mathrm{H}), 1.24(\mathrm{t}, J=7.2,3 \mathrm{H}), 0.98(\mathrm{~s}, 9 \mathrm{H}) ;{ }^{13} \mathrm{C}$ NMR $\left(125 \mathrm{MHz}, \mathrm{CDCl}_{3}\right)$ $\delta 174.7,136.6,116.3,59.9,56.2,33.0,32.3,28.0,14.6$; IR (thin film) $\mathrm{cm}^{-1} 2964,1732,1642$, 1475, 1371, 1152; HRMS (EI/GCMS) $m / z$ calcd for $\mathrm{C}_{11} \mathrm{H}_{21} \mathrm{O}_{2}(\mathrm{M}+\mathrm{H})^{+}$184.1542, found 185.1543. 


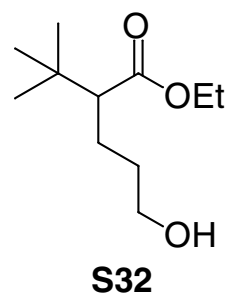

Ethyl 2-tert-butyl-5-ol-pentanoate (S32): To a solution of ethyl 2-tert-butyl-4-pentenoate (5.02 g, $27.2 \mathrm{mmol})$ in THF $(23 \mathrm{~mL})$ at $0{ }^{\circ} \mathrm{C}$ was added $10 \mathrm{M} \mathrm{BH}_{3} \cdot \mathrm{SMe}_{2}(1.1 \mathrm{~mL})$. The reaction mixture was allowed to warm to $23{ }^{\circ} \mathrm{C}$ for $72 \mathrm{~h}$. The reaction mixture was cooled to 0 ${ }^{\circ} \mathrm{C}$, and $10 \% \mathrm{NaOH}(13 \mathrm{~mL})$ followed by $30 \% \mathrm{H}_{2} \mathrm{O}_{2}(6.2 \mathrm{~mL})$ were added. The reaction mixture was allowed to warm to $23{ }^{\circ} \mathrm{C}$ and stirred for $48 \mathrm{~h}$ before $\mathrm{K}_{2} \mathrm{CO}_{3}(14 \mathrm{~g})$ was added and the mixture was stirred for an additional $2 \mathrm{~h}$. The layers were separated, and the aqueous layer was extracted with $\mathrm{Et}_{2} \mathrm{O}(3 \times 40 \mathrm{~mL})$. The combined organic phases were dried over $\mathrm{MgSO}_{4}$, filtered, and concentrated in vacuo. The resulting yellow oil was purified by flash column chromatography $\left(1: 9 \mathrm{Et}_{2} \mathrm{O} /\right.$ pentane to $2: 3 \mathrm{Et}_{2} \mathrm{O} /$ pentane) to afford the product as a colorless oil $(2.81 \mathrm{~g}, 51 \%):{ }^{1} \mathrm{H}$ NMR $\left(500 \mathrm{MHz}, \mathrm{CDCl}_{3}\right) \delta 4.14(\mathrm{q}, J=7.1,2 \mathrm{H}), 3.63(\mathrm{~m}, 2 \mathrm{H}), 2.14(\mathrm{dd}, J=$ 12.0, 2.9, 1H), $1.73(\mathrm{~m}, 1 \mathrm{H}), 1.61(\mathrm{~m}, 1 \mathrm{H}), 1.45(\mathrm{~m}, 2 \mathrm{H}), 1.27(\mathrm{t}, J=7.1,3 \mathrm{H}), 0.96(\mathrm{~s}, 9 \mathrm{H}) ;{ }^{13} \mathrm{C}$ NMR (125 MHz, $\left.\mathrm{CDCl}_{3}\right) \delta 175.7,62.8,60.0,56.3,33.2,31.8,28.0,24.1,14.6$; IR (thin film) 3440, 2960, 1729, 1371, 1151, $1054 \mathrm{~cm}^{-1}$; HRMS (EI/GCMS) $m / z$ calcd for $\mathrm{C}_{11} \mathrm{H}_{22} \mathrm{O}_{3}(\mathrm{M})^{+}$ 202.1569, found 202.1563. Anal. Calcd for $\mathrm{C}_{11} \mathrm{H}_{22} \mathrm{O}_{3}$ : C, 65.31; H, 10.96. Found: C, 65.24; $\mathrm{H}$, 10.90 .

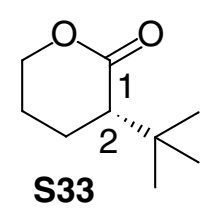

2-tert-Butyltetrahydropyran-1-one (S33): To a solution of ethyl 2-tert-butyl-5-olpentenoate $(1.22 \mathrm{~g}, 6.03 \mathrm{mmol})$ in benzene $(201 \mathrm{~mL})$ was added camphorsulfonic acid $(0.560 \mathrm{~g}$, 
$2.41 \mathrm{mmol}$ ). The mixture was stirred at $80{ }^{\circ} \mathrm{C}$ for $24 \mathrm{~h}$, then it was cooled to $23{ }^{\circ} \mathrm{C}$ and concentrated in vacuo. The resulting oil was purified by flash column chromatography (pentane to $1: 4 \mathrm{Et}_{2} \mathrm{O} /$ pentane) to afford the product as a colorless oil $(0.763 \mathrm{~g}, 81 \%):{ }^{1} \mathrm{H}$ NMR (500 $\left.\mathrm{MHz}, \mathrm{CDCl}_{3}\right) \delta 4.27(\mathrm{~m}, 1 \mathrm{H}), 4.23(\mathrm{dt}, J=10.9,5.9,1 \mathrm{H}), 2.32(\mathrm{dd}, J=11.5,8.2,1 \mathrm{H}), 2.09(\mathrm{dq}$ $J=13.5,7.1,1 \mathrm{H}), 1.87(\mathrm{~m}, 2 \mathrm{H}), 1.65(\mathrm{~m}, 1 \mathrm{H}), 1.08(\mathrm{~s}, 9 \mathrm{H}) ;{ }^{13} \mathrm{C} \mathrm{NMR}\left(125 \mathrm{MHz}, \mathrm{CDCl}_{3}\right)$ $\delta 173.3,68.1,49.3,33.3,28.0,22.9,21.5$; IR (thin film) 2962, 1732, 1483, 1380, 1252, 1129, $1082 \mathrm{~cm}^{-1}$; HRMS (EI/GCMS) $m / z$ calcd for $\mathrm{C}_{5} \mathrm{H}_{8} \mathrm{O}_{2}\left(\mathrm{M}-\mathrm{C}_{4} \mathrm{H}_{8}\right)^{+}$100.0524, found 100.0527. Anal. Calcd for $\mathrm{C}_{9} \mathrm{H}_{16} \mathrm{O}_{2}$ : C, 69.19; H, 10.32. Found: C, 68.76; H, 10.16.

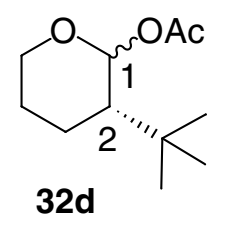

2-tert-Butylterahydropyran-1-acetate (32d): A solution of 2-tert-butyltetrahydropyran-1one, $(0.759 \mathrm{~g}, 4.86 \mathrm{mmol})$ in $\mathrm{CH}_{2} \mathrm{Cl}_{2}(49 \mathrm{~mL})$ was cooled to $-78{ }^{\circ} \mathrm{C}$, and a $1.5 \mathrm{M}$ solution of DIBAL-H in toluene $(3.9 \mathrm{~mL}, 5.8 \mathrm{mmol})$ was added dropwise. After $5 \mathrm{~h}$, acetic anhydride $(2.29$ $\mathrm{mL}, 24.3 \mathrm{mmol})$, pyridine $(1.57 \mathrm{~mL}, 19.4 \mathrm{mmol})$, and 4-dimethylaminopyridine (0.715 g, 5.83 mmol) were added. The reaction mixture was allowed to warm slowly over $0.5 \mathrm{~h}$ to $23{ }^{\circ} \mathrm{C}$ and it was stirred for $24 \mathrm{~h}$. Saturated aqueous $\mathrm{NH}_{4} \mathrm{Cl}(25 \mathrm{~mL})$ was added, and the $\mathrm{CH}_{2} \mathrm{Cl}_{2}$ was removed in vacuo. The residue was dissolved in MTBE $(34 \mathrm{~mL})$. The organic layer was washed with saturated aqueous $\mathrm{Na}_{2} \mathrm{HPO}_{4}(4 \times 15 \mathrm{~mL}), \mathrm{NaH}_{2} \mathrm{PO}_{4}(4 \times 15 \mathrm{~mL})$, and $\mathrm{CuSO}_{4}(5 \times$ $15 \mathrm{~mL}$ ), filtered through $\mathrm{Na}_{2} \mathrm{SO}_{4}$, and concentrated in vacuo. The resulting oil was purified by flash column chromatography (pentane to $1: 4 \mathrm{Et}_{2} \mathrm{O}$ /pentane) to afford the product as a colorless oil $(0.341 \mathrm{~g}, 35 \%):{ }^{1} \mathrm{H}$ NMR $\left(500 \mathrm{MHz}, \mathrm{CDCl}_{3}\right) \delta 6.16(\mathrm{~d}, J=2.5,1 \mathrm{H}), 3.75(\mathrm{td}, J=11.4,3.2$, 1H), $3.67(\mathrm{~m}, 1 \mathrm{H}), 2.12(\mathrm{~s}, 3 \mathrm{H}), 1.73-1.67(\mathrm{~m}, 4 \mathrm{H}), 1.57(\mathrm{~m}, 1 \mathrm{H}), 0.89(\mathrm{~s}, 9 \mathrm{H}) ;{ }^{13} \mathrm{C}$ NMR $(125$ $\left.\mathrm{MHz}, \mathrm{CDCl}_{3}\right) \delta 170.2,92.9,61.6,48.7,32.1,28.4,26.4,21.8,19.8 ; \mathrm{IR}$ (thin film) 2957, 2874, 1751, 1474, 1084, $1011 \mathrm{~cm}^{-1}$; HRMS (EI/GCMS) $m / z$ calcd for $\mathrm{C}_{10} \mathrm{H}_{17} \mathrm{O}_{3}\left(\mathrm{M}-\mathrm{CH}_{3}\right)^{+}$ 
185.1178, found 185.1174. Anal. Calcd for $\mathrm{C}_{11} \mathrm{H}_{20} \mathrm{O}_{3}$ : C, 65.97; H, 10.07. Found: C, 66.27; H, 9.98 .

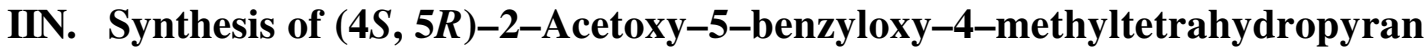

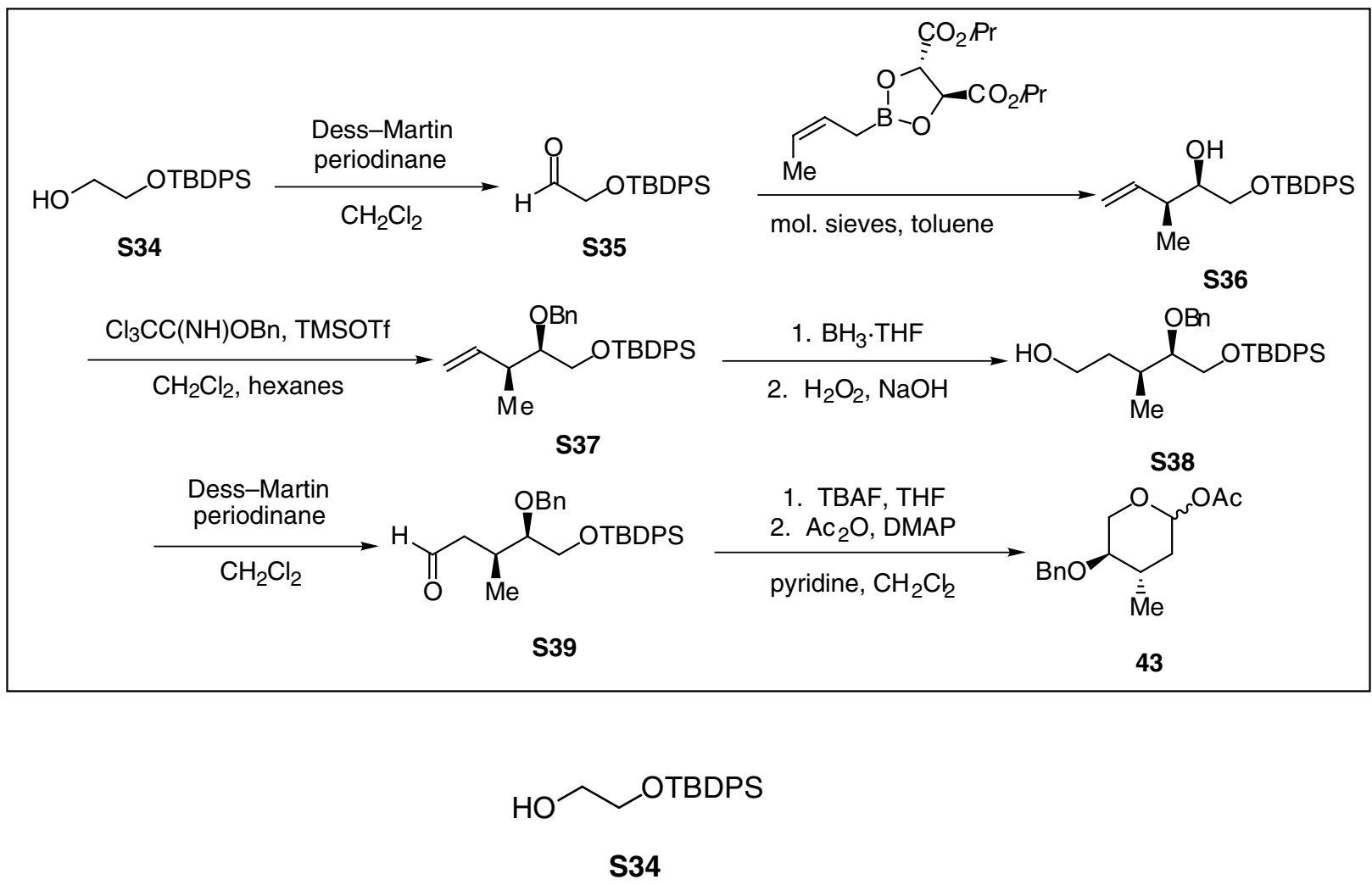

2-(tert-Butyldiphenylsilyloxy)ethanol (S34): Sodium hydride (0.873 g, $36.4 \mathrm{mmol})$ was suspended in THF $(70 \mathrm{~mL})$ in a flame-dried flask after being washed with hexanes $(3 \times 5 \mathrm{~mL})$. Ethylene glycol $(2.03 \mathrm{~mL}, 36.4 \mathrm{mmol})$ was added at $22{ }^{\circ} \mathrm{C}$, and the mixture was stirred for 45 min. tert-Butylchlorodiphenylsilane $(9.5 \mathrm{~mL}, 37 \mathrm{mmol})$ was then added. After five days, the mixture was poured into diethyl ether $(500 \mathrm{~mL})$, washed with $10 \%$ aqueous $\mathrm{K}_{2} \mathrm{CO}_{3}(170 \mathrm{~mL})$ and brine $(170 \mathrm{~mL})$, dried over $\mathrm{MgSO}_{4}$, filtered and concentrated in vacuo. The resulting oil was purified by flash chromatography (1:4 EtOAc/hexanes) to afford the product as a colorless oil $(6.83 \mathrm{~g}, 62 \%):{ }^{1} \mathrm{H}$ NMR $\left(500 \mathrm{MHz}, \mathrm{CDCl}_{3}\right) \delta 7.67(\mathrm{dd}, J=7.9,1.4,4 \mathrm{H}), 7.45-7.37(\mathrm{~m}$, $6 \mathrm{H}), 3.73(\mathrm{~m}, 2 \mathrm{H}), 3.67(\mathrm{~m}, 2 \mathrm{H}), 2.12(\mathrm{t}, J=6.3,1 \mathrm{H}), 1.07(\mathrm{~s}, 9 \mathrm{H}) ;{ }^{13} \mathrm{C} \mathrm{NMR}(125 \mathrm{MHz}$ 
$\left.\mathrm{CDCl}_{3}\right) \delta 135.5,133.3,129.8,127.8,65.0,63.7,26.8,19.2$; IR (thin film) 3411, 3071, 2932, 2858, 1056, $823 \mathrm{~cm}^{-1}$; HRMS (CI/isobutane) $m / z$ calcd for $\mathrm{C}_{14} \mathrm{H}_{15} \mathrm{O}_{2} \mathrm{Si}\left(\mathrm{M}-t\right.$-Butyl) ${ }^{+}$ 243.0841, found 243.0840. Anal. Calcd for $\mathrm{C}_{18} \mathrm{H}_{24} \mathrm{O}_{2} \mathrm{Si}: \mathrm{C}, 71.95 ; \mathrm{H}, 8.05$. Found: C, 71.93; H, 8.10.

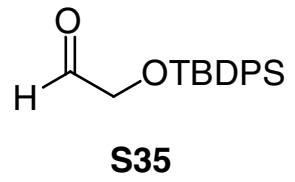

(tert-Butyldiphenylsilyloxy)acetaldehyde (S35): Dess-Martin periodinane ${ }^{6,7}$ (11.08 g, $26.0 \mathrm{mmol})$ was added to a solution of 2-(tert-butyldiphenylsilyloxy)ethanol $(6.00 \mathrm{~g}, 20.0$ mmol) in $\mathrm{CH}_{2} \mathrm{Cl}_{2}(200 \mathrm{~mL})$. The mixture was stirred for $12 \mathrm{~h}$ before adding $100 \mathrm{~mL}$ of a saturated aqueous solution of $\mathrm{Na}_{2} \mathrm{~S}_{2} \mathrm{O}_{3}$ and $\mathrm{NaHCO}_{3}$. Stirring was continued for $40 \mathrm{~min}$ before the layers were separated. The organic layer was washed with saturated aqueous $\mathrm{NaHCO}_{3}(100$ $\mathrm{mL})$ and water $(100 \mathrm{~mL})$ and dried over $\mathrm{Na}_{2} \mathrm{SO}_{4}$. Solvent was removed in vacuo, and the resulting oil was purified by flash chromatography (1:9 EtOAc/hexanes) to afford the known product as a colorless oil $(4.63 \mathrm{~g}, 78 \%):{ }^{13}{ }^{1} \mathrm{HNMR}\left(500 \mathrm{MHz}, \mathrm{CDCl}_{3}\right) \delta 9.73(\mathrm{~d}, J=0.8,1 \mathrm{H})$, $7.66(\mathrm{~m}, 4 \mathrm{H}), 7.45-7.38(\mathrm{~m}, 6 \mathrm{H}), 4.21(\mathrm{~d}, J=0.8,2 \mathrm{H}), 1.10(\mathrm{~s}, 9 \mathrm{H}) ;{ }^{13} \mathrm{C}$ NMR $(125 \mathrm{MHz}$ $\left.\mathrm{CDCl}_{3}\right) \delta 201.7,135.5,132.5,130.1,127.9,70.0,26.7,19.2$; IR (thin film) 3071, 2958, 2932, 2858, 1738, $1114 \mathrm{~cm}^{-1}$; HRMS (CI/isobutane) $m / z$ calcd for $\mathrm{C}_{14} \mathrm{H}_{13} \mathrm{O}_{2} \mathrm{Si}\left(\mathrm{M}-t\right.$-Butyl) ${ }^{+}$ 241.0685 , found 241.0686 . 


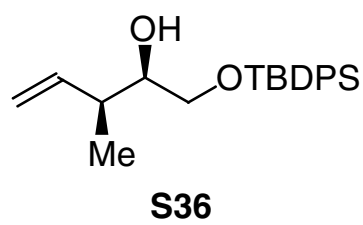

(2R,3S)-1-(tert-Butyldiphenylsilyloxy)-3-methylpent-4-en-2-ol $\quad(\mathbf{S 3 6}): \quad(Z)-(2 S, 3 S)-$ Diisopropyltartrate crotylboronate was added as a $0.44 \mathrm{M}$ solution in toluene $(85.0 \mathrm{~mL}, 37$ $\mathrm{mmol})^{14}$ to a suspension of crushed $4 \AA$ molecular sieves $(0.789 \mathrm{~g})$ in toluene $(50 \mathrm{~mL})$. The mixture was cooled to $-78{ }^{\circ} \mathrm{C}$, and a solution of (tert-butyldiphenylsilyloxy)acetaldehyde (4.00 $\mathrm{g}, 13.4 \mathrm{mmol})^{13}$ in toluene $(10 \mathrm{~mL})$ was added dropwise over $15 \mathrm{~min}$. After $4 \mathrm{~h}, 10 \%$ aqueous $\mathrm{NaOH}(50 \mathrm{~mL})$ was added. The mixture was warmed to $0{ }^{\circ} \mathrm{C}$, stirred for $45 \mathrm{~min}$, and filtered through Celite. The layers were separated, and the aqueous layer was extracted with $\mathrm{Et}_{2} \mathrm{O}(4 \times$ $30 \mathrm{~mL}$ ). The combined organic layers were dried over $\mathrm{K}_{2} \mathrm{CO}_{3}$ and concentrated in vacuo. The resulting oil was purified by flash chromatography (1:9 EtOAc/hexanes) to afford the product as a colorless oil (3.70 g, 78\%, >95:5 diastereoselectivity, as determined based on GC analysis): $[\alpha]_{\mathrm{D}}^{22}+1.7^{\circ}\left(c 0.72, \mathrm{CHCl}_{3}\right) ;{ }^{1} \mathrm{H}$ NMR $\left(500 \mathrm{MHz}, \mathrm{CDCl}_{3}\right) \delta 7.67-7.65(\mathrm{~m}, 4 \mathrm{H}), 7.46-7.37(\mathrm{~m}$, $6 \mathrm{H}), 5.64(\mathrm{ddd}, J=17.2,10.3,7.9,1 \mathrm{H}), 4.99(\mathrm{ddd}, J=17.2,1.7,1.2,1 \mathrm{H}), 4.94(\mathrm{ddd}, J=10.3$, $1.8,0.8,1 \mathrm{H}), 3.70(\mathrm{dd}, J=10.0,3.3,1 \mathrm{H}), 3.57(\mathrm{dd}, J=10.0,7.2,1 \mathrm{H}), 3.53(\mathrm{dt}, J=7.0,3.5,1 \mathrm{H})$, $2.52(\mathrm{~d}, J=3.9,1 \mathrm{H}), 2.31(\mathrm{~m}, 1 \mathrm{H}), 1.06(\mathrm{~s}, 9 \mathrm{H}), 1.05(\mathrm{~d}, J=6.8,3 \mathrm{H}) ;{ }^{13} \mathrm{C} \mathrm{NMR}(125 \mathrm{MHz}$ $\left.\mathrm{CDCl}_{3}\right) \delta 140.3,135.5,133.1,129.8,127.8,115.0,74.9,66.1,40.8,26.8,19.2,15.8$; IR (thin film) 3575, 3478, 3071, 2930, 1428, 1112, 917, 740, $702 \mathrm{~cm}^{-1}$; HRMS (CI/isobutane) $\mathrm{m} / \mathrm{z}$ calcd for $\mathrm{C}_{18} \mathrm{H}_{21} \mathrm{O}_{2} \mathrm{Si}\left(\mathrm{M}-t\right.$-Butyl) ${ }^{+}$297.1311, found 297.1303. Anal. Calcd for $\mathrm{C}_{22} \mathrm{H}_{30} \mathrm{O}_{2} \mathrm{Si}$ : C, 74.53; H, 8.53. Found: C, 74.68; H, 8.66. 


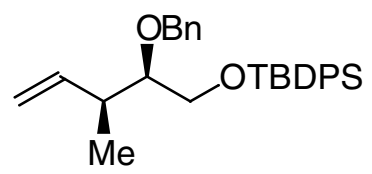

S37

(2R,3S)-(2-Benzyloxy-3-methylpent-4-enyloxy)-tert-butyldiphenylsilane (S37): Freshly distilled benzyl trichloroacetimidate $(0.311 \mathrm{~mL}, 1.67 \mathrm{mmol})$ was added to a solution of $(2 R, 3 S)$ 1-(tert-butyldiphenylsilyloxy)-3-methylpent-4-en-2-ol (0.100 g, $0.28 \mathrm{mmol})$ in $\mathrm{CH}_{2} \mathrm{Cl}_{2}(2 \mathrm{~mL})$ and hexanes $(3 \mathrm{~mL})$, and the mixture was cooled to $0{ }^{\circ} \mathrm{C}$. TMSOTf $(0.011 \mathrm{~mL}, 0.06 \mathrm{mmol})$ was added dropwise, and the mixture was warmed to $22{ }^{\circ} \mathrm{C}$ and stirred for $12 \mathrm{~h}$. Additional benzyl trichloroacetimidate $(0.150 \mathrm{~mL}, 0.81 \mathrm{mmol})$ and TMSOTf $(0.007 \mathrm{~mL}, 0.04 \mathrm{mmol})$ were added at $0{ }^{\circ} \mathrm{C}$, and the mixture was warmed to $22{ }^{\circ} \mathrm{C}$ and stirred for $48 \mathrm{~h}$. The solvent was removed in vacuo, and the residue was dissolved in $6: 1$ petroleum ether/ $\mathrm{Et}_{2} \mathrm{O}(20 \mathrm{~mL})$ and filtered through a plug of silica gel. The silica gel was rinsed with $\mathrm{Et}_{2} \mathrm{O}(2 \times 2 \mathrm{~mL})$, and the combined organic phases were washed with saturated aqueous $\mathrm{NaHCO}_{3}(10 \mathrm{~mL})$ and water $(10 \mathrm{~mL})$, dried over $\mathrm{Na}_{2} \mathrm{SO}_{4}$, and concentrated in vacuo. The resulting oil was purified by flash chromatography $\left(2: 1\right.$ hexanes/benzene) to afford the product as a colorless oil $(0.103 \mathrm{~g}, 82 \%):[\alpha]_{\mathrm{D}}^{22}-15.1^{\circ}(c$ 0.36, $\left.\mathrm{CHCl}_{3}\right) ;{ }^{1} \mathrm{H} \mathrm{NMR}\left(500 \mathrm{MHz}, \mathrm{CDCl}_{3}\right) \delta 7.69-7.67(\mathrm{~m}, 4 \mathrm{H}), 7.43-7.25(\mathrm{~m}, 11 \mathrm{H}), 5.78$ $(\mathrm{ddd}, J=17.3,10.3,7.6,1 \mathrm{H}), 5.01(\mathrm{dt}, J=17.2,1.5,1 \mathrm{H}), 4.95(\mathrm{~d}, J=10.3,1 \mathrm{H}), 4.71(\mathrm{~d}, J=$ 11.6, 1H), $4.52(\mathrm{~d}, J=11.6,1 \mathrm{H}), 3.74(\mathrm{~m}, 2 \mathrm{H}), 3.37(\mathrm{dd}, J=10.4,5.7,1 \mathrm{H}), 2.52(\mathrm{~m}, 1 \mathrm{H}), 1.06$ (s, 9H), $1.02(\mathrm{~d}, J=6.9,3 \mathrm{H}) ;{ }^{13} \mathrm{C} \mathrm{NMR}\left(125 \mathrm{MHz}, \mathrm{CDCl}_{3}\right) \delta 141.3,139.0,135.7,135.6,133.6$, $133.5,129.6,128.2,127.7,127.6,127.3,114.3,83.6,72.9,64.6,39.5,26.8,19.2,15.3$; IR (thin film) 3071, 2930, 1428, 1112, 823, 739, $701 \mathrm{~cm}^{-1}$; HRMS (CI/isobutane) $m / z$ calcd for $\mathrm{C}_{29} \mathrm{H}_{35} \mathrm{O}_{2} \mathrm{Si}(\mathrm{M}-\mathrm{H})^{+}$443.2406, found 443.2414. Anal. Calcd for $\mathrm{C}_{29} \mathrm{H}_{36} \mathrm{O}_{2} \mathrm{Si}: \mathrm{C}, 78.33$; $\mathrm{H}$, 8.16. Found: C, 78.61; H, 8.10. 


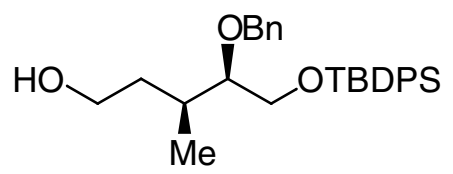

S38

(2R,3S)-2-Benzyloxy-1-(tert-butyldiphenylsilyloxy)-3-methylpentan-5-ol (S38): A 1 M solution of $\mathrm{BH}_{3}$. THF in THF (2.27 mL) was added to a solution of (2R,3S)-(2-benzyloxy-3methylpent-4-enyloxy)-tert-butyldiphenylsilane (1.01 g, $2.27 \mathrm{mmol})$ in THF $(20 \mathrm{~mL})$. The mixture was stirred for $15 \mathrm{~h}$ before dropwise addition of water $(1 \mathrm{~mL})$, addition of $10 \%$ aqueous $\mathrm{NaOH}(1.5 \mathrm{~mL})$, and dropwise addition of $30 \%$ aqueous $\mathrm{H}_{2} \mathrm{O}_{2}(1 \mathrm{~mL})$. The mixture was stirred for $5 \mathrm{~h}$ before the solvent was removed in vacuo. The residue was redissolved in $\mathrm{Et}_{2} \mathrm{O}$ (100 $\mathrm{mL})$, washed with water $(50 \mathrm{~mL})$ and brine $(50 \mathrm{~mL})$, dried over $\mathrm{MgSO}_{4}$, and concentrated in vасио. The resulting oil was purified by flash chromatography (1:4 EtOAc/hexanes) to afford the product as a colorless oil $(0.722 \mathrm{~g}, 69 \%):[\alpha]_{\mathrm{D}}{ }^{22}-20.7^{\circ}\left(c 0.09, \mathrm{CHCl}_{3}\right) ;{ }^{1} \mathrm{H} \quad \mathrm{NMR}(500$ $\left.\mathrm{MHz}, \mathrm{CDCl}_{3}\right) \delta 7.68(\mathrm{~m}, 4 \mathrm{H}), 7.44-7.25(\mathrm{~m}, 11 \mathrm{H}), 4.73(\mathrm{~d}, J=11.7,1 \mathrm{H}), 4.53(\mathrm{~d}, J=11.7$, $1 \mathrm{H}), 3.85(\mathrm{dd}, J=10.9,6.4,1 \mathrm{H}), 3.73(\mathrm{dd}, J=10.9,4.5,1 \mathrm{H}), 3.63(\mathrm{~m}, 1 \mathrm{H}), 3.56(\mathrm{~m}, 1 \mathrm{H}), 3.46$ $(\mathrm{m}, 1 \mathrm{H}), 1.96(\mathrm{~m}, 1 \mathrm{H}), 1.80(\mathrm{t}, J=5.4,1 \mathrm{H}), 1.63(\mathrm{~m}, 1 \mathrm{H}), 1.43(\mathrm{~m}, 1 \mathrm{H}), 1.06(\mathrm{~s}, 9 \mathrm{H}), 0.86(\mathrm{~d}, J$ $=7.0,3 \mathrm{H}) ;{ }^{13} \mathrm{C} \mathrm{NMR}\left(125 \mathrm{MHz}, \mathrm{CDCl}_{3}\right) \delta 138.8,135.6,133.5,129.7,128.3,127.7,127.5$, 83.5, 72.8, 64.4, 61.3, 36.3, 32.3, 26.9, 19.2, 15.5; IR (thin film) 3399, 3070, 2931, 1428, 1112, 738, $701 \mathrm{~cm}^{-1}$; HRMS (CI/isobutane) $m / z$ calcd for $\mathrm{C}_{29} \mathrm{H}_{39} \mathrm{O}_{3} \mathrm{Si}(\mathrm{M}+\mathrm{H})^{+} 463.2668$, found 463.2656. Anal. Calcd for $\mathrm{C}_{29} \mathrm{H}_{38} \mathrm{O}_{3} \mathrm{Si}$ : C, 75.28; H, 8.28. Found: C, 74.99; H, 8.25.

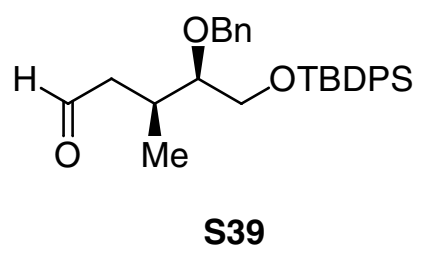

(3S,4R)-4-Benzyloxy-5-(tert-butyldiphenylsilyloxy)-3-methylpentanal

(S39):

Dess-Martin periodinane ${ }^{5,6}(0.794 \mathrm{~g}, 1.87 \mathrm{mmol})$ was added to a solution of $(2 R, 3 S)-2$ benzyloxy-1-(tert-butyldiphenylsilyloxy)-3-methylpentan-5-ol (0.722 g, $1.56 \mathrm{mmol})$ in $\mathrm{CH}_{2} \mathrm{Cl}_{2}$ 
$(50 \mathrm{~mL})$. The mixture was stirred for $14 \mathrm{~h}$. A saturated aqueous solution of $\mathrm{NaHCO}_{3}$ and $\mathrm{Na}_{2} \mathrm{~S}_{2} \mathrm{O}_{3}(30 \mathrm{~mL})$ was added. After stirring for $15 \mathrm{~min}$, the layers were separated, and the aqueous layer was extracted with $\mathrm{CH}_{2} \mathrm{Cl}_{2}(3 \times 15 \mathrm{~mL})$. The combined organic phases were washed with saturated aqueous $\mathrm{NaHCO}_{3}(30 \mathrm{~mL})$, dried over $\mathrm{Na}_{2} \mathrm{SO}_{4}$, and concentrated in vасио. The resulting oil was purified by flash chromatography (benzene) to afford the product as a colorless oil $(0.606 \mathrm{~g}, 84 \%):[\alpha]_{\mathrm{D}}{ }^{22}-32.5^{\circ}\left(c 0.04, \mathrm{CHCl}_{3}\right) ;{ }^{1} \mathrm{H} \mathrm{NMR}\left(500 \mathrm{MHz}, \mathrm{CDCl}_{3}\right) \delta$ $9.69(\mathrm{dd}, J=2.3,1.8,1 \mathrm{H}), 7.68(\mathrm{~m}, 4 \mathrm{H}), 7.46-7.25(\mathrm{~m}, 11 \mathrm{H}), 4.62(\mathrm{~d}, J=11.8,1 \mathrm{H}), 4.42(\mathrm{~d}, J=$ $11.8,1 \mathrm{H}), 3.78(\mathrm{dd}, J=10.9,5.7,1 \mathrm{H}), 3.70(\mathrm{dd}, J=10.9,5.2,1 \mathrm{H}), 3.43(\mathrm{~m}, 1 \mathrm{H}), 2.54(\mathrm{ddd}, J=$ 16.3, 5.8, 1.7, 1H), $2.46(\mathrm{~m}, 1 \mathrm{H}), 2.27(\mathrm{ddd}, J=16.2,7.4,2.5,1 \mathrm{H}), 1.06(\mathrm{~s}, 9 \mathrm{H}), 0.93(\mathrm{~d}, J=6.9$, $3 \mathrm{H}) ;{ }^{13} \mathrm{C} \mathrm{NMR}\left(125 \mathrm{MHz}, \mathrm{CDCl}_{3}\right) \delta 202.4,138.7,135.6,133.4,133.3,129.7,128.3,127.74$, 127.71, 127.5, 82.0, 72.5, 63.9, 47.7, 30.0, 26.8, 19.2, 14.8; IR (thin film) 3070, 2931, 1725, 1428, 1112, 824, 740, $702 \mathrm{~cm}^{-1}$; HRMS (CI/isobutane) $m / z$ calcd for $\mathrm{C}_{25} \mathrm{H}_{27} \mathrm{O}_{3} \mathrm{Si}(\mathrm{M}-t$ Butyl) ${ }^{+}$403.1729, found 403.1730. Anal. Calcd. for $\mathrm{C}_{29} \mathrm{H}_{36} \mathrm{O}_{3} \mathrm{Si}$ : C, 75.61; H, 7.88. Found: C, $74.53 ; \mathrm{H}, 7.77$.

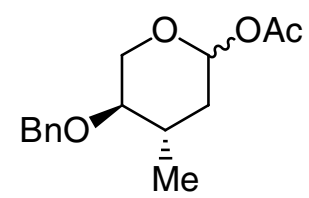

43

(4S,5R)-2-Acetoxy-5-benzyloxy-4-methyltetrahydropyran (43): Tetrabutylammonium fluoride was added as a $1 \mathrm{M}$ solution in THF $(26.3 \mathrm{~mL})$ to $(3 S, 4 R)-4$-Benzyloxy-5-(tertbutyldiphenylsilyloxy)-3-methylpentanal $(0.606 \mathrm{~g}, 1.32 \mathrm{mmol})$ and stirred for $48 \mathrm{~h}$. The solvent was removed in vacuo, and the majority of the tetrabutylammonium fluoride was removed by flash chromatography (4:1 hexanes/EtOAc). The resulting hemiacetal was isolated as a 45:55 $(\alpha: \beta)$ mixture of anomers with approximately $28 \mathrm{~mol} \%$ of tetrabutylammonium fluoride, as determined based on analysis of ${ }^{1} \mathrm{H}$ NMR spectroscopy. The unpurified yellow oil was 
dissolved in pyridine $(32 \mathrm{~mL})$ and acetic anhydride $(16 \mathrm{~mL}, 170 \mathrm{mmol})$. 4Dimethylaminopyridine $(0.020 \mathrm{~g}, 0.16 \mathrm{mmol})$ was added, and the mixture was stirred for $48 \mathrm{~h}$. The mixture was poured into a separatory funnel with tert-butyl methyl ether $(100 \mathrm{~mL})$, washed with saturated aqueous $\mathrm{NaHSO}_{3}(30 \mathrm{~mL})$, saturated aqueous $\mathrm{Na}_{2} \mathrm{HPO}_{4}(3 \times 30 \mathrm{~mL})$, saturated aqueous $\mathrm{NaH}_{2} \mathrm{PO}_{4}(3 \times 30 \mathrm{~mL})$, and saturated aqueous $\mathrm{CuSO}_{4}(3 \times 30 \mathrm{~mL})$, dried over $\mathrm{Na}_{2} \mathrm{SO}_{4}$, and concentrated in vacuo. The resulting oil was purified by flash chromatography (1:9 EtOAc/hexanes) followed by a second flash chromatography (19:1 benzene/EtOAc) to afford the product (as a 1:1 mixture of anomers) as a colorless oil $(0.244 \mathrm{~g}, 70 \%):{ }^{3}{ }^{1} \mathrm{H}$ NMR (500 $\left.\mathrm{MHz}, \mathrm{CDCl}_{3}\right) \delta 7.38-7.18(\mathrm{~m}, 10 \mathrm{H}), 6.02(\mathrm{~d}, J=2.1,1 \mathrm{H}), 5.71(\mathrm{dd}, J=8.9,2.7,1 \mathrm{H}), 4.61(\mathrm{~m}$, 2H), $4.54(\mathrm{~m}, 2 \mathrm{H}), 4.07(\mathrm{dd}, J=11.6,4.3,1 \mathrm{H}), 3.85(\mathrm{dd}, J=10.9,4.9,1 \mathrm{H}), 3.56(\mathrm{t}, J=10.7$, $1 \mathrm{H}), 3.40(\mathrm{dd}, J=11.5,8.8,1 \mathrm{H}), 3.14(\mathrm{~m}, 2 \mathrm{H}), 2.08(\mathrm{~s}, 3 \mathrm{H}), 2.07(\mathrm{~s}, 3 \mathrm{H}), 1.99(\mathrm{~m}, 2 \mathrm{H}), 1.84(\mathrm{~m}$, $2 \mathrm{H}), 1.50(\mathrm{~m}, 1 \mathrm{H}), 1.40(\mathrm{~m}, 1 \mathrm{H}), 1.09(\mathrm{~d}, J=6.6,3 \mathrm{H}), 1.05(\mathrm{~d}, J=6.5,3 \mathrm{H}) ;{ }^{13} \mathrm{C}$ NMR $(125$ $\left.\mathrm{MHz}, \mathrm{CDCl}_{3}\right) \delta 169.8,169.4,138.23,138.17,128.7,128.4,128.2,128.0,127.8,127.7,93.6$, 91.0, 78.6, 78.0, 72.5, 72.3, 66.4, 63.2, 36.2, 36.0, 33.9, 30.9, 21.1, 18.2, 17.9; IR (thin film) 2928, 1749, 1235, 1075, 929, $699 \mathrm{~cm}^{-1}$; HRMS (CI/isobutane) $m / z$ calcd for $\mathrm{C}_{13} \mathrm{H}_{16} \mathrm{O}_{2}(\mathrm{M}-$ OAc) ${ }^{+}$204.1150, found 204.1147.

\section{Allylation Reactions}

Typical Allylation Procedure: Allyltrimethylsilane (4.0 equiv) was added to a solution of acetate $(0.15 \mathrm{M})$ in $\mathrm{CH}_{2} \mathrm{Cl}_{2}$, and the mixture was cooled to $-78{ }^{\circ} \mathrm{C}$ and treated with the Lewis acid (1.2 equiv). The mixture was allowed to warm to $23{ }^{\circ} \mathrm{C}$ and quenched with saturated aqueous $\mathrm{Na}_{2} \mathrm{HPO}_{4}(1 \mathrm{~mL}$ per mmol of acetate). The layers were separated, and the aqueous layer was extracted three times with $\mathrm{CH}_{2} \mathrm{Cl}_{2}$ ( $1 \mathrm{~mL}$ per mmol of acetate), dried over $\mathrm{Na}_{2} \mathrm{SO}_{4}$, and concentrated in vacuo. The unpurified product ratios were determined using GC and confirmed using GCMS. The reported yields are of purified material. 


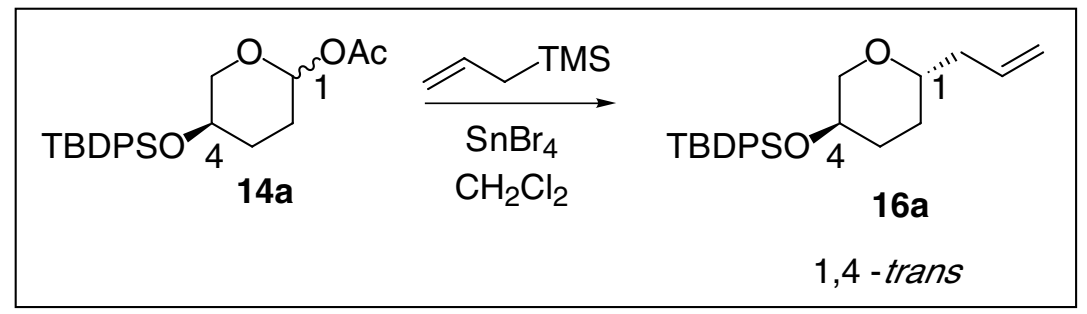

trans-1-Allyl-4-(tert-butyldiphenylsilyloxy)tetrahydropyran (16a): Under standard allylation conditions using $\mathrm{SnBr}_{4}$ as a Lewis acid, 4-(tertbutyldiphenylsilyloxy)tetrahydropyran-1-acetate $(0.109 \mathrm{~g}, 0.273 \mathrm{mmol})$ afforded the product $(0.103 \mathrm{~g}, 99 \%)$ as a 94:6 trans:cis mixture of isomers. Under standard allylation conditions using $\mathrm{BF}_{3} \cdot \mathrm{OEt}_{2}$ as a Lewis acid, 4-(tert-butyldiphenylsilyloxy)tetrahydropyran-1-acetate $(0.040$ $\mathrm{g}, 1.0 \mathrm{mmol})$ afforded the product $(0.034 \mathrm{~g}, 89 \%)$ as an 83:17 trans:cis mixture of isomers. The oil was purified by flash chromatography (1:99 to 1:9 EtOAc/hexanes): $\mathrm{GC} t_{\mathrm{R}}$ (major) 10.8 min, $t_{\mathrm{R}}$ (minor) $10.4 \mathrm{~min}\left(160^{\circ} \mathrm{C}\right.$ for $1 \mathrm{~min}, 10^{\circ} / \mathrm{min}$ to $\left.250^{\circ} \mathrm{C}\right) ; 1,4$-trans isomer (major): ${ }^{1} \mathrm{H}$ NMR $\left(500 \mathrm{MHz}, \mathrm{C}_{6} \mathrm{D}_{6}\right) \delta$ 7.75-7.70 (m, 4H), 7.23-7.15 (m, 6H), $5.79(\mathrm{~m}, 1 \mathrm{H}), 4.99-4.94(\mathrm{~m}, 2 \mathrm{H})$, $4.05(\mathrm{ddd}, J=10.7,4.9,2.3,1 \mathrm{H}), 3.80(\mathrm{~m}, 1 \mathrm{H}), 3.23(\mathrm{t}, J=10.3,1 \mathrm{H}), 3.04(\mathrm{~m}, 1 \mathrm{H}), 2.17(\mathrm{~m}$, 1H), $1.97(\mathrm{~m}, 1 \mathrm{H}), 1.87(\mathrm{~m}, 1 \mathrm{H}), 1.41(\mathrm{tdd}, J=13.0,10.7,4.2,1 \mathrm{H}), 1.23(\mathrm{~m}, 1 \mathrm{H}), 1.14(\mathrm{~s}, 9 \mathrm{H})$, $0.92(\operatorname{tdd}, J=13.6,11.1,3.9,1 \mathrm{H}) ;{ }^{13} \mathrm{C} \mathrm{NMR}\left(125 \mathrm{MHz}, \mathrm{C}_{6} \mathrm{D}_{6}\right) \delta 135.70,135.65,134.9,134.2$, $134.0,129.7,129.6,127.61,127.55,116.7,76.7,72.9,67.6,40.2,33.2,30.2,26.9$, 19.1; IR (thin film) 3071, 2933, 1428, $702 \mathrm{~cm}^{-1}$; HRMS (CI/isobutane) $m / z$ calcd for $\mathrm{C}_{24} \mathrm{H}_{31} \mathrm{O}_{2} \mathrm{Si}(\mathrm{M}-\mathrm{H})^{+}$ 379.2093, found 379.2080. Anal. Calcd for $\mathrm{C}_{24} \mathrm{H}_{32} \mathrm{O}_{2} \mathrm{Si}$ : C, 75.74; H, 8.47. Found: C, 75.60; H, 8.46.

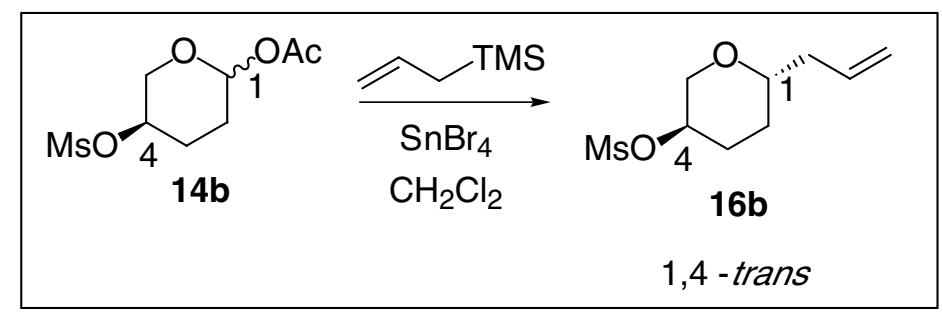


trans-1-Allyl-4-methanesulfoxytetrahydropyran (16b): Under standard allylation conditions using $\mathrm{SnBr}_{4}$ as a Lewis acid, 4-methanesulfoxytetrahydropyran-1-acetate $(0.115 \mathrm{~g}$, $0.483 \mathrm{mmol})$ afforded the product $(0.081 \mathrm{~g}, 76 \%)$ as a $96: 4$ trans:cis mixture of isomers. Under standard allylation conditions using $\mathrm{BF}_{3} \cdot \mathrm{OEt}_{2}$ as a Lewis acid, 4methanesulfoxytetrahydropyran-1-acetate $(0.116 \mathrm{~g}, 0.487 \mathrm{mmol})$ afforded the product $(0.075 \mathrm{~g}$, 70\%) as a 93:7 trans:cis mixture of isomers. The oil was purified by flash chromatography (1:9 to $1: 2 \mathrm{EtOAc} /$ hexanes): $\mathrm{GC} t_{\mathrm{R}}$ (major) $12.6 \mathrm{~min}, t_{\mathrm{R}}$ (minor) $12.4 \mathrm{~min}\left(50{ }^{\circ} \mathrm{C}\right.$ for $1 \mathrm{~min}, 10^{\circ} / \mathrm{min}$ to $\left.250{ }^{\circ} \mathrm{C}\right)$; 1,4-trans isomer (major): ${ }^{1} \mathrm{H}$ NMR (500 MHz, $\left.\mathrm{C}_{6} \mathrm{D}_{6}\right) \delta 5.73(\mathrm{~m}, 1 \mathrm{H}), 5.00-4.96$ $(\mathrm{m}, 2 \mathrm{H}), 4.42(\mathrm{tt}, J=10.3,5.2,1 \mathrm{H}), 4.03(\mathrm{ddd}, J=10.7,5.0,2.3,1 \mathrm{H}), 3.03(\mathrm{t}, J=10.5,1 \mathrm{H}), 2.84$ (m, 1H), $2.12(\mathrm{~m}, 1 \mathrm{H}), 2.08(\mathrm{~s}, 3 \mathrm{H}), 1.97-1.89(\mathrm{~m}, 2 \mathrm{H}), 1.27(\mathrm{tdd}, J=12.7,11.4,4.4,1 \mathrm{H}), 1.16$ $(\mathrm{m}, 1 \mathrm{H}), 0.94(\mathrm{tdd}, J=13.5,11.0,4.0,1 \mathrm{H}) ;{ }^{13} \mathrm{C} \mathrm{NMR}\left(125 \mathrm{MHz}, \mathrm{C}_{6} \mathrm{D}_{6}\right) \delta 134.5,116.6,76.4$, 74.6, 69.5, 40.1, 37.6, 30.5, 29.9; IR (thin film) 3021, 1359, 1176, 1097, $858 \mathrm{~cm}^{-1}$; HRMS (CI/isobutane) $m / z$ calcd for $\mathrm{C}_{9} \mathrm{H}_{17} \mathrm{O}_{4} \mathrm{~S}(\mathrm{M}+\mathrm{H})^{+} 221.0847$, found 221.0852

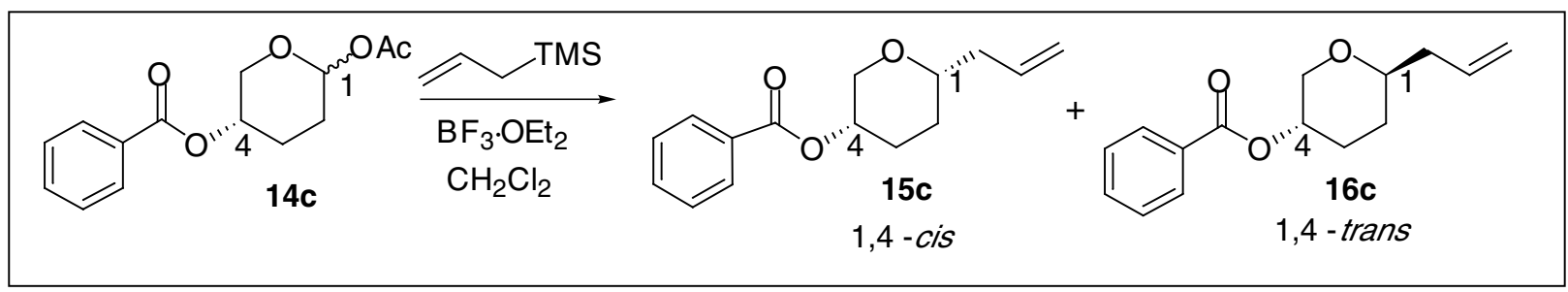

1-Allyl-4-benzoyloxytetrahydropyran (16c): Under standard allylation conditions using $\mathrm{BF}_{3} \cdot \mathrm{OEt}_{2}$ as a Lewis acid, 4-benzoyloxytetrahydropyran-1-acetate $(0.100 \mathrm{~g}, 0.378 \mathrm{mmol})$ afforded the product $(0.077 \mathrm{~g}, 83 \%)$ as an 93:7 trans:cis mixture of isomers. The oil was purified by flash column chromatography (hexanes to 1:9 EtOAc/hexanes) to afford the 1,4trans product as a colorless oil): ${ }^{1} \mathrm{H}$ NMR $\left(500 \mathrm{MHz}, \mathrm{C}_{6} \mathrm{D}_{6}\right) \delta 8.11(\mathrm{~m}, 2 \mathrm{H}), 7.33-7.06(\mathrm{~m}$, $3 \mathrm{H}), 5.85(\mathrm{~m}, 1 \mathrm{H}), 5.12(\mathrm{tt}, J=10.5,5.4,1 \mathrm{H}), 5.04(\mathrm{~m}, 2 \mathrm{H}), 4.18(\mathrm{ddd}, J=10.6,4.9,2.2,1 \mathrm{H})$, $3.20(\mathrm{t}, J=10.4,1 \mathrm{H}), 3.06(\mathrm{~m}, 1 \mathrm{H}), 2.23(\mathrm{~m}, 1 \mathrm{H}), 2.04(\mathrm{~m}, 2 \mathrm{H}) 1.33(\mathrm{~m}, 2 \mathrm{H}), 1.15(\mathrm{~m}, 1 \mathrm{H}) ;{ }^{13} \mathrm{C}$ 
NMR $\left(125 \mathrm{MHz}, \mathrm{CDCl}_{3}\right) \delta 165.4,134.3,132.7,129.9,129.3,128.0,116.7,76.7,69.2,68.5$, 39.7, 29.5, 29.0; IR (thin film) 2943, 2853, 1721, 1452, 1272, $1098 \mathrm{~cm}^{-1}$; HRMS (EI/GCMS) $m$ / $z$ calcd for $\mathrm{C}_{15} \mathrm{H}_{18} \mathrm{O}_{3}(\mathrm{M})^{+}$246.1256, found 246.1256. Anal. Calcd for $\mathrm{C}_{15} \mathrm{H}_{18} \mathrm{O}_{3}: \mathrm{C}, 73.15 ; \mathrm{H}$, 7.37. Found: C, 73.34; H, 7.59.

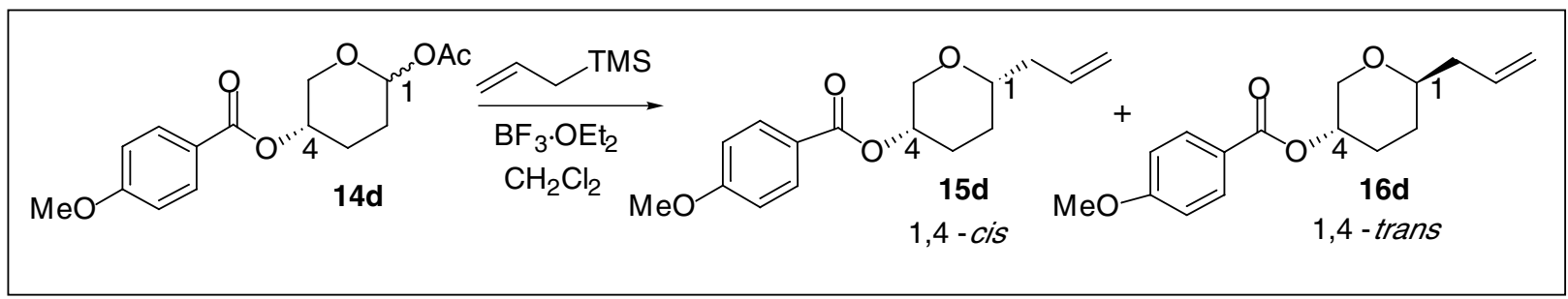

1-allyl-4-( $p$-anisoyloxy)tetrahydropyran (16d): Under standard allylation conditions using $\mathrm{BF}_{3} \cdot \mathrm{OEt}_{2}$ as a Lewis acid, 4-(p-anisoyloxy)tetrahydropyran-1-acetate $(0.083 \mathrm{~g}, 0.282 \mathrm{mmol})$ afforded the product as a 96:4 trans:cis mixture of isomers. The oil was purified by flash column chromatography (hexanes to 1:9 EtOAc/hexanes) to afford the 1,4-trans product as a colorless oil (0.065 g, 85\%): ${ }^{1} \mathrm{H}$ NMR (500 MHz, $\left.\mathrm{C}_{6} \mathrm{D}_{6}\right) \delta 8.13(\mathrm{~m}, 2 \mathrm{H}), 6.67(\mathrm{~m}, 2 \mathrm{H}), 5.84$ (m, 1H), $5.14(\mathrm{~m}, 1 \mathrm{H}), 5.03(\mathrm{~m}, 2 \mathrm{H}), 4.23(\mathrm{ddd}, J=10.5,4.9,2.1,1 \mathrm{H}), 3.23(\mathrm{t}, J=10.4,1 \mathrm{H})$, $3.20(\mathrm{~s}, 3 \mathrm{H}), 3.07(\mathrm{~m}, 1 \mathrm{H}), 2.27(\mathrm{~m}, 1 \mathrm{H}), 2.08(\mathrm{~m}, 2 \mathrm{H}), 1.31(\mathrm{~m}, 2 \mathrm{H}) 1.19(\mathrm{~m}, 1 \mathrm{H}) ;{ }^{13} \mathrm{C}$ NMR $\left(125 \mathrm{MHz}, \mathrm{CDCl}_{3}\right) \delta 165.5,163.4,135.7,131.6,122.6,117.0,113.6,77.0,69.6,68.4,55.4$, 40.1, 29.8, 29.3; IR (thin film) 2944, 1714, 1606, 1511, $1168 \mathrm{~cm}^{-1}$; HRMS (EI/GCMS) $\mathrm{m} / z$ calcd for $\mathrm{C}_{16} \mathrm{H}_{20} \mathrm{O}_{4}(\mathrm{M})^{+}$235.0970, found 235.0972. Anal. Calcd for $\mathrm{C}_{16} \mathrm{H}_{20} \mathrm{O}_{4}$ : C, 69.54; $\mathrm{H}$, 7.30. Found: C, 69.47; H, 7.46.

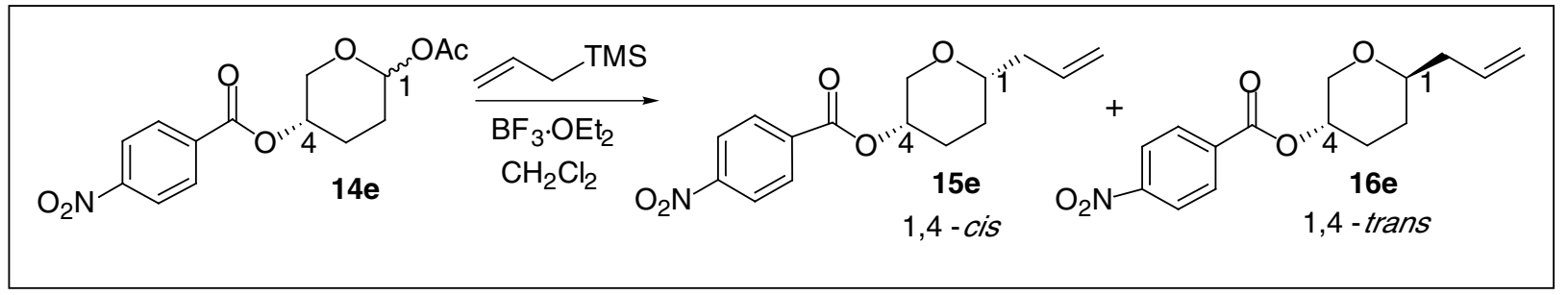


1-Allyl-4-(p-nitrobenzoyloxy)tetrahydropyran. (16e): Under standard allylation conditions using $\mathrm{BF}_{3} \cdot \mathrm{OEt}_{2}$ as a Lewis acid, 4-(p-nitrobenzoyloxy)tetrahydropyran-1-acetate $(0.047 \mathrm{~g}, 0.15$ mmol) afforded the product $(0.077 \mathrm{~g}, 83 \%)$ as an 81:19 trans:cis mixture of isomers. The oil was purified by flash column chromatography (hexanes to 1:9 EtOAc/hexanes) to afford the 1,4-trans product as a colorless oil $(0.040 \mathrm{~g}, 90 \%):{ }^{1} \mathrm{H} \mathrm{NMR}\left(500 \mathrm{MHz}, \mathrm{CDCl}_{3}\right) \delta 7.70(\mathrm{~m}$, 4H), $5.84(\mathrm{~m}, 1 \mathrm{H}), 5.07(\mathrm{~m}, 2 \mathrm{H}), 4.97(\mathrm{~m}, 1 \mathrm{H}), 4.11(\mathrm{ddd}, J=10.5,4.9,2.3,1 \mathrm{H}), 3.16(\mathrm{t}, J=$ 10.4, 1H), $3.07(\mathrm{~m}, 1 \mathrm{H}), 2.28(\mathrm{~m}, 1 \mathrm{H}), 2.07(\mathrm{~m}, 1 \mathrm{H}), 1.99(\mathrm{~m}, 1 \mathrm{H}), 1.35-1.29(\mathrm{~m}, 2 \mathrm{H}), 1.15(\mathrm{~m}$, $1 \mathrm{H}) ;{ }^{13} \mathrm{C} \mathrm{NMR}\left(125 \mathrm{MHz}, \mathrm{CDCl}_{3}\right) \delta 164.3,151.0,136.0,135.0,131.2,124.0,117.6,77.4,70.3$, 69.6, 40.4, 30.1, 29.6; HRMS (EI/GCMS) $m / z$ calcd for $\mathrm{C}_{12} \mathrm{H}_{11} \mathrm{NO}_{5}\left(\mathrm{M}-\mathrm{C}_{3} \mathrm{H}_{6}\right)^{+} 250.0715$, found 250.0711. Anal. Calcd for $\mathrm{C}_{15} \mathrm{H}_{17} \mathrm{NO}_{5}: \mathrm{C}, 61.85 ; \mathrm{H}, 5.88 ; \mathrm{N}, 4.81$. Found: $\mathrm{C}, 61.98 ; \mathrm{H}$, $6.02 ; \mathrm{N}, 4.70$.

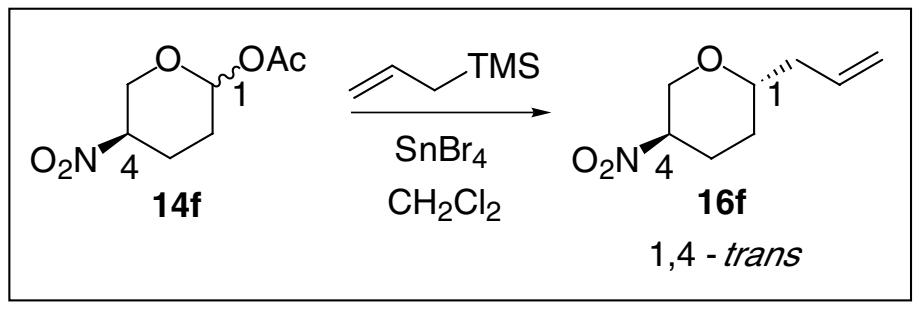

trans-1-Allyl-4-nitrotetrahydropyran (16f): Under standard allylation conditions using $\mathrm{SnBr}_{4}$ as a Lewis acid, 4-nitrotetrahydropyran1-1-acetate $(0.364 \mathrm{~g}, 1.92 \mathrm{mmol})$ afforded the product $(0.299 \mathrm{~g}, 91 \%)$ as a 85:15 trans:cis mixture of isomers. Under standard allylation conditions using $\mathrm{BF}_{3} \cdot \mathrm{OEt}_{2}$ as a Lewis acid, 4-nitrotetrahydropyran1-1-acetate (0.094 $\mathrm{g}, 0.50$ mmol) afforded the product $(0.075 \mathrm{~g}, 88 \%)$ as an 80:20 trans:cis mixture of isomers. The oil was purified by flash chromatography (1:99 to $1: 4 \mathrm{Et}_{2} \mathrm{O} /$ pentane): $\mathrm{GC} t_{\mathrm{R}}$ (major) 8.5 min, $t_{\mathrm{R}}$ (minor) $9.2 \min \left(50{ }^{\circ} \mathrm{C}\right.$ for $1 \mathrm{~min}, 10^{\circ} / \mathrm{min}$ to $\left.250{ }^{\circ} \mathrm{C}\right)$; 1,4-trans (major): ${ }^{1} \mathrm{H}$ NMR (500 $\left.\mathrm{MHz}, \mathrm{CDCl}_{3}\right) \delta 5.80(\mathrm{~m}, 1 \mathrm{H}), 5.12-5.07(\mathrm{~m}, 2 \mathrm{H}), 4.51(\mathrm{ddt}, J=11.9,10.5,4.5,1 \mathrm{H}), 4.38(\mathrm{ddd}$, $J=10.9,4.6,2.4,1 \mathrm{H}), 3.66(\mathrm{t}, J=10.7,1 \mathrm{H}), 3.39(\mathrm{~m}, 1 \mathrm{H}), 2.48(\mathrm{~m}, 1 \mathrm{H}), 2.32(\mathrm{~m}, 1 \mathrm{H}), 2.21(\mathrm{~m}$ 
1H), $2.07(\mathrm{qd}, J=12.7,4.4,1 \mathrm{H}), 1.88(\mathrm{ddt}, J=13.9,4.8,2.4,1 \mathrm{H}), 1.44(\mathrm{tdd}, J=13.6,11.2,4.0$, $1 \mathrm{H}) ;{ }^{13} \mathrm{C}$ NMR (125 MHz, $\left.\mathrm{CDCl}_{3}\right) \delta 133.9,117.4,79.9,76.7,68.6,39.7,29.2$, 28.3; IR (thin film) $3078,1546,1351,1091,768 \mathrm{~cm}^{-1}$; HRMS (CI/isobutane) $\mathrm{m} / z$ calcd for $\mathrm{C}_{5} \mathrm{H}_{8} \mathrm{NO}_{3}(\mathrm{M}-$ $\left.\mathrm{C}_{3} \mathrm{H}_{5}\right)^{+}$130.0504, found 130.0501. Anal. Calcd for $\mathrm{C}_{8} \mathrm{H}_{13} \mathrm{NO}_{3}$ : C, 56.13; H, 7.65; N, 8.18. Found: C, 56.04; H, 7.82; N, 8.21.

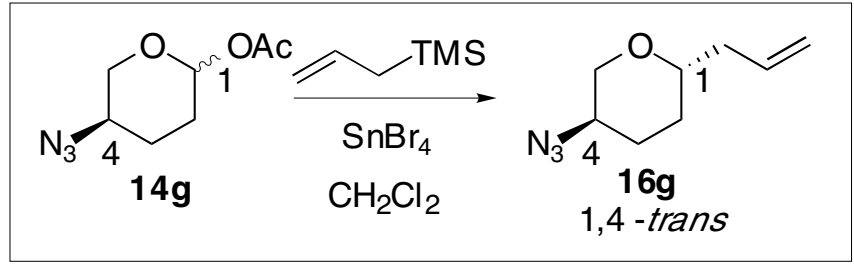

trans-1-Allyl-4-azidotetrahydropyran (16g): Under standard allylation conditions using $\mathrm{SnBr}_{4}$ as a Lewis acid, 4-azidotetrahydropyran-1-acetate $(0.095 \mathrm{~g}, 0.51 \mathrm{mmol})$ afforded the product $(0.081 \mathrm{~g}, 95 \%)$ as a $88: 12$ trans:cis mixture of isomers. Under standard allylation conditions using $\mathrm{BF}_{3} \cdot \mathrm{OEt}_{2}$ as a Lewis acid, 4-azidotetrahydropyran-1-acetate $(0.095 \mathrm{~g}, 0.51$ mmol) afforded the product $(0.081 \mathrm{~g}, 95 \%)$ as an 78:22 trans:cis mixture of isomers. The oil was purified by flash chromatography (99:1 to $4: 1$ pentane/Et $2 \mathrm{O}$ ): $\mathrm{GC} t_{\mathrm{R}}$ (major) $7.6 \mathrm{~min}$, $t_{\mathrm{R}}$ (minor) $8.0 \mathrm{~min}\left(50{ }^{\circ} \mathrm{C}\right.$ for $1 \mathrm{~min}, 10^{\circ} / \mathrm{min}$ to $\left.250{ }^{\circ} \mathrm{C}\right)$; 1,4 -trans isomer (major): ${ }^{1} \mathrm{H}$ NMR $\left(500 \mathrm{MHz}, \mathrm{CDCl}_{3}\right) \delta 5.80(\mathrm{~m}, 1 \mathrm{H}), 5.11-5.05(\mathrm{~m}, 2 \mathrm{H}), 4.01(\mathrm{ddd}, J=11.0,4.7,2.4,1 \mathrm{H}), 3.41$ (tt, $J=11.0,4.7,1 \mathrm{H}), 3.30(\mathrm{~m}, 1 \mathrm{H}), 3.17(\mathrm{t}, J=10.8,1 \mathrm{H}), 2.29(\mathrm{~m}, 1 \mathrm{H}), 2.23-2.15(\mathrm{~m}, 2 \mathrm{H}), 1.78$ (m, $1 \mathrm{H}), 1.51(\operatorname{tdd}, J=12.8,11.5,4.0,1 \mathrm{H}), 1.39$ (tdd, $J=13.4,10.9,3.7,1 \mathrm{H}) ;{ }^{13} \mathrm{C}$ NMR $(125$ $\left.\mathrm{MHz}, \mathrm{CDCl}_{3}\right) \delta 134.3,117.0,76.7,70.2,56.1,40.0,29.8,29.0 ;$ IR (thin film) 3077, 2850, 2104, 1271, 1119, $916 \mathrm{~cm}^{-1}$; HRMS (Cl/isobutane) $\mathrm{m} / z$ calcd for $\mathrm{C}_{8} \mathrm{H}_{13} \mathrm{~N}_{3} \mathrm{O}(\mathrm{M})^{+} 167.1059$, found 167.1060 . 


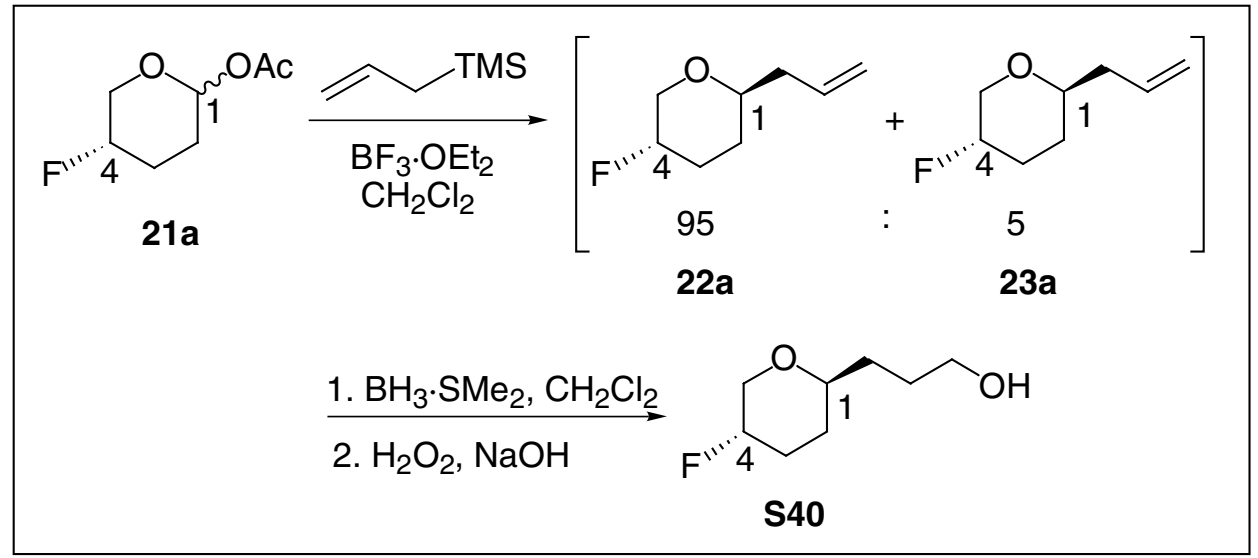

trans-1-(3-Hydroxypropyl)-4-fluorotetrahydropyran (S40): Allyltrimethylsilane (0.053 g, $0.466 \mathrm{mmol})$ was added to a solution of 4-fluorotetrahydropyran-1-acetate $(0.018 \mathrm{~g}, 0.117$ $\mathrm{mmol})$ in $\mathrm{CD}_{2} \mathrm{Cl}_{2}(1 \mathrm{~mL})$, and the mixture was cooled to $-78{ }^{\circ} \mathrm{C}$ and treated with $\mathrm{BF}_{3} \cdot \mathrm{OEt}_{2}$ $(0.018 \mathrm{~mL}, 0.140 \mathrm{mmol})$. The reaction afforded a 95:5 trans:cis mixture of anomers, as determined based on GC and ${ }^{1} \mathrm{H}$ NMR. After $3 \mathrm{~h}$, the mixture was allowed to warm to $23{ }^{\circ} \mathrm{C}$ and the reaction was monitored by ${ }^{1} \mathrm{H}$ NMR spectroscopy: ${ }^{1} \mathrm{H}$ NMR $\left(500 \mathrm{MHz}, \mathrm{CD}_{2} \mathrm{Cl}_{2}\right) \delta$ $4.70-4.45$ (dtt, $J=48.8,10.3,5.3,1 \mathrm{H}), 4.15$ (ddt, $J=10.7,5.1,2.5,1 \mathrm{H}), 3.43(\mathrm{dtd}, J=10.5,6.0$, $1.81 \mathrm{H}), 3.37(\mathrm{td}, J=10.1,3.7,1 \mathrm{H})$. The solution was cooled to $0{ }^{\circ} \mathrm{C}$ and $10 \mathrm{M} \mathrm{BH}_{3} \cdot \mathrm{SMe}_{2}$ $(0.005 \mathrm{~mL}, 0.048 \mathrm{mmol})$ was added. The reaction mixture was stirred at $23{ }^{\circ} \mathrm{C}$ for $72 \mathrm{~h}$ and cooled to $0{ }^{\circ} \mathrm{C}$ before $10 \% \mathrm{NaOH}(0.055 \mathrm{~mL})$ followed by $30 \% \mathrm{H}_{2} \mathrm{O}_{2}(0.028 \mathrm{~mL})$ were added. The reaction mixture was allowed to warm to $23{ }^{\circ} \mathrm{C}$ and stirred for $48 \mathrm{~h}$. Solid $\mathrm{K}_{2} \mathrm{CO}_{3}(0.049 \mathrm{~g})$ was added, and the mixture was allowed to stir for an additional $2 \mathrm{~h}$. The $\mathrm{CD}_{2} \mathrm{Cl}_{2}$ layer was washed with $\mathrm{H}_{2} \mathrm{O}(10 \mathrm{~mL})$ and the aqueous layer was extracted with $\mathrm{CH}_{2} \mathrm{Cl}_{2}(3 \times 1 \mathrm{~mL})$. The combined organic phases were filtered through $\mathrm{Na}_{2} \mathrm{SO}_{4}$, and concentrated in vacuo. The resulting yellow oil was purified by flash column chromatography $\left(1: 9 \mathrm{Et}_{2} \mathrm{O} /\right.$ pentane to $3: 2$ $\mathrm{Et}_{2} \mathrm{O} /$ pentane) to afford the 1,4-trans product as a colorless oil $(0.009 \mathrm{~g}, 45 \%):{ }^{1} \mathrm{H}$ NMR (500 $\left.\mathrm{MHz}, \mathrm{CDCl}_{3}\right) \delta 4.70-4.45(\mathrm{dtt}, J=48.8,10.3,5.3,1 \mathrm{H}), 4.12(\mathrm{~m}, 1 \mathrm{H}), 3.65(\mathrm{bm}, 2 \mathrm{H}), 3.31-3.26$ (td, $J=10.4,3.3,1 \mathrm{H}$ and $\mathrm{m}, 1 \mathrm{H}), 2.23(\mathrm{~m}, 1 \mathrm{H}), 1.94(\mathrm{bs}, 1 \mathrm{H}), 1.68-1.64(\mathrm{~m}, 1 \mathrm{H}), 1.60-1.55(\mathrm{~m}$, $5 \mathrm{H}), 1.43(\mathrm{~m}, 1 \mathrm{H}) ;{ }^{13} \mathrm{C} \mathrm{NMR}\left(125 \mathrm{MHz}, \mathrm{CDCl}_{3}\right) \delta 87.2(\mathrm{~d}, J=174), 69.6(\mathrm{~d}, J=$ 
23), 62.8, $32.0(\mathrm{~d}, J=2), 30.2,30.01(\mathrm{~d}, J=8), 29.95,29.2 ;{ }^{19} \mathrm{~F} \mathrm{NMR}\left(376 \mathrm{MHz}, \mathrm{CDCl}_{3}\right)$ -187.5 (m); IR (thin film) 3352, 2946, 2866, 1446, 1377, 1092, $1038 \mathrm{~cm}^{-1}$; HRMS (EI/GCMS) $m / z$ calcd for $\mathrm{C}_{8} \mathrm{H}_{14} \mathrm{O}_{2}(\mathrm{M}-\mathrm{HF})^{+}$142.0994, found 142.0991.
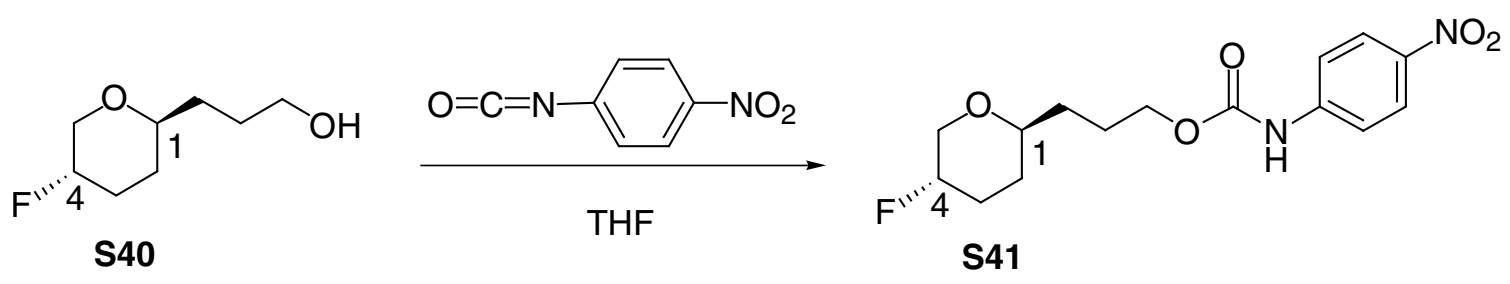

trans-(4-Nitrophenyl)carbamic acid-4-fluorotetrahydropyran (S41): To a solution of trans-1-(1-Propanol)-4-fluorotetrahydropyran $(0.026 \mathrm{~g}, 0.16 \mathrm{mmol})$ in $0.5 \mathrm{~mL}$ of THF at $0{ }^{\circ} \mathrm{C}$ was added 4-nitrophenyl isocyanate $(0.028 \mathrm{~g}, 0.17 \mathrm{mmol})$. The reaction mixture was allowed to warm to $23{ }^{\circ} \mathrm{C}$ and stirred for $24 \mathrm{~h}$. The layers were separated, and the aqueous layer was extracted with $\mathrm{Et}_{2} \mathrm{O}(3 \times 2 \mathrm{~mL})$, dried over $\mathrm{MgSO}_{4}$, filtered, and concentrated in vacuo. The resulting oil was purified by flash column chromatography (pentane to $1: 1 \mathrm{Et}_{2} \mathrm{O} /$ pentane) to afford the product as a white solid $(0.23 \mathrm{~g}, 44 \%)$ : ${ }^{1} \mathrm{H}$ NMR $\left(500 \mathrm{MHz}, \mathrm{C}_{6} \mathrm{D}_{6}\right) \delta 7.80(\mathrm{~m}, 2 \mathrm{H})$, 6.87 (m, 2H), 5.9 (bs, 1H), 4.35-4.19 (dtt, $J=48.8,10.4,5.2,1 \mathrm{H}), 4.00$ (m, 1H and t, $J=6.4$, 2H), $3.10(\mathrm{td}, J=10.3,3.4,1 \mathrm{H}), 2.78$ (dddd, $J=10.8,8.7,3.9,1.9,1 \mathrm{H}), 1.88(\mathrm{~m}, 1 \mathrm{H}), 1.67$ (m, $1 \mathrm{H}), 1.48(\mathrm{~m}, 1 \mathrm{H}), 1.35(\mathrm{~m}, 2 \mathrm{H}), 1.15(\mathrm{~m}, 2 \mathrm{H}), 0.93(\mathrm{~m}, 1 \mathrm{H}) ;{ }^{13} \mathrm{C}$ NMR $\left(125 \mathrm{MHz}, \mathrm{C}_{6} \mathrm{D}_{6}\right)$ $\delta 152.3,143.4,124.67,117.07,86.8$ (d, $J=175), 76.1,69.4$ (d, $J=28), 65.4,31.4$ (d, $J=$ 2), 30.0 (d, $J=18), 29.8$ (d, $\left.J=9.4), 25.2 ;{ }^{19} \mathrm{~F} \mathrm{NMR} \mathrm{(376} \mathrm{MHz,} \mathrm{CDCl}_{3}\right)-188.0(\mathrm{~m})$; IR (thin film) 3403, 2918, 1749, 1543, 1221, $1038 \mathrm{~cm}^{-1}$; HRMS (CI/GCMS) $\mathrm{m} / \mathrm{z}$ calcd for $\mathrm{C}_{15} \mathrm{H}_{19} \mathrm{FN}_{2} \mathrm{O}_{5}(\mathrm{M})^{+} 326.1278$, found 326.1268 . 


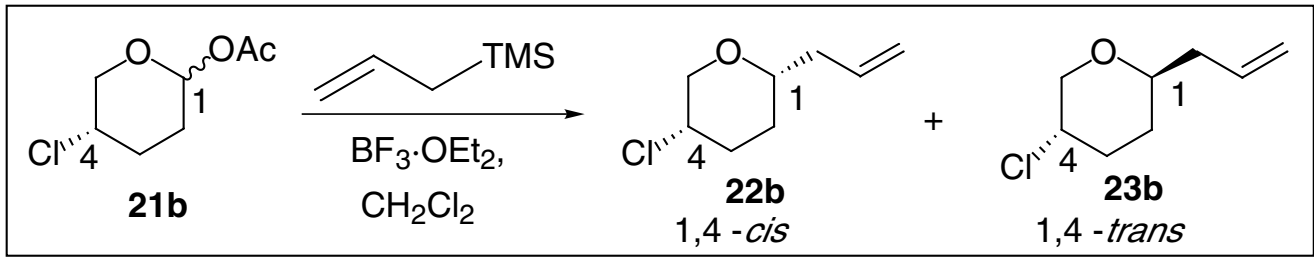

1-Allyl-4-chlorotetrahydropyran (22b, 23b): Under standard allylation conditions using $\mathrm{SnBr}_{4}$ as a Lewis acid, 4-chlorotetrahydropyran-1-acetate (1.02 g, $\left.5.71 \mathrm{mmol}\right)$ afforded $\mathbf{2 2 b}$, 23b $(0.828 \mathrm{~g}, 90 \%)$ as a separable 86:14 trans (23b):cis (22b) mixture of isomers. The oil was purified by flash chromatography (pentane to 1:49 to 1:9 $\mathrm{Et}_{2} \mathrm{O} /$ pentane): $\mathrm{GC} t_{\mathrm{R}}$ (major) 8.9 min, $t_{\mathrm{R}}$ (minor) $10.1 \mathrm{~min}\left(50{ }^{\circ} \mathrm{C}\right.$ for $\left.2 \mathrm{~min}, 5^{\circ} / \mathrm{min}\right)$; 1,4-trans isomer (major): ${ }^{1} \mathrm{H}$ NMR $(500 \mathrm{MHz}$, $\left.\mathrm{C}_{6} \mathrm{D}_{6}\right) \delta 5.73(\mathrm{~m}, 1 \mathrm{H}), 4.97(\mathrm{~m}, 2 \mathrm{H}), 3.97(\mathrm{ddd}, J=10.9,4.7,2.3,1 \mathrm{H}), 3.52(\mathrm{tt}, J=11.2,4.6$, $1 \mathrm{H}), 3.13(\mathrm{t}, J=10.8,1 \mathrm{H}), 2.88(\mathrm{~m}, 1 \mathrm{H}), 2.12(\mathrm{~m}, 1 \mathrm{H}), 1.96-1.85(\mathrm{~m}, 2 \mathrm{H}), 1.36(\mathrm{qd}, J=12.6$, $4.2,1 \mathrm{H}), 1.13(\mathrm{~m}, 1 \mathrm{H}), 0.93(\mathrm{qd}, J=12.7,3.8,1 \mathrm{H}) ;{ }^{13} \mathrm{C} \mathrm{NMR}\left(125 \mathrm{MHz}, \mathrm{C}_{6} \mathrm{D}_{6}\right) \delta 134.9,116.8$, 76.6, 73.1, 54.3, 40.3, 34.5, 31.7; IR (thin film) 2947, 1089, $765 \mathrm{~cm}^{-1}$; HRMS (CI/isobutane) $m$ / $z$ calcd for $\mathrm{C}_{8} \mathrm{H}_{12} \mathrm{ClO}(\mathrm{M}-\mathrm{H})^{+}$159.0577, found 159.0578. Anal. Calcd for $\mathrm{C}_{8} \mathrm{H}_{13} \mathrm{ClO}$ : C, 59.81; H, 8.16. Found: C, 59.97; H, 8.11; 1,4-cis isomer (minor): ${ }^{1} \mathrm{H} \mathrm{NMR}\left(500 \mathrm{MHz}, \mathrm{CDCl}_{3}\right)$ $\delta 5.85(\mathrm{~m}, 1 \mathrm{H}), 5.09(\mathrm{~m}, 2 \mathrm{H}), 4.17$ (br s, $1 \mathrm{H}), 4.02(\mathrm{dt}, J=12.7,2.3,1 \mathrm{H}), 3.77(\mathrm{dd}, J=12.7,1.8$, 1H), $3.39(\mathrm{~m}, 1 \mathrm{H}), 2.40(\mathrm{~m}, 1 \mathrm{H}), 2.24(\mathrm{~m}, 1 \mathrm{H}), 2.11(\mathrm{~m}, 1 \mathrm{H}), 1.99(\mathrm{~m}, 1 \mathrm{H}), 1.86(\mathrm{~m}, 1 \mathrm{H}), 1.48$ $(\mathrm{m}, 1 \mathrm{H}) ;{ }^{13} \mathrm{C} \mathrm{NMR}\left(125 \mathrm{MHz}, \mathrm{CDCl}_{3}\right) \delta 134.5,117.0,77.4,72.1,55.9,40.3,31.2,25.5 ; \mathrm{IR}$ (thin film) 2952, 1064, $886 \mathrm{~cm}^{-1}$; HRMS (CI/isobutane) $m / z$ calcd for $\mathrm{C}_{8} \mathrm{H}_{12} \mathrm{ClO}(\mathrm{M}-\mathrm{H})^{+}$ 159.0577, found 159.0578. Anal. Calcd for $\mathrm{C}_{8} \mathrm{H}_{13} \mathrm{OCl}$ : C, 59.81; H, 8.16. Found: C, 59.92; H, 8.11.

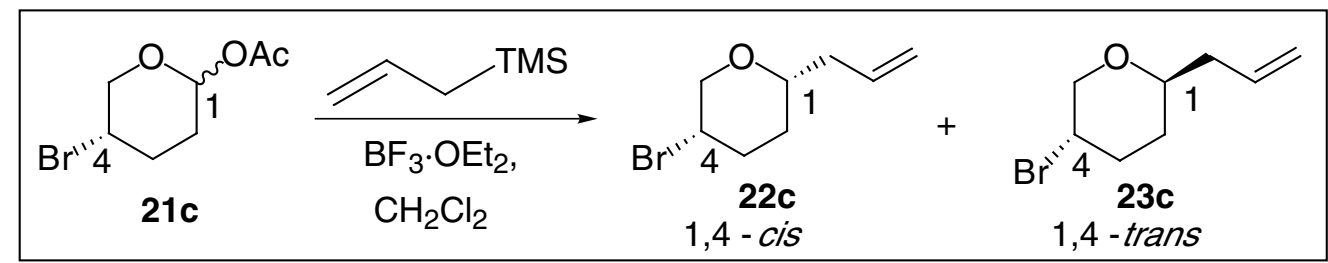


1-Allyl-4-bromotetrahydropyran (22c, 23c): Under standard allylation conditions using $\mathrm{SnBr}_{4}$ as a Lewis acid, 4-bromotetrahydropyran-1-acetate $(0.204 \mathrm{~g}, 0.928 \mathrm{mmol})$ afforded 22c, 23c $(0.168 \mathrm{~g}, 87 \%)$ as a separable $71: 29$ trans $(\mathbf{2 3 c})$ :cis (22c) mixture of isomers. Under standard allylation conditions using $\mathrm{BF}_{3} \cdot \mathrm{OEt}_{2}$ as a Lewis acid, 4-bromotetrahydropyran-1acetate $(0.207 \mathrm{~g}, 0.914 \mathrm{mmol})$ afforded $22 \mathrm{c}, 23 \mathrm{c}(0.164 \mathrm{~g}, 88 \%)$ as a separable $58: 42$ trans (23c):cis (22c) mixture of isomers. The oil was purified by flash chromatography (pentane to 1:49 to $1: 9 \mathrm{Et}_{2} \mathrm{O} /$ pentane): $\mathrm{GC} t_{\mathrm{R}}$ (major) $7.1 \mathrm{~min}, t_{\mathrm{R}}$ (minor) $7.8 \mathrm{~min}\left(100{ }^{\circ} \mathrm{C}\right.$ for $1 \mathrm{~min}, 10^{\circ}$ /min); 1,4-trans isomer (major): ${ }^{1} \mathrm{H}$ NMR (500 MHz, $\left.\mathrm{C}_{6} \mathrm{D}_{6}\right) \delta 5.72(\mathrm{~m}, 1 \mathrm{H}), 4.99-4.94(\mathrm{~m}$, 2H), $4.00(\mathrm{ddd}, J=11.0,4.6,2.3,1 \mathrm{H}), 3.61(\mathrm{tt}, J=11.4,4.6,1 \mathrm{H}), 3.25(\mathrm{t}, J=11.0,1 \mathrm{H}), 2.89(\mathrm{~m}$, $1 \mathrm{H}), 2.11(\mathrm{~m}, 1 \mathrm{H}), 1.96-1.89(\mathrm{~m}, 2 \mathrm{H}), 1.51(\mathrm{qd}, J=12.6,4.2,1 \mathrm{H}), 1.10(\mathrm{~m}, 1 \mathrm{H}), 0.95(\mathrm{tdd}, J=$ 13.4, 11.1, 3.8, 1H); ${ }^{13} \mathrm{C}$ NMR (125 MHz, $\left.\mathrm{C}_{6} \mathrm{D}_{6}\right) \delta 135.2,117.2,76.9,73.8,46.9,40.8,35.7$, 33.4; IR (thin film) 3076, 2850, 1642, 1083, $731 \mathrm{~cm}^{-1}$; HRMS (CI/isobutane) $m / z$ calcd for $\mathrm{C}_{5} \mathrm{H}_{8} \mathrm{BrO}\left(\mathrm{M}-\mathrm{C}_{3} \mathrm{H}_{5}\right)^{+}$162.9759, found 162.9756. Anal. Calcd for $\mathrm{C}_{8} \mathrm{H}_{13} \mathrm{BrO}$ : C, 46.85; $\mathrm{H}$, 6.39. Found: C, 46.58; H, 6.44. 1,4 -cis isomer (minor): ${ }^{1} \mathrm{H} \mathrm{NMR}\left(500 \mathrm{MHz}, \mathrm{CDCl}_{3}\right) \delta 5.85$ (m, 1H), 5.02-4.99 (m, 2H), $3.79(\mathrm{dt}, J=12.9,2.2,1 \mathrm{H}), 3.64(\mathrm{~d}, J=1.7,1 \mathrm{H}), 3.12(\mathrm{dd}, J=12.9$, 1.9, 1H), $2.95(\mathrm{~m}, 1 \mathrm{H}), 2.31(\mathrm{~m}, 1 \mathrm{H}), 2.06(\mathrm{~m}, 1 \mathrm{H}), 1.81-1.71(\mathrm{~m}, 2 \mathrm{H}), 1.27(\mathrm{~m}, 1 \mathrm{H}), 1.00(\mathrm{~m}$, $1 \mathrm{H}) ;{ }^{13} \mathrm{C} \mathrm{NMR}\left(125 \mathrm{MHz}, \mathrm{CDCl}_{3}\right) \delta 135.5,117.2,77.5,72.7,50.0,41.0,32.4,26.7$; IR (thin film) 3076, 2850, 1642, 1083, $731 \mathrm{~cm}^{-1}$; HRMS (CI/isobutane) $m / z$ calcd for $\mathrm{C}_{5} \mathrm{H}_{8} \mathrm{BrO}(\mathrm{M}-$ $\left.\mathrm{C}_{3} \mathrm{H}_{5}\right)^{+}$162.9759, found 162.9756. Anal. Calcd for $\mathrm{C}_{8} \mathrm{H}_{13} \mathrm{BrO}$ : C, 46.85; H, 6.39. Found: C, 47.10; H, 6.42.

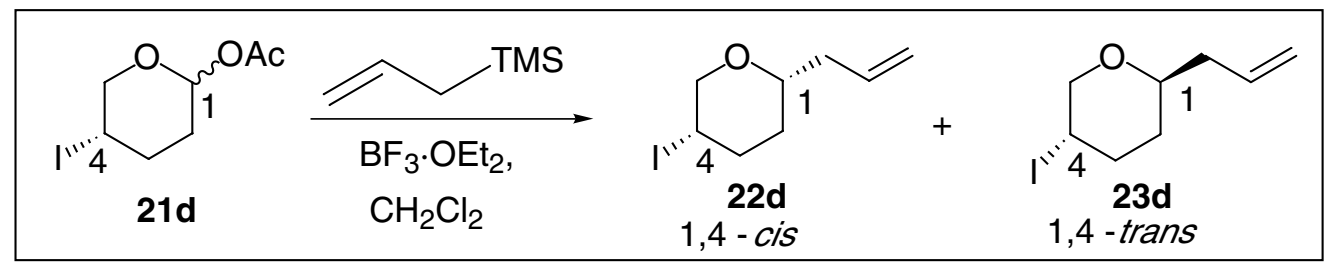


1-Allyl-4-iodotetrahydropyran (22d, 23d): Under standard allylation conditions using $\mathrm{BF}_{3} \cdot \mathrm{OEt}_{2}$ as a Lewis acid, 4-iodotetrahydropyran-1-acetate $(0.055 \mathrm{~g}, 0.20 \mathrm{mmol})$ afforded $\mathbf{2 2 d}$, 23d $(0.050 \mathrm{~g}, 97 \%)$ as a 2:1 1,4-cis:trans mixture of isomers. The oil was purified by flash chromatography (pentane to 1:25 $\mathrm{Et}_{2} \mathrm{O} /$ pentane) to afford the 1,4-cis product and a 2:1 trans:cis mixture of isomers:

1,4-cis isomer (22d): ${ }^{1} \mathrm{H}$ NMR $\left(500 \mathrm{MHz}, \mathrm{CDCl}_{3}\right) \delta 5.90-5.82(\mathrm{~m}, 1 \mathrm{H}), 5.14-5.07(\mathrm{~m}, 2 \mathrm{H})$, $4.48(\mathrm{~m}, 1 \mathrm{H}), 4.06(\mathrm{~d}, J=13.0,1 \mathrm{H}), 3.56(\mathrm{~d}, J=13.0,1 \mathrm{H}), 3.45-3.42(\mathrm{~m}, 1 \mathrm{H}), 2.46-2.40(\mathrm{~m}$, 1H), $2.30-2.24(\mathrm{~m}, 1 \mathrm{H}) 2.17-2.12(\mathrm{~m}, 1 \mathrm{H}) 1.91-1.74(\mathrm{~m}, 2 \mathrm{H}), 1.62-1.55(\mathrm{~m}, 1 \mathrm{H}) ;{ }^{13} \mathrm{C}$ NMR $\left(125 \mathrm{MHz}, \mathrm{CDCl}_{3}\right) \delta 135.0,117.5,77.9,74.5,40.6,34.0,30.0,28.2$; IR (thin film) 2944, 2841, 1434, 1278, 1112, $757 \mathrm{~cm}^{-1}$; HRMS (EI/GCMS) $m / z$ calcd for $\mathrm{C}_{8} \mathrm{H}_{13} \mathrm{IO}(\mathrm{M})^{+} 252.0011$. Submitted. Anal. Calcd for $\mathrm{C}_{8} \mathrm{H}_{13} \mathrm{IO}$ : C, 38.12; H, 5.20. Submitted.

1,4-cis and 1,4-trans isomers (22d, 23d): ${ }^{1} \mathrm{H} \mathrm{NMR}\left(500 \mathrm{MHz}, \mathrm{CDCl}_{3}\right) \delta 5.88-5.76(\mathrm{~m}, 2 \mathrm{H})$, 5.14-5.05 (m, 4H), $4.48(\mathrm{~m}, 1 \mathrm{H}), 4.14-4.05(\mathrm{~m}, 3 \mathrm{H}), 3.60(\mathrm{dd}, J=11.9,10.5,1 \mathrm{H}$ and $\mathrm{m}, 1 \mathrm{H}))$, 3.46-3.41 (m, 2H), 2.49-2.40 (m, 2H), 2.30-2.24 (m, 2H) 2.17-2.05 (m, 3H) 1.88-1.81 (m, $2 \mathrm{H}), 1.65(\mathrm{~m}, 1 \mathrm{H}) 1.60-1.50(\mathrm{~m}, 2 \mathrm{H}) ;{ }^{13} \mathrm{C} \mathrm{NMR}\left(125 \mathrm{MHz}, \mathrm{CDCl}_{3}\right) \delta 134.8,134.6,117.4$, 117.3, 77.7, 75.4, 74.3, 40.8, 40.4, 37.3, 35.0, 33.8, 29.9, 28.0, 25.2; IR (thin film) 2942, 2843, $1112,1075,917,757$; HRMS (EI/GCMS) $m / z$ calcd for $\mathrm{C}_{8} \mathrm{H}_{13} \mathrm{IO}(\mathrm{M})^{+} 252.0011$.

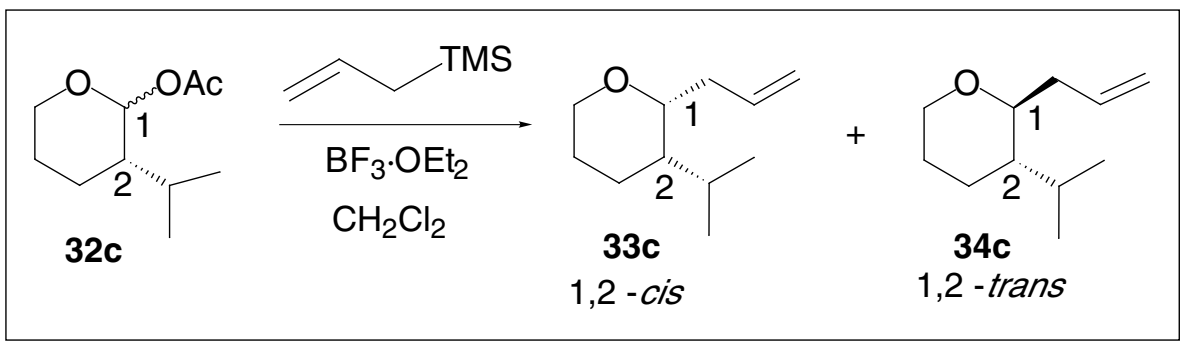

1-Allyl-2-isopropyltetrahydropyran (33c, 34c): Under standard allylation conditions using $\mathrm{BF}_{3} \cdot \mathrm{OEt}_{2}$ as the Lewis acid, 2-isopropyltetrahydropyran-1-acetate $(0.161 \mathrm{~g}, 0.866 \mathrm{mmol})$ afforded $1(0.064 \mathrm{~g}, 44 \%)$ as a 40:60 1,2-cis:trans mixture of isomers. The oil was purified by 
flash chromatography (pentane to $1: 9 \mathrm{Et}_{2} \mathrm{O} /$ pentane) to afford the 1,2-cis isomer $(0.080 \mathrm{~g})$ and the 1,2-trans isomer $(0.077 \mathrm{~g})$ :

1,2-cis (33c): ${ }^{1} \mathrm{H}$ NMR (500 MHz, $\left.\mathrm{CDCl}_{3}\right) \delta 5.95-5.88(\mathrm{~m}, 1 \mathrm{H}), 5.09-5.03(\mathrm{~m}, 2 \mathrm{H}), 3.86(\mathrm{~m}$, 1H), $3.33(\mathrm{~m}, 1 \mathrm{H}), 3.25(\mathrm{ddd}, J=9.5,7.8,3.0,1 \mathrm{H}), 2.45(\mathrm{~m}, 1 \mathrm{H}), 2.18(\mathrm{dt}, J=14.7,7.4,1 \mathrm{H})$, $1.90(\mathrm{~m}, 1 \mathrm{H}), 1.73(\mathrm{~m}, 1 \mathrm{H}), 1.59(\mathrm{~m}, 2 \mathrm{H}), 1.53(\mathrm{~m}, 2 \mathrm{H}), 0.89(\mathrm{~d}, J=6.9,3 \mathrm{H}), 0.77(\mathrm{~d}, J=6.9$, $3 \mathrm{H}) ;{ }^{13} \mathrm{C}$ NMR $\left(125 \mathrm{MHz}, \mathrm{CDCl}_{3}\right)$ $\delta 135.9,116.5,80.2,68.6,45.1,37.6,27.0,26.8,22.2,21.3,16.0$; IR (thin film) 2959, 2850, 1641, 1464, $1099 \mathrm{~cm}-1$; HRMS (EI/GCMS) $m / z$ calcd for $\mathrm{C}_{11} \mathrm{H}_{20} \mathrm{O}(\mathrm{M})+168.1514$, found 168.1515.

1,2-trans (34c): ${ }^{1} \mathrm{H}$ NMR (500 MHz, $\left.\mathrm{CDCl}_{3}\right) \delta 5.95-5.88(\mathrm{~m}, 1 \mathrm{H}), 5.09-5.03(\mathrm{~m}, 2 \mathrm{H}), 3.94$ $(\mathrm{dt}, J=11.3,3.9,1 \mathrm{H}), 3.58(\mathrm{~m}, 2 \mathrm{H}), 2.62(\mathrm{ddd}, J=14.9,11.3,7.4,1 \mathrm{H}), 2.03(\mathrm{~m}, 1 \mathrm{H}), 1.75(\mathrm{~m}$, $1 \mathrm{H}), 1.65(\mathrm{~m}, 2 \mathrm{H}), 1.40(\mathrm{~m}, 3 \mathrm{H}), 0.91(\mathrm{~d}, J=6.4,3 \mathrm{H}), 0.89(\mathrm{~d}, J=6.3,3 \mathrm{H}) ;{ }^{13} \mathrm{C}$ NMR $(125$ $\left.\mathrm{MHz}, \mathrm{CDCl}_{3}\right) \delta 136.3,116.3,75.8,60.8,45.7,30.0,29.2,26.3,22.9,21.2,21.0 ;$ IR (thin film) 2932, 2859, 1641, 1471, 1085, $910 \mathrm{~cm}^{-1}$; HRMS (EI/GCMS) $m / z$ calcd for $\mathrm{C}_{11} \mathrm{H}_{19} \mathrm{O}(\mathrm{M}-\mathrm{H})^{+}$ 167.1436, found 167.1437. Anal. Calcd for $\mathrm{C}_{11} \mathrm{H}_{20} \mathrm{O}: \mathrm{C}, 78.51 ; \mathrm{H}, 11.98$. Found: C, 78.38; $\mathrm{H}$, 12.05 .

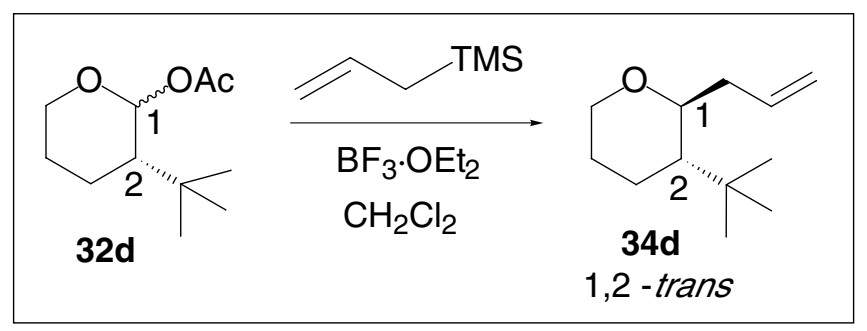

trans-1-Allyl-2-tert-butyltetrahydropyran (34d): Under standard allylation conditions using $\mathrm{BF}_{3} \cdot \mathrm{OEt}_{2}$ as a Lewis acid, 2-tert-butyltetrahydropyran-1-acetate $(0.374 \mathrm{~g}, 1.87 \mathrm{mmol})$ afforded $69(0.197 \mathrm{~g}, 58 \%)$ as a 1:99 1,2-cis:trans mixture of isomers. The oil was purified by 
flash chromatography (pentane to $1: 9 \mathrm{Et}_{2} \mathrm{O}$ /pentane) to afford the 1,2-trans isomer as a colorless oil:

1,2-trans-isomer (34d): ${ }^{1} \mathrm{H}$ NMR $\left(500 \mathrm{MHz}, \mathrm{CDCl}_{3}\right) \delta 5.93-5.88(\mathrm{~m}, 1 \mathrm{H})$, 5.09-5.03 (m, 2H), $3.86(\mathrm{dddd}, J=11.1,5.1,3.7,1.2,1 \mathrm{H}), 3.41(\mathrm{ddd}, J=8.6,7.4,4.0,1 \mathrm{H}), 3.37(\mathrm{td}, J=11.0$, 3.8, 1H), $2.48(\mathrm{~m}, 1 \mathrm{H}), 2.37(\mathrm{~m}, 1 \mathrm{H}), 1.84$ (dddd, $J=13.2,9.3,4.1,1.3,1 \mathrm{H}), 1.66(\mathrm{~m}, 1 \mathrm{H}), 1.54$ (m, 1H), $1.36(\mathrm{tdd}, J=11.1,8.6,4.2,1 \mathrm{H}), 1.19(\mathrm{ddd}, J=10.3,7.3,4.3,1 \mathrm{H}), 0.94(\mathrm{~s}, 9 \mathrm{H}) ;{ }^{13} \mathrm{C}$ NMR (125 MHz, $\left.\mathrm{CDCl}_{3}\right) \delta 136.8,116.3,79.4,66.2,48.4,40.2$, 33.1, 29.5, 26.2, 24.6; IR (thin film) 2958, 2870, $1112 \mathrm{~cm}^{-1}$; HRMS (EI/GCMS) $m / z$ calcd for $\mathrm{C}_{11} \mathrm{H}_{19} \mathrm{O}\left(\mathrm{M}-\mathrm{CH}_{3}\right)^{+}$ 167.1436, found 167.1432 .

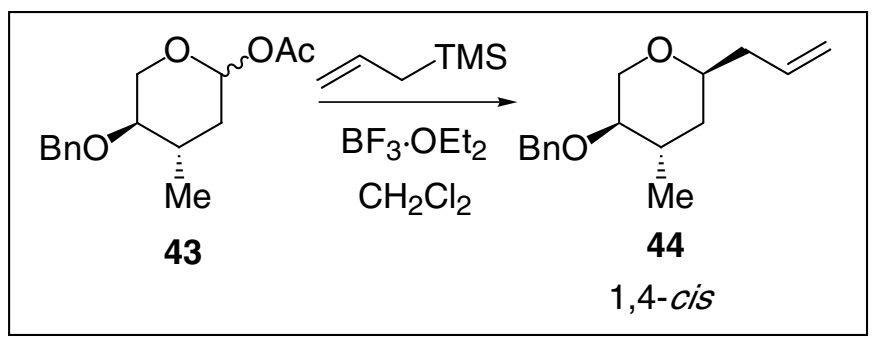

(2S,4S,5R)-2-allyl-5-benzyloxy-4-methyltetrahydropyran (44): Under standard allylation conditions using $\mathrm{BF}_{3} \cdot \mathrm{OEt}_{2}$ as a Lewis acid, (4S,5R)-2-acetoxy-5-benzyloxy-4methyltetrahydropyran $(0.098 \mathrm{~g}, 0.37 \mathrm{mmol})$ afforded the product $(0.074 \mathrm{~g}, 81 \%)$ as a 95:5 1,4cis:trans mixture of isomers. The oil was purified by flash chromatography (1:9 EtOAc/hexanes) as eluent: ${ }^{1} \mathrm{H}$ NMR $\left(500 \mathrm{MHz}, \mathrm{C}_{6} \mathrm{D}_{6}\right) \delta 7.30(\mathrm{~d}, J=7.6,2 \mathrm{H}), 7.18-7.08(\mathrm{~m}$, 3H), 5.94-5.86 (m, 1H), $5.03(\mathrm{~m}, 2 \mathrm{H}), 4.39(\mathrm{~d}, J=12.1,1 \mathrm{H}), 4.25(\mathrm{~d}, J=12.1,1 \mathrm{H}), 3.77(\mathrm{dd}, J$ $=12.4,3.8,1 \mathrm{H}), 3.53(\mathrm{~m}, 1 \mathrm{H}), 3.46(\mathrm{dd}, J=12.4,2.3,1 \mathrm{H}), 2.76(\mathrm{~m}, 1 \mathrm{H}), 2.40(\mathrm{~m}, 1 \mathrm{H}), 2.08(\mathrm{~m}$, 1H), $1.97(\mathrm{~m}, 1 \mathrm{H}), 1.85(\mathrm{ddd}, J=13.3,9.1,4.9,1 \mathrm{H}), 1.11(\mathrm{ddd}, J=13.3,4.6,3.2,1 \mathrm{H}), 0.80(\mathrm{~d}, J$ $=7.2,3 \mathrm{H}) ;{ }^{13} \mathrm{C} \mathrm{NMR}\left(125 \mathrm{MHz}, \mathrm{CDCl}_{3}\right) \delta 138.8,135.2,128.3,127.6,127.5,116.7,77.0,71.6$, 70.7, 64.4, 39.2, 33.5, 29.9, 17.3; IR (thin film) 3066, 2928, 1642, 1454, 1097, $698 \mathrm{~cm}^{-1}$; HRMS 
(CI/isobutane) $m / z$ calcd for $\mathrm{C}_{13} \mathrm{H}_{17} \mathrm{O}_{2}\left(\mathrm{M}-\mathrm{C}_{3} \mathrm{H}_{5}\right)^{+}$205.1229, found 205.1219. Anal. Calcd. for $\mathrm{C}_{16} \mathrm{H}_{22} \mathrm{O}_{2}$ : C, 78.01; H, 9.00. Found: C, 78.05; H, 9.06.

\section{Nucleophilic Addition of Diethylzinc}

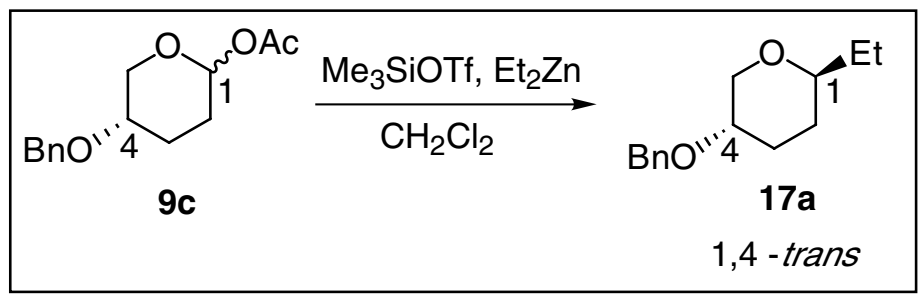

4-Benzyloxy-1-ethyltetrahydropyran (17a): ${ }^{15}$ A solution of 4-benzyloxytetrahydropyran-1acetate $(0.212 \mathrm{~g}, 0.85 \mathrm{mmol})$ in $\mathrm{CH}_{2} \mathrm{Cl}_{2}(3.36 \mathrm{~mL})$ was cooled to $-78{ }^{\circ} \mathrm{C}$. Diethyl zinc $(0.209 \mathrm{~g}$, $1.69 \mathrm{mmol})$ was added followed by TMSOTf $(0.226 \mathrm{~g}, 1.01 \mathrm{mmol})$ and the mixture was stirred for $1 \mathrm{~h}$. The reaction mixture was warmed to room temperature and triethylamine $(0.5 \mathrm{~mL})$ was added followed by saturated aqueous $\mathrm{NaHCO}_{3}(1 \mathrm{~mL})$. The mixture was allowed to stir for 30 min, and then it was filtered. The organic layer was washed with ice cold $1 \mathrm{~N} \mathrm{NaHSO}_{4}(5 \mathrm{~mL})$ and saturated aqueous $\mathrm{NaHCO}_{3}(5 \mathrm{~mL})$, dried over $\mathrm{MgSO}_{4}$, and concentrated in vacuo to afford a 98:2 trans:cis mixture of anomers (as determined by GC analysis) as a yellow oil. The oil was purified by flash chromatography (pentane to 1:9 $\mathrm{Et}_{2} \mathrm{O}$ /pentane) to afford the 1,4-trans isomer as a colorless oil $(0.164 \mathrm{~g}, 88 \%):{ }^{1} \mathrm{H}$ NMR $\left(400 \mathrm{MHz}, \mathrm{CDCl}_{3}\right) \delta 7.39-7.28(\mathrm{~m}, 5 \mathrm{H}), 4.62(\mathrm{~d}, J$ $=11.8,1 \mathrm{H}), 4.56(\mathrm{~d}, J=11.9,1 \mathrm{H}), 4.14-4.10(\mathrm{ddd}, J=10.7,4.6,2.3,1 \mathrm{H}), 3.52-3.45(\mathrm{~m}, 1 \mathrm{H})$, $3.21(\mathrm{t}, J=10.6,1 \mathrm{H}$ and $\mathrm{m}, 1 \mathrm{H}), 2.22(\mathrm{~m}, 1 \mathrm{H}), 1.76(\mathrm{~m}, 1 \mathrm{H}), 1.56-1.42(\mathrm{~m}, 3 \mathrm{H}), 1.41-1.28(\mathrm{~m}$, $1 \mathrm{H}), 0.94(\mathrm{t}, J=7.5,3 \mathrm{H}) ;{ }^{13} \mathrm{C} \mathrm{NMR} \delta\left(125 \mathrm{MHz}, \mathrm{CDCl}_{3}\right) 139.1,128.8,128.7,128.0,79.3,73.8$, 71.18, 71.16, 30.6, 30.5, 29.1, 10.6; IR (thin film) 2937, 1454, 1374, 1249, 1098, $1028 \mathrm{~cm}^{-1}$; HRMS (EI/GCMS) $m / z$ calcd for $\mathrm{C}_{14} \mathrm{H}_{24} \mathrm{NO}_{2}\left(\mathrm{M}+\mathrm{NH}_{4}\right)^{+} 238.1807$, found 238.1807. 


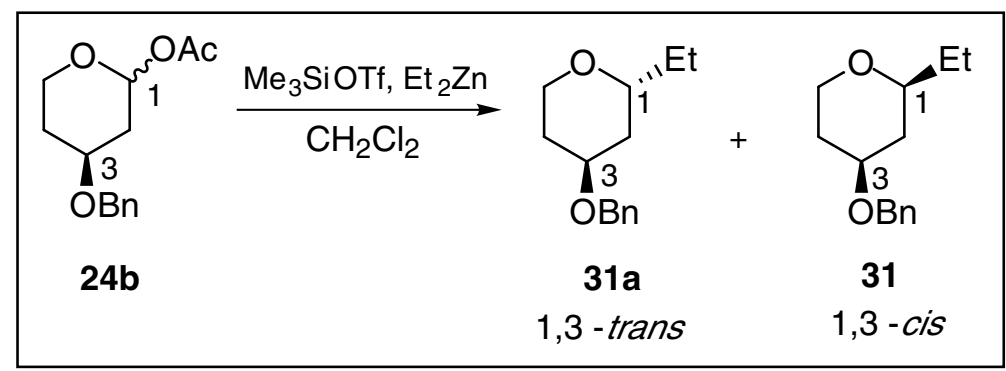

3-Benzyloxy-1-ethyltetrahydropyran (31/31a): ${ }^{15}$ A solution of 3-benzyloxy-tetrahydropyran-1-acetate $(0.039 \mathrm{~g}, 0.157 \mathrm{mmol})$ in $\mathrm{CH}_{2} \mathrm{Cl}_{2}(0.66 \mathrm{~mL})$ was cooled to $-78{ }^{\circ} \mathrm{C}$. Diethyl zinc $(0.286 \mathrm{~mL}, 0.315 \mathrm{mmol})$ was added as a $1.1 \mathrm{M}$ solution in toluene followed by TMSOTf $(0.034$ $\mathrm{mL}, 0.19 \mathrm{mmol}$ ) and the mixture was stirred for $3 \mathrm{~h}$. The reaction mixture was warmed to room temperature and triethylamine $(0.25 \mathrm{~mL})$ followed by saturated aqueous $\mathrm{NaHCO}_{3}(0.5 \mathrm{~mL})$ were added. The mixture was allowed to stir for $30 \mathrm{~min}$ and filtered using $\mathrm{CH}_{2} \mathrm{Cl}_{2}$. The organic layer was washed with ice cold $1 \mathrm{~N} \mathrm{NaHSO}_{4}(2 \mathrm{~mL})$ and saturated aqueous $\mathrm{NaHCO}_{3}(2 \mathrm{~mL})$, dried over $\mathrm{MgSO}_{4}$, and concentrated in vacuo. The reaction afforded a 78:22 cis:trans mixture of anomers, as determined based on GC. The resulting yellow oil was purified by flash column chromatography (pentane to $1: 9 \mathrm{Et}_{2} \mathrm{O} /$ pentane) to afford the product (as a 9:1 cis:trans) as a colorless oil $(\mathrm{g}, \%):{ }^{1} \mathrm{H}$ NMR $\left(400 \mathrm{MHz}, \mathrm{CDCl}_{3}\right) \delta 7.36-7.26(\mathrm{~m}, 5 \mathrm{H}), 7.16(\mathrm{~m}, 5 \mathrm{H}), 4.58(\mathrm{~m}$, $1 \mathrm{H}), 4.53(\mathrm{~m}, 1 \mathrm{H}$ and $\mathrm{m}, 2 \mathrm{H}), 4.05-4.01(\mathrm{ddd}, J=11.8,4.8,1.8,1 \mathrm{H}$ and $\mathrm{m}, 1 \mathrm{H}), 3.91-3.78(\mathrm{~m}$, $2 \mathrm{H}), 3.63(\mathrm{~m}, 1 \mathrm{H}), 3.57-3.50(\mathrm{tt}, J=11.0,4.5,1 \mathrm{H}), 3.40-3.33(\mathrm{td}, J=12.5,2.1,1 \mathrm{H}), 3.20-3.13$ (dddd, $J=11.2,6.7,5.5,1.9,1 \mathrm{H}), 2.08-2.03(\mathrm{ddt}, J=12.4,4.5,2.11 \mathrm{H}), 2.00-1.95(\mathrm{dq}, J=$ 12.4, 2.0, 1H), $1.86(\mathrm{~m}, 1 \mathrm{H}), 1.76(\mathrm{~m}, 1 \mathrm{H}), 1.62-1.37(\mathrm{~m}, 2 \mathrm{H}$ and $\mathrm{m}, 2 \mathrm{H}), 1.29(\mathrm{~m}, 2 \mathrm{H}$ and $\mathrm{m}$, $2 \mathrm{H}), 0.93(\mathrm{t}, J=7.5,3 \mathrm{H}$ and $\mathrm{m}, 3 \mathrm{H}) ;{ }^{13} \mathrm{C} \mathrm{NMR} \delta 128.63,128.59,127.80,127.76,127.67,127.6$, 77.9, 75.0, 73.7, 71.2, 70.2, 69.7, 66.2, 63.1, 38.2, 36.0, 33.0, 30.4, 29.3, 10.1; IR (thin film) 2937, 1454, 1374, 1249, 1098, $1028 \mathrm{~cm}^{-1}$; HRMS (EI/GCMS) $m / z$ calcd for $\mathrm{C}_{14} \mathrm{H}_{20} \mathrm{O}_{2}(\mathrm{M})^{+}$ 220.1462 , found 220.1463 . 


\section{Nucleophilic Addition of 1-Phenyl-1-(trimethylsilyloxy)ethylene}

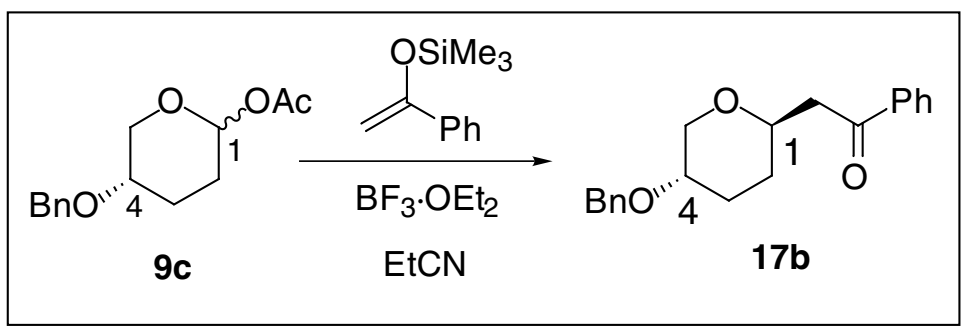

4-Benzyloxy-1-(2-oxo-2-phenylethyl)tetrahydropyran (17b): A solution of 4benzyloxytetrahydropyran-1-acetate $(0.050 \mathrm{~g}, 0.20 \mathrm{mmol})$ in $n$-propionitrile $(2 \mathrm{~mL})$ was cooled to $-78{ }^{\circ} \mathrm{C}$. 1-Phenyl-1-(trimethylsilyloxy)ethylene $(0.041 \mathrm{~mL}, 0.20 \mathrm{mmol})$ was added followed by $\mathrm{BF}_{3} \cdot \mathrm{OEt}_{2}(0.029 \mathrm{~mL}, 0.24 \mathrm{mmol})$ and the mixture was stirred for $5 \mathrm{~min}$. The reaction mixture was quenched with $\mathrm{NaHCO}_{3}(4 \mathrm{~mL})$ and the layers were separated. The aqueous layer was extracted with $\mathrm{CH}_{2} \mathrm{Cl}_{2}(3 \times \mathrm{mL})$, filtered through $\mathrm{Na}_{2} \mathrm{SO}_{4}$, and concentrated in vacuo. The reaction afforded a 96:4 trans:cis mixture of anomers, as determined based on GC. The resulting oil was purified by flash column chromatography (10:90 EtOAc/hexanes) to afford the trans product as a colorless oil $(0.046 \mathrm{~g}, 74 \%):{ }^{1} \mathrm{H}$ NMR $\left(500 \mathrm{MHz}, \mathrm{C}_{6} \mathrm{D}_{6}\right) \delta 7.30(\mathrm{~d}, J=7.6$, 2H), 7.18-7.08 (m, 3H), 5.94-5.86 (m, 1H), 5.03 (m, 2H), 4.39 (d, $J=12.1,1 \mathrm{H}), 4.25$ (d, $J=$ 12.1, 1H), 3.77 (dd, $J=12.4,3.8,1 \mathrm{H}), 3.53(\mathrm{~m}, 1 \mathrm{H}), 3.46(\mathrm{dd}, J=12.4,2.3,1 \mathrm{H}), 2.76(\mathrm{~m}, 1 \mathrm{H})$, $2.40(\mathrm{~m}, 1 \mathrm{H}), 2.08(\mathrm{~m}, 1 \mathrm{H}), 1.97(\mathrm{~m}, 1 \mathrm{H}), 1.85(\mathrm{ddd}, J=13.3,9.1,4.9,1 \mathrm{H}), 1.11$ (ddd, $J=13.3$, 4.6, 3.2, 1H), $0.80(\mathrm{~d}, J=7.2,3 \mathrm{H}) ;{ }^{13} \mathrm{C} \mathrm{NMR}\left(125 \mathrm{MHz}, \mathrm{CDCl}_{3}\right) \delta 198.0,138.5,133.2,128.6$, 127.7, 127.6, 77.3, 74.0, 72.8, 70.8, 70.7, 44.5, 30.6, 30.0; IR (thin film) 2953, 2854, 1684, 1202, 1092, $753 \mathrm{~cm}^{-1}$; HRMS (CI/isobutane) $\mathrm{m} / z$ calcd for $\mathrm{C}_{11} \mathrm{H}_{16} \mathrm{OSi}(\mathrm{M})^{+} 310.1568$, found 310.1562. Anal. Calcd. for $\mathrm{C}_{11} \mathrm{H}_{16} \mathrm{OSi}$ C, 77.39; H, 7.14. Found: C, 77.12; H, 7.20. 


\section{Stereochemical Proofs}

\section{VIA. ${ }^{1}$ H NMR Coupling Constant Data}

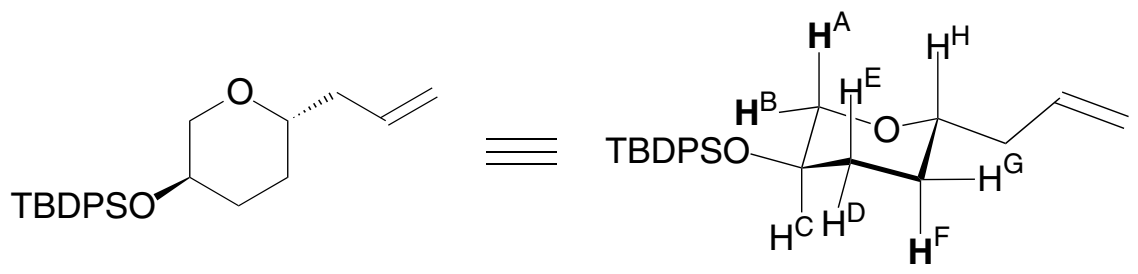

trans-1-Allyl-4-(tert-butyldiphenylsilyloxy)tetrahydropyran (16a)

$\mathbf{H}^{\mathrm{A}}: \mathrm{t}, J=10.3\left(\mathrm{gem}, \mathrm{ax}^{\mathrm{A}}-\mathrm{ax}^{\mathrm{C}}\right)$

$\mathbf{H}^{\mathrm{B}}: \operatorname{ddd}, J=10.7(\mathrm{gem}), 4.9\left(\mathrm{eq}^{\mathrm{B}}-\mathrm{ax}^{\mathrm{C}}\right), 2.3\left(\mathrm{~W}\right.$-coupling to $\left.\mathrm{H}^{\mathrm{D}}\right)$

$\mathbf{H}^{\mathrm{F}}$ : tdd, $J=13.6\left(\mathrm{gem}, \mathrm{ax}^{\mathrm{F}}-\mathrm{ax}^{\mathrm{H} / \mathrm{E}}\right), 11.1\left(\mathrm{ax}^{\mathrm{F}}-\mathrm{ax}^{\mathrm{H} / \mathrm{E}}\right), 3.8\left(\mathrm{ax}^{\mathrm{F}}-\mathrm{eq}^{\mathrm{D}}\right)$<smiles>C=CC[C@H]1CCC(OC)CO1</smiles>

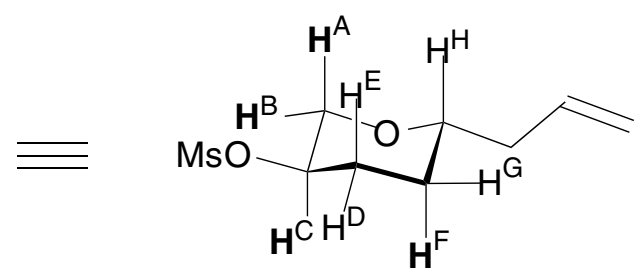

trans-Allyl-4-methanesulfoxytetrahydropyran (16b)

$\mathbf{H}^{\mathrm{A}}: \mathrm{t}, J=10.5\left(\mathrm{gem}, \mathrm{ax}^{\mathrm{A}}-\mathrm{ax}^{\mathrm{C}}\right)$

$\mathbf{H}^{\mathrm{B}}$ : ddd, $J=10.7\left(\right.$ gem), $4.5\left(\mathrm{eq}^{\mathrm{B}}-\mathrm{ax}^{\mathrm{C}}\right), 2.3\left(\mathrm{~W}\right.$-coupling to $\left.\mathrm{H}^{\mathrm{D}}\right)$

$\mathbf{H}^{\mathrm{C}}: \mathrm{tt}, J=10.3\left(\mathrm{ax}^{\mathrm{C}}-\mathrm{ax}^{\mathrm{A}}, \mathrm{ax}^{\mathrm{C}}-\mathrm{ax}^{\mathrm{E}}\right), 5.2\left(\mathrm{ax}^{\mathrm{C}}-\mathrm{eq}^{\mathrm{B}}, \mathrm{ax}^{\mathrm{C}}-\mathrm{eq}^{\mathrm{D}}\right)$

$\mathbf{H}^{\mathrm{F}}$ : tdd, $J=13.5\left(\mathrm{gem}, \mathrm{ax}^{\mathrm{F}}-\mathrm{ax}^{\mathrm{H} / \mathrm{E}}\right), 11.0\left(\mathrm{ax}^{\mathrm{F}}-\mathrm{ax}^{\mathrm{H} / \mathrm{E}}\right), 3.8\left(\mathrm{ax}^{\mathrm{F}}-\mathrm{eq}^{\mathrm{D}}\right)$<smiles>[R6]O[C@@H]1CC[C@H](CC=C)OC1</smiles>

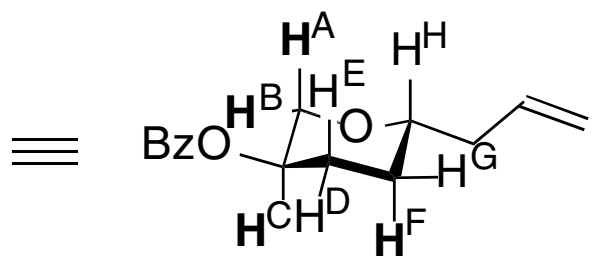

trans-Allyl-4-benzoyloxytetrahydropyran (16c)

$\mathbf{H}^{\mathrm{A}}: \mathrm{t}, J=10.4\left(\mathrm{gem}, \mathrm{ax}^{\mathrm{A}}-\mathrm{ax}^{\mathrm{C}}\right)$

$\mathbf{H}^{\mathrm{B}}$ : ddd, $J=10.6($ gem $), 4.9\left(\mathrm{eq}^{\mathrm{B}}-\mathrm{ax}^{\mathrm{C}}\right), 2.2\left(\mathrm{~W}-\right.$ coupling to $\left.\mathrm{H}^{\mathrm{D}}\right)$

$\mathbf{H}^{\mathrm{C}}: \mathrm{tt}, J=10.5\left(\mathrm{ax}^{\mathrm{C}}-\mathrm{ax}^{\mathrm{A}}, \mathrm{ax}^{\mathrm{C}}-\mathrm{ax}^{\mathrm{E}}\right), 5.4\left(\mathrm{ax}^{\mathrm{C}}-\mathrm{eq}^{\mathrm{B}}, \mathrm{ax}^{\mathrm{C}}-\mathrm{eq}^{\mathrm{D}}\right)$ 


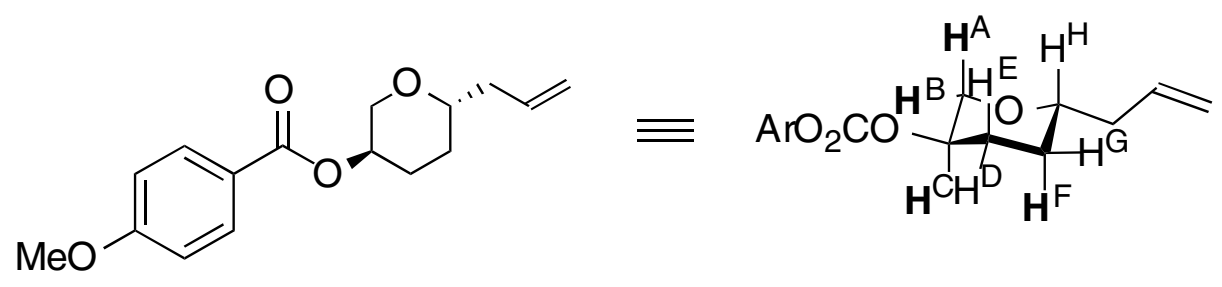

trans-Allyl-4-anisoyloxytetrahydropyran (16d)

$\mathbf{H}^{\mathrm{A}}: \mathrm{t}, J=10.4\left(\mathrm{gem}, \mathrm{ax}^{\mathrm{A}}-\mathrm{ax}^{\mathrm{C}}\right)$

$\mathbf{H}^{\mathrm{B}}$ : ddd, $J=10.5(\mathrm{gem}), 4.9\left(\mathrm{eq}^{\mathrm{B}}-\mathrm{ax}^{\mathrm{C}}\right), 2.1\left(\mathrm{~W}-\right.$ coupling to $\left.\mathrm{H}^{\mathrm{D}}\right)$

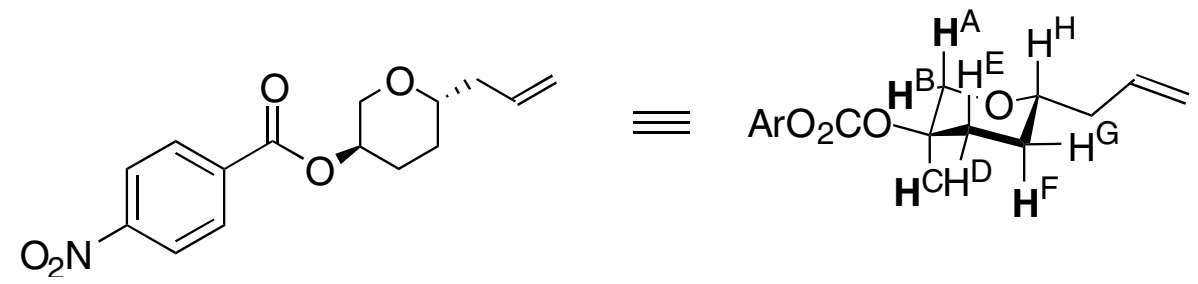

trans-Allyl-4-nitrobenzoyloxytetrahydropyran (16e)

$\mathbf{H}^{\mathrm{A}}: \mathrm{t}, J=10.4\left(\mathrm{gem}, \mathrm{ax}^{\mathrm{A}}-\mathrm{ax}^{\mathrm{C}}\right)$

$\mathbf{H}^{\mathrm{B}}$ : ddd, $J=10.5(\mathrm{gem}), 4.9\left(\mathrm{eq}^{\mathrm{B}}-\mathrm{ax}^{\mathrm{C}}\right), 2.3\left(\mathrm{~W}-\right.$ coupling to $\left.\mathrm{H}^{\mathrm{D}}\right)$<smiles>C=CCC1CCC([N+](=O)[O-])CO1</smiles>
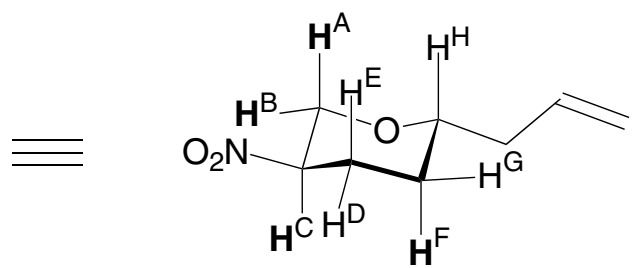

trans-1-Allyl-4-nitrotetrahydropyran (16f)

$\mathbf{H}^{\mathrm{A}}: \mathrm{t}, J=10.7\left(\mathrm{gem}, \mathrm{ax}^{\mathrm{A}}-\mathrm{ax}^{\mathrm{C}}\right)$

$\mathbf{H}^{\mathrm{B}}:$ ddd, $J=10.9($ gem $), 4.6\left(\mathrm{eq}^{\mathrm{B}}-\mathrm{ax}^{\mathrm{C}}\right), 2.4\left(\mathrm{~W}-\right.$ coupling to $\left.\mathrm{H}^{\mathrm{D}}\right)$

$\mathbf{H}^{\mathrm{C}}: \operatorname{ddt}, J=11.9\left(\mathrm{ax}^{\mathrm{C}}-\mathrm{ax}^{\mathrm{A} / \mathrm{E}}\right), 10.5\left(\mathrm{ax}^{\mathrm{C}}-\mathrm{ax}^{\mathrm{A}}\right), 4.5\left(\mathrm{ax}^{\mathrm{C}}-\mathrm{eq}^{\mathrm{B}}, \mathrm{ax}^{\mathrm{C}}-\mathrm{eq}^{\mathrm{D}}\right)$

$\mathbf{H}^{\mathrm{F}}$ : tdd, $J=13.6\left(\mathrm{gem}, \mathrm{ax}^{\mathrm{F}}-\mathrm{ax}^{\mathrm{H} / \mathrm{E}}\right), 11.2\left(\mathrm{ax}^{\mathrm{F}}-\mathrm{ax}^{\mathrm{H} / \mathrm{E}}\right), 4.0\left(\mathrm{ax}^{\mathrm{F}}-\mathrm{eq}^{\mathrm{D}}\right)$ 

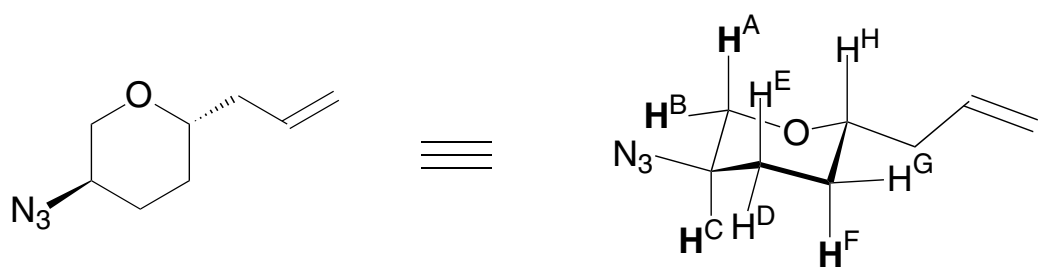

trans-1-Allyl-4-azidotetrahydropyran (16g)

$\mathbf{H}^{\mathrm{A}}: \mathrm{t}, J=10.8\left(\mathrm{gem}, \mathrm{ax}^{\mathrm{A}}-\mathrm{ax}^{\mathrm{C}}\right)$

$\mathbf{H}^{\mathrm{B}}$ : ddd, $J=11.0(\mathrm{gem}), 4.7\left(\mathrm{eq}^{\mathrm{B}}-\mathrm{ax}^{\mathrm{C}}\right), 2.4\left(\mathrm{~W}-\right.$ coupling to $\left.\mathrm{H}^{\mathrm{D}}\right)$

$\mathbf{H}^{\mathrm{C}}: \mathrm{tt}, J=11.0\left(\mathrm{ax}^{\mathrm{C}}-\mathrm{ax}^{\mathrm{A}}, \mathrm{ax}^{\mathrm{C}}-\mathrm{ax}^{\mathrm{E}}\right), 4.7\left(\mathrm{ax}^{\mathrm{C}}-\mathrm{eq}^{\mathrm{B}}, \mathrm{ax}^{\mathrm{C}}-\mathrm{eq}^{\mathrm{D}}\right)$

$\mathbf{H}^{\mathrm{F}}$ : tdd, $J=13.4\left(\mathrm{gem}, \mathrm{ax}^{\mathrm{F}}-\mathrm{ax}^{\mathrm{H} / \mathrm{E}}\right), 10.9\left(\mathrm{ax}^{\mathrm{F}}-\mathrm{ax}^{\mathrm{HE}}\right), 3.7\left(\mathrm{ax}^{\mathrm{F}}-\mathrm{eq}^{\mathrm{D}}\right)$
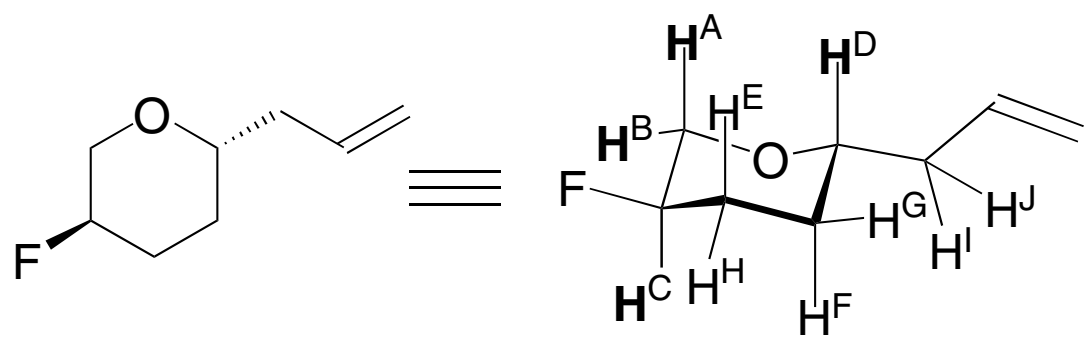

\section{trans-1-Allyl-4-Fluorotetrahydropyran (23a, major)}

$\mathbf{H}^{\mathrm{A}}: \quad \mathrm{td}, J=10.1\left(\mathrm{gem}, \mathrm{ax}^{\mathrm{A}}-\mathrm{ax}^{\mathrm{C}}\right), 3.7\left(\mathrm{ax}-\mathrm{F}^{\mathrm{A}}\right)$

$H^{B}$ : $\quad$ ddt, $J=10.7$ (gem), $5.1\left(\right.$ eq $\left.^{B}-a x^{C}\right), 2.5\left(a x^{B}-F^{e q}, W\right.$-coupling to $\left.H^{D}\right)$

$\mathrm{H}^{\mathrm{C}}$ : $\quad \mathrm{dtt}, J=48.8\left(\mathrm{ax}^{\mathrm{C}}-\mathrm{F}^{\mathrm{eq}}\right), 10.3\left(\mathrm{ax}^{\mathrm{C}}-\mathrm{ax}^{\mathrm{A}}, \mathrm{ax}^{\mathrm{C}}-\mathrm{ax}^{\mathrm{E}}\right), 5.3\left(\mathrm{ax}^{\mathrm{C}}-\mathrm{eq}^{\mathrm{B}}, \mathrm{ax}^{\mathrm{C}}-\mathrm{eq}^{\mathrm{H}}\right)$

$H^{\mathrm{B}}: \quad \mathrm{dtd}, J=10.5\left(a x^{\mathrm{D}}-\mathrm{ax}^{\mathrm{F}}\right), 6.0\left(\mathrm{ax}^{\mathrm{D}}-\mathrm{ax}^{\prime}, \mathrm{ax}^{\mathrm{D}}-\mathrm{ax}{ }^{\mathrm{J}}\right), 21.8\left(\mathrm{ax}^{\mathrm{D}}-\mathrm{eq}^{\mathrm{G}}\right)$<smiles>OCCC[C@H]1CCC(F)CO1</smiles><smiles>OCCCC1CC2(P)OC1(P)C(F)(P)C2F</smiles>

\section{trans 1-(3-Hydroxypropyl)-4-fluorotetrahydropyran (S40)}

$H^{\mathrm{A}}: \quad \mathrm{td}, J=10.4\left(\mathrm{gem}, \mathrm{ax}^{\mathrm{A}}-\mathrm{ax}^{\mathrm{B}}\right)$, $3.3\left(\mathrm{ax}^{\mathrm{A}}-\mathrm{F}^{\mathrm{eq}}\right)$

$\mathbf{H}^{\mathrm{B}}: \quad \mathrm{dtt}, \mathcal{J}=48.7\left(a x^{\mathrm{B}}-\mathrm{F}^{\mathrm{eq}}\right), 10.4\left(a x^{\mathrm{B}}-\mathrm{ax}^{\mathrm{A}}, \mathrm{ax}^{\mathrm{B}}-\mathrm{ax}^{\mathrm{E}}\right), 5.3\left(\mathrm{ax}^{\mathrm{B}}-\mathrm{eq}^{\mathrm{D}}, \mathrm{ax}^{\mathrm{B}}-\mathrm{eq}^{\mathrm{C}}\right)$ 
<smiles>O=C(Nc1ccc([N+](=O)[O-])cc1)OCCC[C@H]1CCC(F)CO1</smiles>

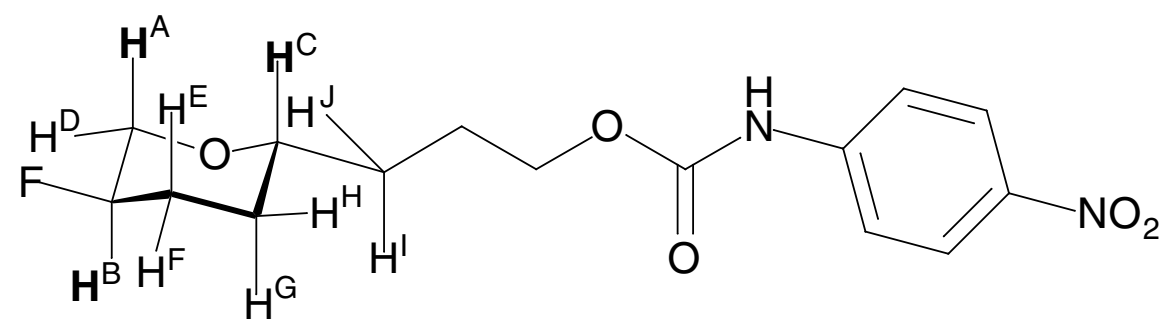

trans-(4-Nitrophenyl)carbamic acid-4-Fluorotetrahydropyran (trans-S41, major) $\mathbf{H}^{\mathrm{A}}: \quad \mathrm{td}, J=10.4\left(\mathrm{gem}, \mathrm{ax} \mathrm{x}^{\mathrm{A}}-\mathrm{ax}^{\mathrm{B}}\right), 3.4\left(\mathrm{ax}^{\mathrm{A}}-\mathrm{F}^{\mathrm{eq}}\right)$

$\mathbf{H}^{\mathrm{B}}: \quad \mathrm{dtt}, J=48.7\left(\mathrm{ax}^{\mathrm{B}}-\mathrm{F}^{\mathrm{eq}}\right), 10.4\left(\mathrm{ax}^{\mathrm{B}}-\mathrm{ax}^{\mathrm{A}}, \mathrm{ax}^{\mathrm{B}}-\mathrm{ax}^{\mathrm{E}}\right), 5.2\left(\mathrm{ax}^{\mathrm{B}}-\mathrm{eq}^{\mathrm{D}}, \mathrm{ax}^{\mathrm{B}}-\mathrm{eq}^{\mathrm{F}}\right)$ $\mathbf{H}^{\mathrm{C}}$ : dddd, $J=10.8\left(\mathrm{ax}^{\mathrm{C}}-\mathrm{H}^{\mathrm{G}}\right), 8.7\left(\mathrm{ax}^{\mathrm{C}}-\mathrm{ax}\right), 3.9\left(\mathrm{ax}^{\mathrm{C}}-\mathrm{eq}^{\mathrm{J}}\right), 2.3\left(\mathrm{ax}^{\mathrm{C}}-\mathrm{eq}^{\mathrm{H}}\right)$<smiles>[B]C1([B])O[C@@H](CC=C)C[C@H](C#C)C1([B])[B]</smiles>

cis-1-Allyl-4-Chlorotetrahydropyran (cis-22b, minor)

$\mathbf{H}^{\mathrm{A}}$ : $\quad \mathrm{dd}, \mathcal{J}=12.7(\mathrm{gem}), 1.8\left(\mathrm{ax}^{\mathrm{A}}-\mathrm{eq}^{\mathrm{C}}\right)$

$H^{B}: \quad d t, J=12.7($ gem $), 1.8\left(e^{B}-e q^{C}, W-\right.$ coupling to $\left.H^{D}\right)$ 


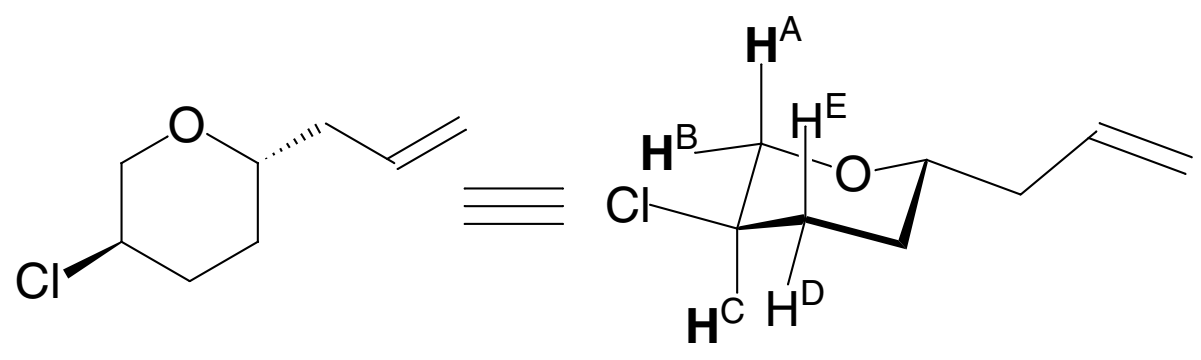

trans-1-Allyl-4-Chlorotetrahydropyran (trans-23b, major) $H^{A}: \quad t, J=10.8\left(\right.$ gem, $\left.a x^{A}-a x^{C}\right)$

$\mathbf{H}^{\mathrm{B}}$ : $\quad$ ddd, $J=10.9($ gem $), 4.7\left(\mathrm{eq}^{\mathrm{B}}-\mathrm{ax}{ }^{\mathrm{C}}\right), 2.3\left(\mathrm{~W}\right.$-coupling to $\left.\mathrm{H}^{\mathrm{D}}\right)$ $\mathbf{H}^{\mathrm{C}}: \quad \mathrm{tt}, J=11.2\left(a x^{\mathrm{C}}-a x^{\mathrm{A}}, \mathrm{ax}^{\mathrm{C}}-a x^{\mathrm{E}}\right), 4.6\left(\mathrm{ax}^{\mathrm{C}}-\mathrm{eq}^{\mathrm{B}}, \mathrm{ax}^{\mathrm{C}}-\mathrm{eq}^{\mathrm{D}}\right)$<smiles>C=CC[C@H]1CCC(Br)CO1</smiles>
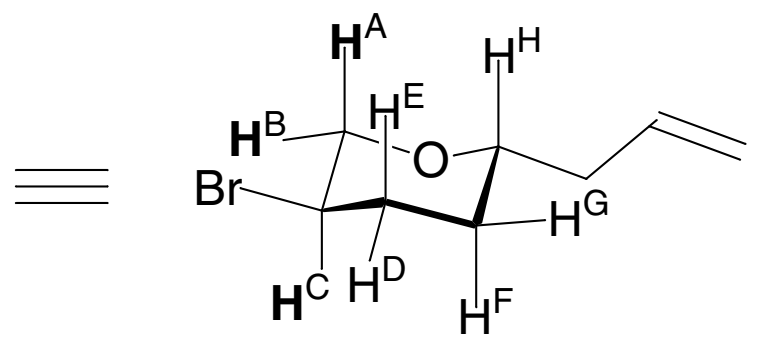

1-Allyl-4-bromotetrahydropyran (23c, major isomer)

$\mathbf{H}^{\mathrm{A}}: \mathrm{t}, J=11.0\left(\mathrm{gem}, \mathrm{ax}^{\mathrm{A}}-\mathrm{ax}^{\mathrm{C}}\right)$

$\mathbf{H}^{\mathrm{B}}:$ ddd, $J=11.0($ gem $), 4.6\left(\mathrm{eq}^{\mathrm{B}}-\mathrm{ax}^{\mathrm{C}}\right), 2.3\left(\mathrm{~W}\right.$-coupling to $\left.\mathrm{H}^{\mathrm{D}}\right)$

$\mathbf{H}^{\mathrm{C}}: \mathrm{tt}, J=11.4\left(\mathrm{ax}^{\mathrm{C}}-\mathrm{ax}^{\mathrm{A}}, \mathrm{ax}^{\mathrm{C}}-\mathrm{ax}^{\mathrm{E}}\right), 4.6\left(\mathrm{ax}^{\mathrm{C}}-\mathrm{eq}^{\mathrm{B}}, \mathrm{ax}^{\mathrm{C}}-\mathrm{eq}^{\mathrm{D}}\right)$

$\mathbf{H}^{\mathrm{F}}$ : tdd, $J=13.4\left(\mathrm{gem}, \mathrm{ax}^{\mathrm{F}}-\mathrm{ax}^{\mathrm{H} / \mathrm{E}}\right), 11.1\left(\mathrm{ax}^{\mathrm{F}}-\mathrm{ax}^{\mathrm{H} / \mathrm{E}}\right), 3.8\left(\mathrm{ax}^{\mathrm{F}}-\mathrm{eq}^{\mathrm{D}}\right)$<smiles>[CH]C1CCC(CC=C)OC1</smiles><smiles>C1#CCC#C1</smiles>

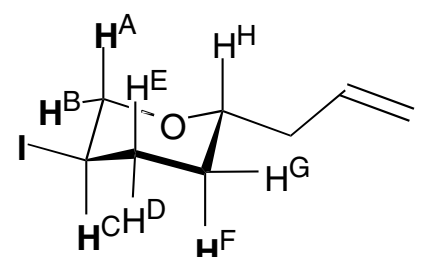

trans-1-Allyl-4-iodotetrahydropyran (23d, the minor isomer)

$\mathbf{H}^{\mathrm{A}}: \mathrm{dd}, J=11.9(\mathrm{gem}), 10.5\left(\mathrm{ax}^{\mathrm{A}}-\mathrm{ax}^{\mathrm{C}}\right)$ 

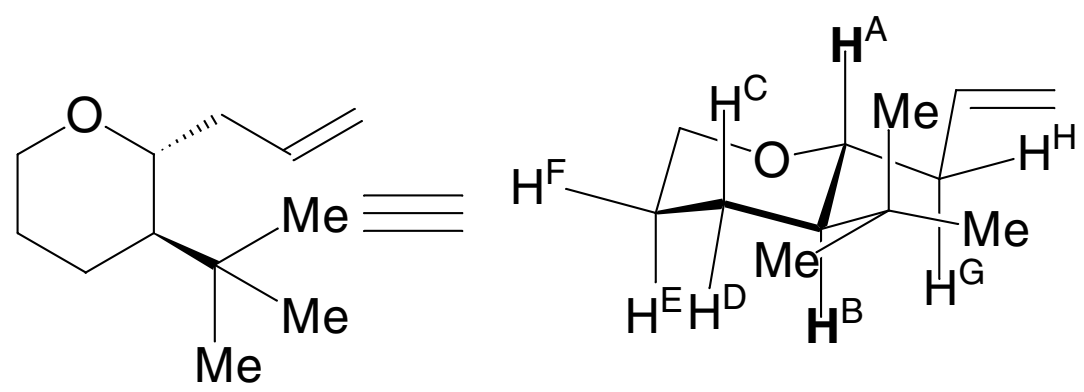

\section{trans-1-Allyl-2-tert-butyltetrahydropyran (34d, major)} $\mathbf{H}^{\mathrm{A}}: \quad \mathrm{ddd}, \mathcal{J}=8.7\left(\mathrm{ax}^{\mathrm{A}}-\mathrm{ax}^{\mathrm{B}}\right), 7.4\left(\mathrm{ax}^{\mathrm{A}}-\mathrm{ax}^{\mathrm{G}}\right), 4.0\left(\mathrm{ax}^{\mathrm{A}}-\mathrm{eq}^{\mathrm{H}}\right)$

$H^{B}: \quad \operatorname{ddd}, J=10.3\left(a x^{B}-a x^{C}\right), 8.6\left(a x^{B}-a x^{A}\right), 4.2\left(a x^{B}-e q^{D}\right)$ $H^{C}:$ tdd, $J=11.1\left(\right.$ gem, $\left.a x^{C}-a x^{B}\right), 8.6\left(a x^{C}-a x^{E}\right), 4.2\left(a x^{C}-e q^{F}\right)$<smiles>C=CC[C@H]1C[C@H](C)[C@H](OCc2ccccc2)CO1</smiles>
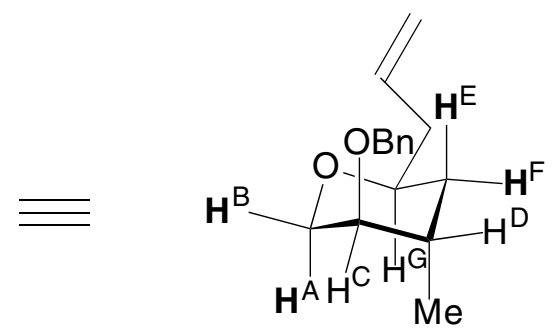

\section{Disubstituted System (44)}

$\mathbf{H}^{\mathrm{A}}: \mathrm{dd}, J=12.4(\mathrm{gem}), 2.3\left(\mathrm{ax}^{\mathrm{A}}-\mathrm{eq}^{\mathrm{C}}\right)$

$\mathbf{H}^{\mathrm{B}}: \mathrm{dd}, J=12.4($ gem $), 3.8\left(\mathrm{eq}^{\mathrm{B}}-\mathrm{eq}^{\mathrm{C}}\right)$

$\mathbf{H}^{\mathrm{E}}:$ ddd, $J=13.3($ gem $), 9.1\left(\mathrm{ax}^{\mathrm{E}}-\mathrm{ax}^{\mathrm{G}}\right), 4.9\left(\mathrm{ax}^{\mathrm{E}}-\mathrm{eq}^{\mathrm{D}}\right)$

$\mathbf{H}^{\mathrm{F}}:$ ddd, $J=13.3($ gem $), 4.6\left(\mathrm{eq}^{\mathrm{F}}-\mathrm{eq}^{\mathrm{D}} / \mathrm{ax}^{\mathrm{G}}\right), 3.2\left(\mathrm{eq}^{\mathrm{F}}-\mathrm{eq}^{\mathrm{D}} / \mathrm{ax}^{\mathrm{G}}\right)$

\section{VIB. DPFGSE-NOE Data}

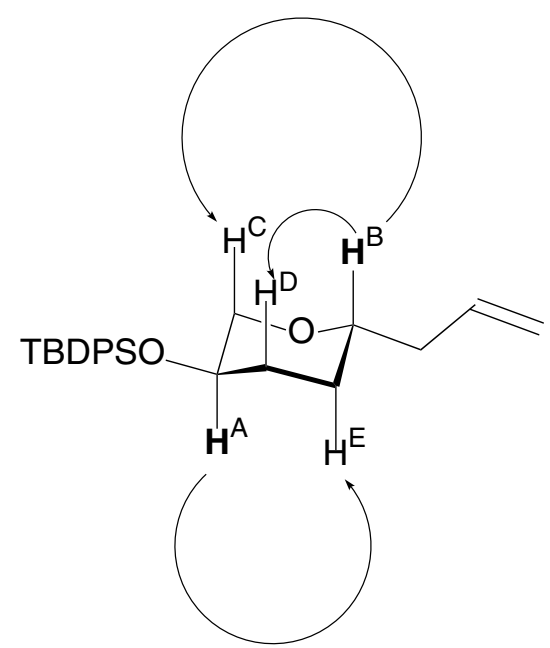

trans-1-Allyl-4-(tert-butyldiphenylsilyloxy)tetrahydropyran (16a) 
$\mathbf{H}^{\mathrm{A}}$ irradiated: $\mathrm{H}^{\mathrm{E}}(4.13 \%)$

$\mathbf{H}^{\mathrm{B}}$ irradiated: $\mathrm{H}^{\mathrm{C}}(8.33 \%), \mathrm{H}^{\mathrm{D}}(3.80 \%)$

Mixing time was $2.0 \mathrm{~s}$.

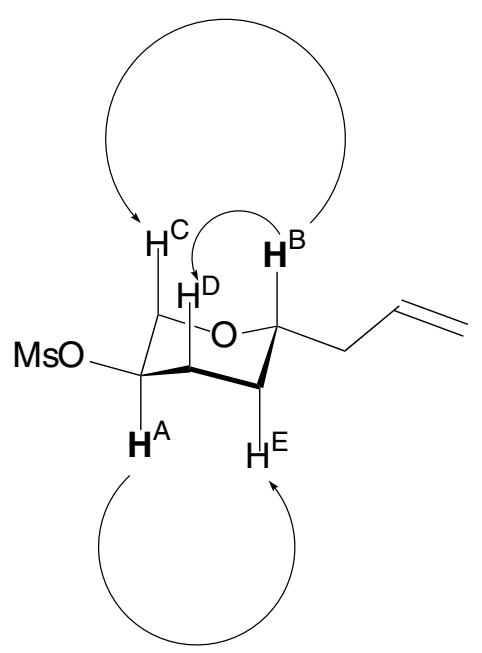

trans-1-Allyl-4-methanesulfoxytetrahydropyran (16b)

$\mathbf{H}^{\mathrm{A}}$ irradiated: $\mathrm{H}^{\mathrm{E}}(2.18 \%)$

$\mathbf{H}^{\mathrm{B}}$ irradiated: $\mathrm{H}^{\mathrm{C}}(4.45 \%), \mathrm{H}^{\mathrm{D}}(2.40 \%)$

Mixing time was $2.0 \mathrm{~s}$.

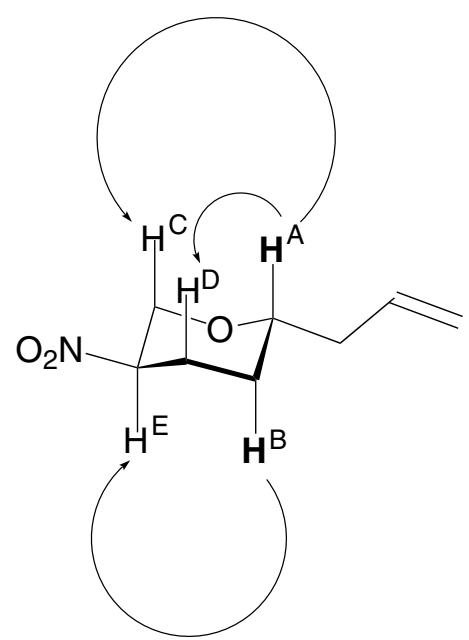

trans-1-Allyl-4-Nitrotetrahydropyran (16f)

$\mathbf{H}^{\mathrm{A}}$ irradiated: $\mathrm{H}^{\mathrm{C}}(3.85 \%), \mathrm{H}^{\mathrm{D}}(1.94 \%)$

$\mathbf{H}^{\mathrm{B}}$ irradiated: $\mathrm{H}^{\mathrm{E}}(2.80 \%)$

Mixing time was $2.0 \mathrm{~s}$. 


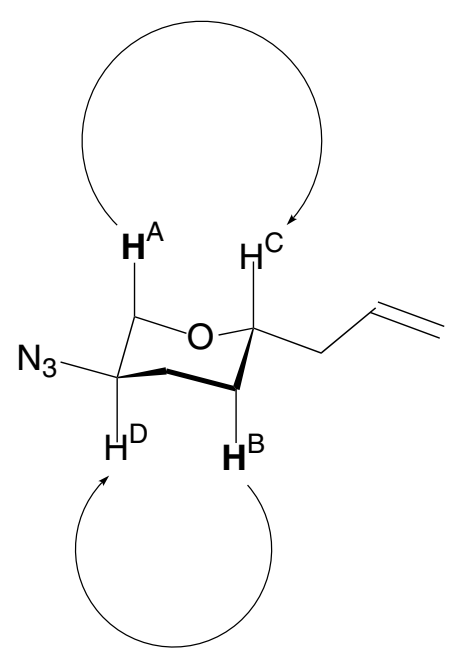

trans-1-Allyl-4-azidotetrahydropyran (16g)

$\mathbf{H}^{\mathrm{A}}$ irradiated: $\mathrm{H}^{\mathrm{C}}(3.36 \%)$

$\mathbf{H}^{\mathrm{B}}$ irradiated: $\mathrm{H}^{\mathrm{D}}(2.34 \%)$

Mixing time was $2.0 \mathrm{~s}$.

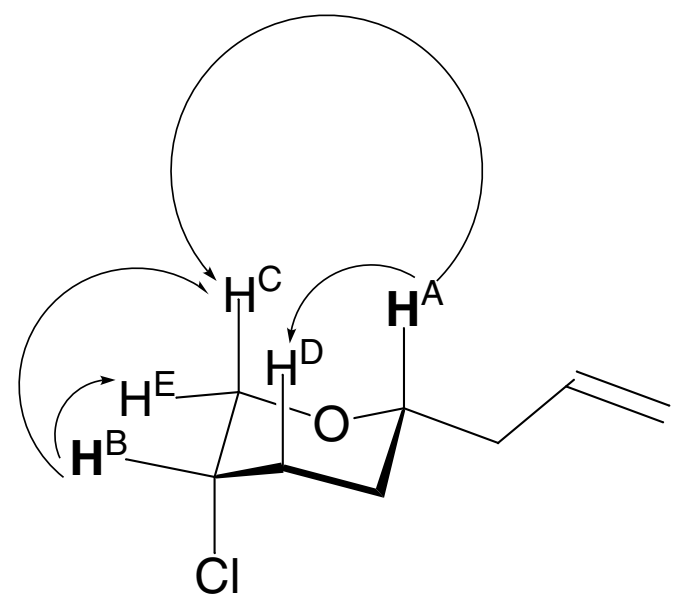

cis-1-Allyl-4-Chlorotetrahydropyran (cis-22b, minor)

$H^{\mathrm{A}}$ irradiated: $\mathrm{H}^{\mathrm{C}}(2.90 \%), \mathrm{H}^{\mathrm{D}}(1.72 \%)$

$H^{\mathrm{B}}$ irradiated: $\mathrm{H}^{\mathrm{C}}(2.21 \%), \mathrm{H}^{\mathrm{E}}(0.40 \%)$

(Mixing time was $2.0 \mathrm{~s}$.) 


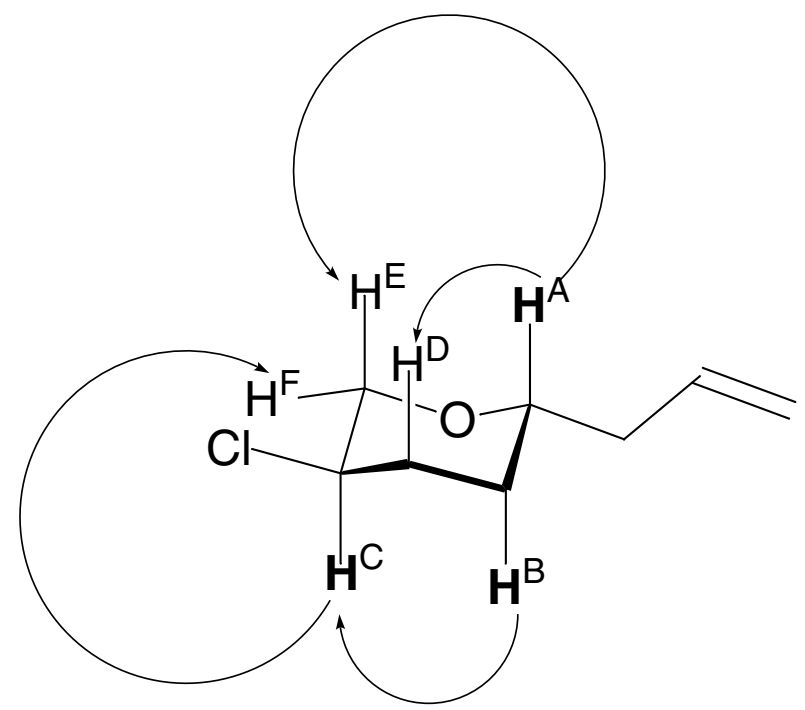

trans-1-Allyl-4-Chlorotetrahydropyran (trans-23b, major)

$H^{A}$ irradiated: $H^{D}(1.97 \%), H^{E}(2.77 \%)$

$H^{\mathrm{B}}$ irradiated: $\mathrm{H}^{\mathrm{C}}(1.71 \%)$

$\mathrm{H}^{\mathrm{C}}$ irradiated: $\mathrm{H}^{\mathrm{F}}(0.92 \%)$

Note: There was no NOE observed between $\mathrm{H}^{\mathrm{C}}$ and $\mathrm{H}^{\mathrm{E}}$. (Mixing time was $2.0 \mathrm{~s}$.)

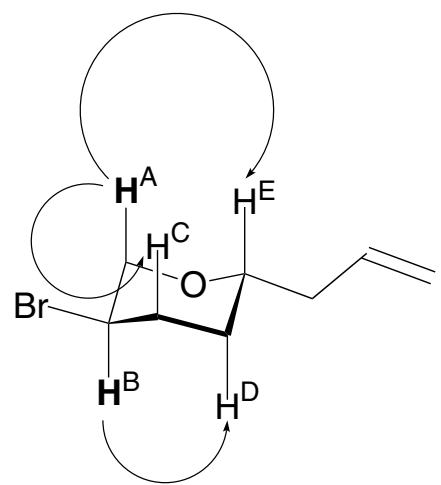

1-Allyl-4-bromotetrahydropyran (23c, major isomer)

$\mathbf{H}^{\mathrm{A}}$ irradiated: $\mathrm{H}^{\mathrm{C}}(1.20 \%), \mathrm{H}^{\mathrm{E}}(3.24 \%)$

$\mathbf{H}^{\mathrm{B}}$ irradiated: $\mathrm{H}^{\mathrm{D}}(1.46 \%)$

Mixing time was $2.0 \mathrm{~s}$. 


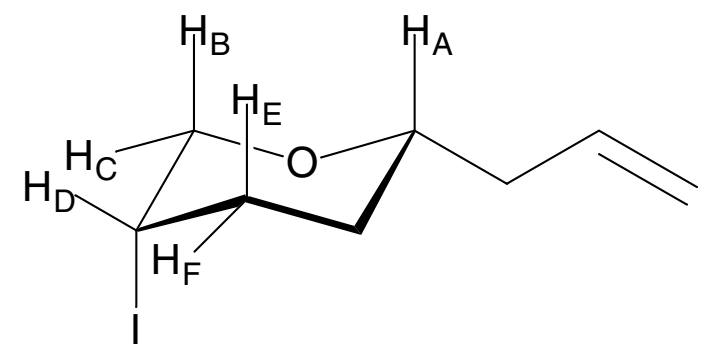

\section{Cis-1-allyl-4-iodotetrahydropyran (22d, major isomer)}

$\mathbf{H}^{\mathrm{A}}$ irradiated: $\mathrm{H}^{\mathrm{B}}(2.57 \%), \mathrm{H}^{\mathrm{E}}(1.61 \%)$

$\mathbf{H}^{\mathbf{B}}$ irradiated: $\mathrm{H}^{\mathrm{C}}(4.55 \%), \mathrm{H}^{\mathrm{D}}(0.77 \%)$

$\mathbf{H}^{\mathrm{C}}$ irradiated: $\mathrm{H}^{\mathrm{B}}(1.05 \%), \mathrm{H}^{\mathrm{D}}(1.65 \%)$

$\mathbf{H}^{\mathrm{D}}$ irradiated: $\mathrm{H}^{\mathrm{C}}(1.10 \%), \mathrm{H}^{\mathrm{B}}(2.20 \%)$

$\mathbf{H}^{\mathbf{F}}$ irradiated: $\mathrm{H}^{\mathrm{D}}(1.41 \%)$

(Mixing time was two seconds.)

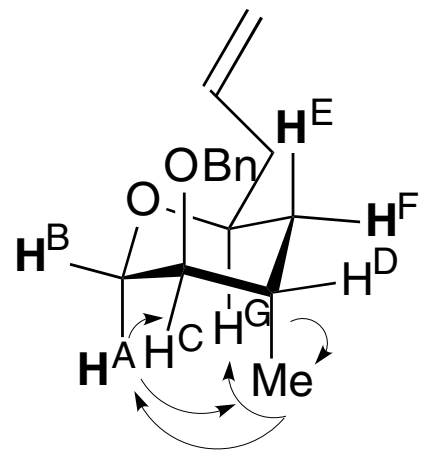

\section{Disubstituted Compound (44)}

Me irradiated: $\mathrm{H}^{\mathrm{A}}(0.4 \%), \mathrm{H}^{\mathrm{G}}(0.5 \%)$

$\mathbf{H}^{\mathbf{G}}$ irradiated: $\mathrm{Me}(1.4 \%)$

$\mathbf{H}^{\mathbf{A}}$ irradiated: $\mathrm{Me}(1.3 \%), \mathrm{H}^{\mathrm{c}}(1.7 \%)$

(Mixing time was two seconds.) 


\section{Bibliography}

(1) Pangborn, A. B.; Giardello, M. H.; Grubbs, R. H.; Rosen, R. K.; Timmers, F. J. Organometallics 1996, 15, 1518-1520.

(2) Brown, H. C.; Prasad, J. V. N.; Zee, S. H. J. Am. Chem. Soc. 1985, 50, 1582-1589.

(3) The anomer ratio was determined using ${ }^{1} \mathrm{H}$ NMR spectroscopy

(4) Kaji, E.; Kohno, H.; Zen, S. Bull. Chem. Soc. Jpn 1977, 50, 928-932.

(5) Thoi-Lai, N.; Fraisse-Jullien, R. Bull. Soc. Chim. de France 1967, 10, 3904-3909.

(6) Dess, D. B.; Martin, J. C. J. Am. Chem. Soc. 1991, 113, 7277-7287.

(7) Ireland, R. E.; Liu, L. J. Org. Chem. 1993, 58, 2899.

(8) Hegedus, L. S.; Colson, P.-J. J. Org. Chem. 1994, 59, 4972-4976.

(9) Feldman, K. S.; Wrobleski, M. L. J. Org. Chem. 2000, 65, 3904-3909.

(10) Liu, H.; Sadamoto, R.; Sears, P. S.; Wong, C.-H. J. Am. Chem. Soc. 2001, 123, 9916-9917.

(11) Ho, P.-T.; Davies, N. J. Org. Chem. 1984, 49, 3027-3029.

(12) Clasby, M. C.; Craid, D.; Jaxa-Chamiec, A. A.; Laai, J. Y. Q.; Marsh, A.; Slawin, A. M. Z.; White, A. J. P.; Williams, D. J. Tetrahedron 1996, 52, 4769-4802.

(13) Nicolaou, K. C.; Liu, J. J.; Yang, Z.; Veno, H.; Sorensen, E. J. J. Am. Chem. Soc. 1995, 117, 634.

(14) Roush, W. R.; Ando, K.; Powers, D. B.; Palkowitz, A. D.; Halterman, R. L. J. Am. Chem. Soc. 1990, 112, 6339-6348.

(15) Rychnovsky, S. D.; Powell, N. A. J. Org. Chem. 1999, 64, 2026-2037. 